\title{
The parameter space of galaxy formation
}

\author{
R. G. Bower, ${ }^{\star}{ }^{\star}$ I. Vernon, ${ }^{2}$ M. Goldstein, ${ }^{2}$ A. J. Benson, ${ }^{3}$ C. G. Lacey, ${ }^{1}$ C. M. Baugh, ${ }^{1}$ \\ S. Cole ${ }^{1}$ and C. S. Frenk ${ }^{1}$ \\ ${ }^{1}$ Institute for Computational Cosmology, Department of Physics, University of Durham, South Road, Durham DH1 3LE \\ ${ }^{2}$ Department of Mathematics, University of Durham, South Road, Durham DH1 3LE \\ ${ }^{3}$ Mail Code 350-17, California Institute of Technology, Pasadena, CA 91125, USA
}

Accepted 2010 May 10. Received 2010 April 5; in original form 2009 December 10

\begin{abstract}
Semi-analytic models are a powerful tool for studying the formation of galaxies. However, these models inevitably involve a significant number of poorly constrained parameters that must be adjusted to provide an acceptable match to the observed Universe. In this paper, we set out to quantify the degree to which observational data sets can constrain the model parameters. By revealing degeneracies in the parameter space we can hope to better understand the key physical processes probed by the data. We use novel mathematical techniques to explore the parameter space of the GALFORM semi-analytic model. We base our investigation on the Bower et al. version of GALFORM, adopting the same methodology of selecting model parameters based on an acceptable match to the local $b_{\mathrm{J}}$ and $K$ luminosity functions. Since the GALFORM model is inherently approximate, we explicitly include a model discrepancy term when deciding if a match is acceptable or not. The model contains 16 parameters that are poorly constrained by our prior understanding of the galaxy formation processes and that can plausibly be adjusted between reasonable limits. We investigate this parameter space using the Model Emulator technique, constructing a Bayesian approximation to the GALFORM model that can be rapidly evaluated at any point in parameter space. The emulator returns both an expectation for the GALFORM model and an uncertainty which allows us to eliminate regions of parameter space in which it is implausible that a GALFORM run would match the luminosity function data. By combining successive waves of emulation, we show that only 0.26 per cent of the initial volume is of interest for further exploration. However, within this region we show that the Bower et al. model is only one choice from an extended subspace of model parameters that can provide equally acceptable fits to the luminosity function data. We explore the geometry of this region and begin to explore the physical connections between parameters that are exposed by this analysis. We also consider the impact of adding additional observational data to further constrain the parameter space. We see that the known tensions existing in the Bower et al. model lead to a further reduction in the successful parameter space.
\end{abstract}

Key words: galaxies: formation - galaxies: luminosity function, mass function

\section{INTRODUCTION}

Semi-analytic galaxy formation models are a successful tool for exploring the physical processes responsible for galaxy formation. In essence this technique aims to understand the formation of galaxies by breaking the problem down into a discrete set of (typically non-linear) differential equations describing each physical process. For example, the amount of gas able to cool from the halo depends non-linearly on the halo mass and its gas content. These discrete

${ }^{\star}$ E-mail: r.g.bower@durham.ac.uk processes are then coupled through a set of interactions. For example, the cold gas mass grows as a result of gas accretion and cooling and decreases as a result of star formation and gas ejection. In simple cases, the network of equations can be integrated analytically to make quantitative predictions for the properties of the galaxy population. In more complex cases, the set of equations must be solved numerically, but the computational task is still minor compared to integrating fundamental physical laws on a particle-by-particle (or cell-by-cell) basis, as required by a fully numerical approach (for a few state-of-the-art examples of the fully numerical approach see Crain et al. 2009; Gnedin, Tassis \& Kravtsov 2009; Schaye et al. 2010). However, although the description of each individual 
component of the semi-analytic model may appear simple, the complex interplay between the components means that the outcome of a model is notoriously hard to predict.

Nevertheless, such models have been very successful in defining our current picture of how galaxies form. Initial models, such as Lacey \& Silk (1991), White \& Frenk (1991), Kauffmann, White \& Guiderdoni (1993) and Cole et al. (1994), showed how the formation of galaxies resulted from a competition between gas cooling and accretion, and the ejection of gas from galaxies in supernovadriven winds. This type of feedback explained the observed paucity of faint galaxies compared to the high abundance of low-mass cold dark matter (CDM) haloes. By incorporating these effects into a realistic model for the growth of dark matter haloes and galaxies, these models were able to make a quantitative connection between the assumptions about gas cooling, star formation, feedback, merging and other physical ingredients, and the observed properties of galaxies. Over the past two decades, the sophistication of these models has increased, allowing them to make predictions for many more observational properties such as galaxy sizes, colours, infrared luminosities and correlation functions (e.g. Kauffmann et al. 1999; Somerville \& Primack 1999; Cole et al. 2000; Granato et al. 2000, 2004; Baugh et al. 2005; Menci et al. 2005, 2006; Cattaneo et al. 2006; Kang, Jing \& Silk 2006; Monaco, Fontanot \& Taffoni 2007). At the same time, the improvement in our knowledge of the cosmological parameters has tied down some of the major uncertainties in the input physical description (e.g. Dunkley et al. 2009). As a result, the comparative power of the models has increased.

A particular issue that has been revealed is the need for additional physics to match the sharp break at the bright end of the galaxy luminosity function. A number of additional physical processes have been proposed (cf. Benson et al. 2003a) but the currently favoured explanation centres on an additional feedback channel motivated by observations of the interactions between radio galaxies and the surrounding IGM in clusters. Although implementations differ, the aim of this 'radio-mode' feedback is to suppress cooling in the most massive haloes leading to the sharp break in the luminosity function (Bower et al. 2006, hereafter Bow06; Cattaneo et al. 2006; Croton et al. 2006; Somerville et al. 2008). An important result of implementing this type of feedback in the models is that they then predict that much of the star formation in the largest galaxies will be completed relatively early in the history of the Universe, in many cases above redshift 2 . This has largely eased the conflict between observations of a large population of passive galaxies at high redshift and the tendency for CDM models to form the largest dark matter structures only recently (Bow06).

Despite these successes, the semi-analytic technique has been criticized for a perceived lack of predictive power. Each component of the model must simply encapsulate the physical process that it describes. However, since the processes are poorly understood, this almost inevitably involves parametrizing the process in such a way that our limited knowledge or understanding can be included by allowing parameters to vary between plausible limits. By comparing the model to a limited set of observational results, the model can be calibrated and then, with the values of the parameters fixed, the model can be tested against additional observational constraints. While the traditional approach, such as that used in Cole et al. (2000) or Bow06, is iterate on an initial guess to find a single set of parameters that adequately match the calibration data, this is clearly a Bayesian problem in which we should seek to use the observational data to successively constrain the parameter space of acceptable models.
In this paper we set out to make a systematic exploration of the parameter space of the Bow06 version of the GALFORM model. This contains 16 parameters which can reasonably be adjusted over a plausible range. We note earlier GALFORM models have considered an even larger parameter space: for example, the Baugh et al. (2005) model uses a different parametrization for the disc star formation time-scale, includes a mode of superwind feedback (e.g. Benson et al. 2003a), allows for a different initial mass function (IMF) in starbursts from that in disc star formation and uses different descriptions of gas cooling and gas reheating (cf. Bow06). These differences are not considered here - our purpose is to compare the parameter set identified by Bow06 with the full parameter space available in that model. We explore the effect of introducing additional physical processes in Benson \& Bower (2010).

A variety of strategies for calibrating the model parameters have been adopted in published semi-analytical models. The majority of models have used observational data on selected galaxy properties at $z=0$ to choose a 'best-fitting' set of parameters, and have then made predictions for higher redshifts, but some models have also supplemented the $z=0$ constraints with observational data on high-redshift galaxies when choosing the 'best-fitting' model parameters. Different authors have made different choices as to what is the best set of $z=0$ properties to use in setting the model parameters. For example, Kauffmann et al. (1993, 1999), Somerville \& Primack (1999) and De Lucia, Kauffmann \& White (2004) used the normalization of the Tully-Fisher (TF) relation and the gas masses of Milky Way like galaxies as their primary observational constraints. On the other hand, Cole et al. (1994, 2000), Nagashima et al. (2001), Kang et al. (2006), Baugh et al. (2005) and Bow06 all used the galaxy optical and near-IR luminosity functions as their primary constraints. In addition, most models have used additional $z=0$ properties beyond their 'primary' constraint in choosing best-fitting parameters. For example, Cole et al. (2000) used the gas fractions and sizes of galaxy discs, together with the ratio of early to late morphological types and the stellar metallicities of elliptical galaxies, in addition to the $B$ - and $K$-band luminosity functions. In contrast to this, Benson et al. (2003a) and Bow06 chose to focus on obtaining a good match to the $z=0$ $B$ - and $K$-band luminosity functions [but it is important to note that the starting point for iteration was taken from the Cole et al. (2000) model]. Several subsequent papers have explored the performance of the Bow06 model with respect to additional data sets (e.g. Bower, McCarthy \& Benson 2008; Font et al. 2008; González et al. 2009; Gonzalez-Perez et al. 2009; Kim et al. 2009).

These different strategies for calibrating the model parameters have different advantages and drawbacks. The Bow06 approach has the advantage of simplicity, and that the $z=0$ luminosity functions are measured accurately and (largely) free from observational selection effects. Furthermore, the model outputs do not require a highly complex layer of additional processing to cast them into the observed quantities (of course population synthesis models are still required). A disadvantage is that the present-day optical and nearIR luminosity functions are relatively insensitive to some model parameters, such as those controlling the star formation time-scale (e.g. Cole et al. 2000). For this reason, it is helpful to introduce additional observational constraints to which these other model parameters are more sensitive. For example, Cole et al. (2000) found that the gas mass versus luminosity relation for disc galaxies provides very good constraints on the model parameters for star formation. Potential drawbacks of introducing extra observational constraints beyond the $z=0$ luminosity functions are that they may be less accurately determined observationally, and that a subjective decision 
is required to assign relative weights to the different observational constraints. In addition, if all the available data sets are used to constrain the model, no observations will be immediately available to independently test its validity. This is a deep philosophical issue that we will not tackle here, but clearly we should seek a strategy in which the physical role of each constraint is clear.

Thus, given the wide variety of observational data that could be used to constrain semi-analytical models, each with their own random and systematic errors, what is needed is some more objective procedures for evaluating what is the range of model parameters consistent with a particular combination of observational constraints, and what is the effect on this range of adding or removing a particular observational constraint. In this way, we hope to end up with an objective measure of how robust different predictions from the model are, including how sensitive they are to including different model ingredients and different observational constraints.

This paper is a first step in this program. We introduce a new method of exploring the model parameter space to identify those regions that produce acceptable matches to the observational data. For simplicity, in this paper we follow the approach of Bow06 and use only the $b_{\mathrm{J}}$ and $K$-band $z=0$ luminosity functions to directly constrain the acceptable regions of parameter space. Once we have identified these regions, we briefly examine the performance of the model with respect to additional $z=0$ data sets, but these are not used to define the initial search criterion. Furthermore, we use the same version of GALFORM as in Bow06, making it simple to compare the unique parameter set presented in Bow06 with the full parameter space that we identify in our search here. Since the Bow06 model is implemented on the Millennium $N$-body simulation (Springel et al. 2005), we adopt the same fixed cosmological parameter set. In principle, the methods we present here could be extended to allow the cosmological background model to vary.

Our investigation aims to address some key questions: how large is the range of parameter space that produces acceptable fits? Is the parameter set selected in Bow06 in some sense typical or optimal? It is unlikely that there is a single 'best value'. Given the relatively large number of model parameters, there will be a range of parameter values giving acceptable fits. Moreover, we should be careful to define what we mean by an 'acceptable' fit. Since the GALFORM model is only an approximation to reality, we would not expect the model to exactly reproduce all the observational data, even if the model's parameters were set to their 'best' values. The Bayesian approach we adopt requires us to formalize this uncertainty by introducing a 'model discrepancy' term $\left(\epsilon_{\mathrm{md}}\right)$ into our comparison with the data. This has the effect of ensuring that we do not reject a region of parameter space if the comparison with the data is sufficiently good that future improvements in the model (which reduce the degree of approximation) may result in improved agreement with the data (in that region). This approach is fundamentally different from simply requiring that we find the region of agreement within the observational uncertainties - it recognizes that the model is itself approximate. Ignoring $\epsilon_{\mathrm{md}}$ will lead us to focus on an unjustifiably narrow region of parameter space. In this case, reducing the level of approximation in the model would cause new regions of acceptable parameter space to appear in areas that were previously deemed implausible.

Of course, estimation of $\epsilon_{\mathrm{md}}$ is uncertain. In principle, one could hope to arrive at a value by tracking changes to the model as the level of approximation is reduced. This approach is an active subject in the statistical literature (e.g. Goldstein \& Rougier 2009), but the methods are not yet suitable for application to GALFORM. Instead, we addressed the model discrepancy term by constructing a series of test luminosity functions and asking ourselves whether we would comfortably reject the corresponding region of parameter space on the basis of the comparison and our previous experience of improvements to the GALFORM code. Reassuringly, our estimate of $\epsilon_{\mathrm{md}}$ results in the Bow06 being marginally acceptable. Thus the model discrepancy is consistent with our aim of searching parameter space for parameter sets that perform comparably to (or better than) Bow06.

Our task is therefore to evaluate the GALFORM model over the input parameter space, identifying the portion of this space for which fits to the local luminosity functions are acceptable. Unfortunately, the 16-dimensional (16D) parameter space (that is introduced below) is extremely large. Dividing each axis into (just) five values and exploring all possible parameter combinations would require $10^{11}$ evaluations of the model (and hence require computing time in excess of $10^{8} \mathrm{CPU}$ years). Even if this were possible, the resulting grid would be of such low resolution that it would give little indication of the GALFORM parameter space. Clearly a much better targeted strategy can be devised.

In this paper, we use the 'model emulator' technique (e.g. Craig et al. 1997; Kennedy \& O’Hagan 2001; Vernon, Goldstein \& Bower 2010) to explore the parameter space. This technique has been specifically developed within the statistics community in order to analyse models that possess high-dimensional parameter spaces. It involves constructing a stochastic model that emulates the output of the GALFORM model. The emulator is constructed so as to reproduce the results of known runs and statistically interpolate between them taking into account the appropriate correlation length of the model. At each new point, the model provides an expectation value for the outcome of a GALFORM evaluation and a variance reflecting the degree of uncertainty in the emulator output. An evaluation of the emulator is of the order of $10^{7}$ times faster than an evaluation of the full model, and the emulator can therefore be used to eliminate regions of parameter space for which it is implausible that an evaluation of GALFORM will result in an acceptable match to the observational data. By proceeding in waves of emulation, we successively reduce the volume that must be investigated at each level until the volume that must be directly evaluated is a tiny fraction (less than 0.3 per cent) of the original parameter space. The primary advantage of the emulator is its speed, which allows us to investigate the full parameter space efficiently and restricts time-consuming evaluations of the GALFORM model to regions of parameter space where the outcome cannot be predicted with sufficient accuracy by the emulator. Combining the emulator method with an efficient strategy for sparsely sampling the parameter space, we can explore the parameter space of galaxy formation with around a month of CPU time. These techniques are gaining widespread acceptance in the climate research community where full evaluations of the computer model are prohibitively expensive. The parallel with the galaxy formation problem is powerful and illustrative (Vernon et al. 2010). Our work is also closely related to studies of the galaxy formation parameter space that are based on exploration with Monte Carlo Markov chain (MCMC) techniques (Kampakoglou, Trotta \& Silk 2008; Henriques et al. 2009). These methods currently consider lower dimensionality than we address here, and it should be noted that, in general, MCMC techniques may face problems when dealing with high-dimensional input spaces. We should also stress that the dimensionality of the problem that we consider here is likely to greatly increase as additional physical processes are included in the model. Indeed, this has been one of the major motivations for the development of the emulation techniques presented here (Oakley \& O'Hagan 2004). For a summary of state-of-the-art emulation 
techniques see the Managing Uncertainty for Complex Models web site http://mucm.group.shef.ac.uk/index.html.

The emulator process identifies a small fraction of the total input space as generating acceptable luminosity functions. The geometry and extent of the region is, however, hard to comprehend. As we will see, some parameters (or parameter combinations) are poorly constrained: this can be viewed as telling us that these have little role in determining certain observable properties of galaxies. Conversely, some parameter combinations are tightly constrained: these play a critical role, and we can hope to use this to understand more about the interplay of the components in the GALFORM model, and thus to better understand the physics underlying the galaxy formation process.

As we have already stressed, this paper concentrates on the Bow06 version of the GALFORM code. Future papers will explore the much larger parameter space created by recent updates to the code, introducing new physical processes to the problem, such as better treatment of angular momentum, a physical description of ram-pressure stripping (cf. Font et al. 2008), AGN heating of halo gas (Bower et al. 2008) and a variable stellar IMF (Baugh et al. 2005). We also extend our new parameter search technique to use a wider range of calibration data from the outset (cf. Benson \& Bower, 2010).

The paper is laid out as follows. In Section 2, we provide a brief overview of the GALFORM code, and outline the physical meaning of the parameters that we vary in this project. In Section 3, we describe the model emulator technique on which our parameter exploration is based. Section 4 presents the main results, Section 4.1 focussing on our success in emulating the luminosity function and its dependence on the model's parameters. Although it is not the primary focus of the paper, it is obviously of interest to see whether additional data sets break the degeneracies evident in the luminosity function comparison. In Section 4.2, we briefly investigate the role of additional data sets. In Section 5, we examine the physical implications of these results using a principal component analysis (PCA) to identify important combinations of the input parameters. Finally, we present a discussion of our work in Section 6 and briefly summarize our main conclusions in Section 7. Throughout, we adopt a cosmology in which $\Omega_{\mathrm{b}}=0.045, \Omega_{\mathrm{M}}=0.25, \Lambda=0.75$ and $\sigma_{8}=0.9$ at the present day. The model assumes $H_{0}=73 \mathrm{~km} \mathrm{~s}^{-1} \mathrm{Mpc}^{-1}$, although we quote luminosities and space densities in term of $h=$ $H_{0} / 100 \mathrm{~km} \mathrm{~s}^{-1} \mathrm{Mpc}^{-1}$.

\section{PARAMETERS OF THE GALFORM CODE}

The GALFORM code contains many parameters. In Table 1, we list the parameters used in the Bow06 version of the code, together with the range of plausible values considered in our analysis. We have grouped the parameters by the physical processes that they are associated with. Below, we briefly describe the parameters. For a full description, we refer the reader to Cole et al. (2000), Baugh et al. (2005) and Bow06.

The first set of parameters is associated with star formation: $\epsilon_{\star}$ determines the normalization of the star formation efficiency, while $\alpha_{\star}$ determines its dependence on the disc circular speed:

$\mathrm{SFR}=\epsilon_{\star}\left(V_{\mathrm{c}, \text { disc }} / 200 \mathrm{~km} \mathrm{~s}^{-1}\right)^{-\alpha_{\star}}\left(M_{\text {cold }} / \tau_{\text {dyn,disc }}\right)$,

where SFR is the star formation rate, $M_{\text {cold }}$ the mass of cold gas in the galaxy, $V_{\mathrm{c}, \text { disc }}$ is the circular velocity of the disc and $\tau_{\mathrm{dyn} \text {,disc }}$ its dynamical time. We calculate chemical enrichment using the instantaneous recycling approximation, so the rate of ejection of
Table 1. The parameters allowed to vary in our parameter space exploration. The first column provides an indication of the physical process associated with the parameter. The second column gives the parameter name; Column 3 gives the value of the parameter used in Bow06; and Columns 4 and 5 give the range of the parameter explored in this paper. Active variables in the model emulator (those that are important in capturing the behaviour of the $z=0$ luminosity function output of the model; see Section 3.5.2) are indicated in Column 6.

\begin{tabular}{lccccc}
\hline $\begin{array}{l}\text { Process } \\
\text { modelled }\end{array}$ & $\begin{array}{c}\text { Parameter } \\
\text { name }\end{array}$ & Bow06 & min & $\max$ & Active? \\
\hline Star formation & $\epsilon_{\star}^{-1}$ & 350 & 10 & 1000 & $\mathrm{~A}$ \\
& $\alpha_{\star}$ & -1.5 & -3.2 & -0.3 & $\mathrm{~A}$ \\
SNe feedback & $p_{\text {yield }}$ & 0.02 & 0.02 & 0.05 & $\mathrm{~A}$ \\
& $V_{\text {hot,disc }}$ & 485 & 100 & 550 & $\mathrm{~A}$ \\
& $V_{\text {hot,burst }}$ & 485 & 100 & 550 & $\mathrm{~A}$ \\
& $\alpha_{\text {hot }}$ & 3.2 & 2.0 & 3.7 & $\mathrm{~A}$ \\
AGN feedback & $\alpha_{\text {reheat }}$ & 0.92 & 0.2 & 1.2 & $\mathrm{~A}$ \\
& $\alpha_{\text {cool }}$ & 0.58 & 0.2 & 1.2 & $\mathrm{~A}$ \\
Galaxy mergers & $\epsilon_{\text {Edd }}$ & 0.04 & 0.004 & 0.05 & \\
& $f_{\text {df }}$ & 1.5 & 0.8 & 2.7 & $\mathrm{~A}$ \\
& $f_{\text {ellip }}$ & 0.3 & 0.1 & 0.35 & \\
& $f_{\text {burst }}$ & 0.1 & 0.01 & 0.15 & \\
Disc stability & $F_{\text {bh }}$ & 0.005 & 0.001 & 0.01 & \\
Reionization & $f_{\text {stab }}$ & 0.8 & 0.65 & 0.95 & $\mathrm{~A}$ \\
& $v_{\text {cut }}$ & 50 & 20 & 50 & \\
\hline & $z_{\text {cut }}$ & 6 & 6 & 9 & \\
\hline
\end{tabular}

newly synthesized metals into the ISM is given by

$\dot{M}_{\mathrm{Z}, \mathrm{ej}}=p_{\text {yield }} \mathrm{SFR}$

where $p_{\text {yield }}$ is the yield of metals, which depends on the IMF. For consistency with Bow06, we use a Kennicutt (1983) IMF throughout, but treat $p_{\text {yield }}$ as an adjustable parameter. In Font et al. (2008) and Bower et al. (2008), we showed that the match to the observed colours of galaxies was improved by adopting a higher yield (0.04) than the standard value (0.02).

The second group of parameters is associated with the supernovadriven feedback: $V_{\text {hot,disc }}$ and $V_{\text {hot,burst }}$ control the normalization of feedback in quiescent star formation and bursts, respectively; $\alpha_{\text {hot }}$ controls the dependence of the feedback on the circular velocity. For example, the rate at which mass is returned from the cold phase to the halo during quiescent star formation is given by

$\dot{M}_{\text {outflow }}=\operatorname{SFR}\left(\frac{V_{\text {c,disc }}}{V_{\text {hot,disc }}}\right)^{-\alpha_{\text {hot }}}$.

Cold gas that is ejected from the disc becomes available to cool and form further stars after a factor $\alpha_{\text {reheat }}{ }^{-1}$ times the halo dynamical time. In low-mass haloes cooling is very rapid, and this parameter plays a key role in setting the disc fuelling rate.

AGN feedback is controlled by the parameters $\alpha_{\text {cool }}$, which effectively determines the halo mass at which this form of feedback becomes effective, and $\epsilon_{\mathrm{Edd}},{ }^{1}$ which controls the maximum energy output possible for a central supermassive black hole of given Eddington luminosity $L_{\text {Edd }}$. Specifically, we only allow the AGN to regulate cooling if

$t_{\text {cool }}\left(r_{\text {cool }}\right)>\alpha_{\text {cool }}^{-1} t_{\text {ff }}\left(r_{\text {cool }}\right)$

\footnotetext{
${ }^{1}$ Note that due to an error in Bow06, cooling luminosities were overestimated by a factor $4 \pi$. Thus, while the paper quotes the efficiency parameter $\epsilon_{\mathrm{SMBH}}$ as 0.5 , this should have been $0.5 / 4 \pi=0.04$. With this correction the results of Bow06 are unchanged.
} 
and

$L_{\text {cool }}<\epsilon_{\text {Edd }} L_{\text {Edd }}$,

where $L_{\text {cool }}$ is the radiative cooling luminosity of the halo gas. Note that larger values of $\alpha_{\text {cool }}$ result in AGN feedback being effective in lower mass haloes.

Galaxy mergers are dependent on the rate of decay of satellite orbits due to dynamical friction and on the mass ratio of the merging objects. The normalization of the orbital decay rate is set by $f_{\mathrm{df}}$ (see Cole et al. 2000), while $f_{\text {ellip }}$ and $f_{\text {burst }}$ are, respectively, the mass ratios needed to transform the morphology of the main galaxy and to cause a burst of star formation (see Baugh et al. 2005; Malbon et al. 2007). ${ }^{2}$ The disc stability parameter, $f_{\text {stab }}$, sets the self-gravity threshold at which galaxy discs become unstable to bar modes (see Bow06). This instability causes the cold disc gas to be consumed in a burst of star formation. Smaller values of this parameter make discs more prone to bar instabilities.

Finally, the parameters $v_{\text {cut }}$ and $z_{\text {cut }}$ encapsulate the effect of reionization on cooling in small haloes. For further discussion of this approximation, see Benson et al. (2003b). We will show that these parameters have little impact on the galaxy properties we consider here.

We list in Table 1 the GALFORM parameters which we allow to vary in our parameter space exploration, together with their values in the Bow06 model and the ranges over which we allow them to vary. Ideally, we would know in advance what range for each parameter is physically meaningful or interesting, but this is only possible for a subset of the parameters. For example, the parameters $f_{\text {ellip }}$ and $f_{\text {burst }}$ are constrained to lie in the range $[0,1]$ by the way they are defined, and numerical simulations of merging galaxies constrain their values to an even narrower range. Similar arguments can be applied to restrict the range of $p_{\text {yield }}, \epsilon_{\mathrm{Edd}}, f_{\mathrm{df}}, f_{\mathrm{stab}}, v_{\text {cut }}$ and $z_{\text {cut }}$. On the other hand, theory does not currently provide any useful guide as to the value of $\epsilon_{\star}$, so the value of this parameter is set purely by comparison with observations and previous experience with GALFORM. In these cases, we selected the range by posing the question 'if an acceptable model was found outside this range, would it be interesting?' We answered no if the parameter value seemed inconsistent with the physical model that component of the code was intended to describe. The range selected is intended to be conservatively large, but is inevitably subjective. In some cases the parameter value adopted in Bow06 is uncomfortably high (e.g. the $V_{\text {hot,burst }}$ and $\alpha_{\text {hot }}$ parameters are in principle constrained by the amount of energy available from supernova explosions) and we deliberately extended the search range in order to bracket the value from Bow06.

In the following analysis, it is often helpful to use scaled variables so that each parameter covers the range \pm 1 . We denote scaled variables by $\tilde{\alpha}$ (etc.) where

$\tilde{\alpha}=\frac{\alpha-\frac{1}{2}\left(\alpha_{\max }+\alpha_{\min }\right)}{\frac{1}{2}\left(\alpha_{\max }-\alpha_{\min }\right)}$.

The end result is that the model spans a 16D parameter space. However, it is extremely important to stress that several of these parameters have little impact on the GALFORM output for the selected observables, and thus that it is initially possible for the emulator to capture the behaviour of the model using many fewer parameters.

\footnotetext{
${ }^{2}$ We note, however, that the parameter $f_{\text {gas, burst }}$ is set to 0.1 in this study, and in Bow06, so that almost all sufficiently high mass ratio mergers result in a burst of star formation. In Malbon et al. this parameter was set to 0.75 .
}

Our first step was to identify the most important parameters whose values were key to matching the selected galaxy properties. In terms of the match to the $b_{\mathrm{J}}$ and $K$ luminosity functions, there are 10 active parameters (at Wave 4, see Section 3.5.2) that drive the majority of the variation in model outputs. These are indicated by an A in Column 4 of Table 1 . As we will show, the parameter space of acceptable models is limited to a very small fraction of this volume. Even though adequate fits can be obtained for a wide range of parameter values, variations in parameters must be carefully traded off to keep the input parameter set on a narrow hypersurface.

Note, however, that a parameter that is inactive when the model is constrained using the luminosity function data may play an important role in fitting other data sets. For example, while the reionization parameters $v_{\text {cut }}$ and $z_{\text {cut }}$ have little effect on the global luminosity function (within the limits considered), these parameters play a key role in determining the satellite galaxy population of the Milky Way (Benson et al. 2003b).

\section{THE MODEL EMULATOR TECHNIQUE}

\subsection{Bayesian analysis of computer models}

There has been much interest in the statistics community in developing techniques to help understand and analyse complex computer simulations of real world processes, referred to generically as Computer Models (Currin et al. 1991; Craig et al. 1997; Santner, Williams \& Notz 2003; O'Hagan 2006). Such models, of which GALFORM is an example, generally take a significant time to run and require the specification of a large number of input parameters. They involve several distinct sources of uncertainty, all of which need to be assessed and combined in a unified analysis. These fall into five basic types.

(1) Parameter uncertainty: we do not know the appropriate values of the inputs to the simulator, and want to identify the class of inputs that give acceptable matches to the observed data.

(2) Simulator uncertainty: due to the significant run time we cannot hope to cover the input space with a suitably large number of model evaluations. Therefore, we will be uncertain as to the output of the model for regions of the input space where no evaluations have been performed. This uncertainty is handled through the use of an emulator as described in Section 3.2.

(3) Structural uncertainty: this aspect, which is less familiar to the astronomical community, refers to the fundamental problem that, however carefully the model has been constructed, there will always be a difference between the system (in this case the Universe) and the simulator. Simplifications in the physics, based on features that are too complicated for us to include, and simplifications and approximations in solving the equations determining the system, lead to a discrepancy between the model and the system. We represent this through use of the 'model discrepancy' term described in Section 3.6.

(4) Observational error: we do not know the properties of the real Universe exactly, but instead have observational measurements with corresponding errors.

(5) Initial condition and forcing function uncertainty: most Computer Models require the specification of initial conditions and/or forcing functions, the form of which is most likely uncertain.

In order to analyse the input space of the GALFORM model, and to determine which inputs are of interest, we need to address all of the above five sources of uncertainty in a unified manner. A Bayesian 
approach provides a natural framework for such an analysis. Powerful Bayesian techniques, centred around the idea of emulation, have been developed in the Statistics community for such problems, and have been successfully applied to models in several scientific disciplines (e.g. Kennedy \& O'Hagan 2001; Oakley \& O'Hagan 2002; Higdon et al. 2004; O'Hagan 2006; Schneider et al. 2008; Heitmann et al. 2009). However, employing a fully probabilistic Bayesian analysis (where every uncertain quantity is assigned a probability distribution) is often unnecessarily challenging and involves specifying prior distributions that are in some cases difficult to justify. Instead we employ the Bayes Linear approach (Goldstein \& Wooff 2007) which is a more tractable version of Bayesian analysis that requires fewer assumptions, and that deals with only expectations and variances of all uncertain quantities (see Section 3.5.4). The Bayes Linear methods presented here have been successfully applied to several complex models including Oil Reservoir Models and Climate Models (Craig et al. 1997, Goldstein \& Rougier 2009), and are well suited to the case of high-dimensional models.

\subsection{General emulation strategy}

An emulator is a stochastic function that represents our beliefs about the behaviour of a deterministic function at input settings that are yet to be evaluated. Representing a model such as GALFORM as a function that maps a vector of inputs $\boldsymbol{x}$ to a vector of outputs $\boldsymbol{f}(\boldsymbol{x})$, an emulator would give, for each input parameter setting $\boldsymbol{x}$, quantities such as an expectation and variance of the function: $\mathrm{E}(\boldsymbol{f}(\boldsymbol{x}))$ and $\operatorname{Var}(\boldsymbol{f}(\boldsymbol{x}))$. In this way it represents the expected value of the function at $x$ but also gives a measure of our uncertainty at this point through $\operatorname{Var}(f(\boldsymbol{x}))$. This uncertainty would be small at points close to known model runs, and large at points far from known runs. The expectation of the emulator will (in most cases, but not always) interpolate the outputs of evaluated runs.

Emulators have many advantages, the most important being their speed: in many cases an emulator will be many orders of magnitude faster than the model it represents. Also, emulators are designed to cope with high numbers of input dimensions, far more than can be handled by more traditional methods such as MCMCs (e.g. Heitmann et al. 2009).

Here, we use emulation techniques to identify the set of all inputs $\boldsymbol{x}$ that will give rise to an acceptable match (with respect to

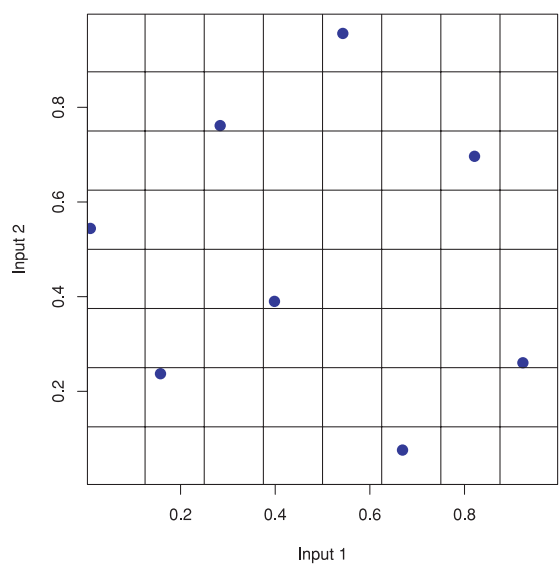

all relevant uncertainties) between the $b_{\mathrm{J}}$ and $K$ luminosity function outputs of GALFORM and the corresponding observational data [Norberg et al. (2002) for the $b_{\mathrm{J}}$ luminosity function and Cole et al. (2001) for the $K$ band].

The general strategy is as follows. Initially, we design a suitable set of 1000 runs of the GALFORM model chosen to be at parameter locations that will cover the input space efficiently, and help the construction of an acceptable emulator. Then, we identify a subset of 11 outputs (i.e. the predicted values of the luminosity function at selected magnitudes) which are representative of the $b_{\mathrm{J}}$ and $K$ luminosity functions, informative (about which regions of the input space are unacceptable) and that are also straightforward to emulate. We emulate each output by fitting a third-order polynomial (defined over the input space) to each of the 11 outputs, and then modelling the residuals of this fit as a Gaussian process. Using the emulators and assessments of all other relevant uncertainties (as described in Section 3.1) we then construct an Implausibility Measure defined over the input space (see Section 3.7). Regions of the input space that have a high Implausibility Measure are deemed highly unlikely to give an acceptable match between the luminosity function output of GALFORM and the observed data and are hence discarded from further analysis. This defines a reduced region of input space that we can explore further. We employ an iterative approach, and have reduced the input space in four stages as is described below.

\subsection{Designing the first set of runs}

Determining a highly informative collection of points in input space to perform evaluations of a Computer Model such as GALFORM is an important task. The points must be space filling in addition to avoiding repeated runs at similar values of one or more of the inputs (as occurs regularly in a standard grid design). Maximin Latin Hypercube (Stein 1987) designs fulfil both these properties and were used to generate the initial set of runs. These designs are also approximately orthogonal: a desirable property when trying to fit polynomials to a function, as is the case when building an emulator. To construct a Latin Hypercube of $n$ points, the range of each of the inputs must be divided into $n$ equal intervals; the points are then chosen randomly so that no two points occupy the same interval for any of the inputs. Examples of two-dimensional (2D) eight- and 20-point Latin Hypercube designs are shown in Fig. 1. A

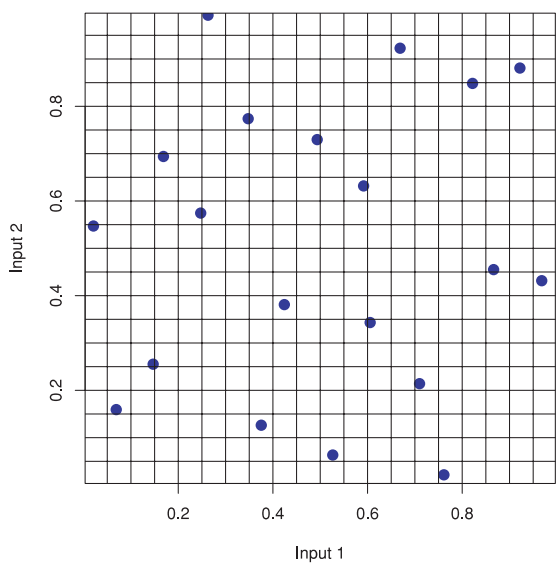

Figure 1. Two examples of Latin Hypercube designs for a 2D parameter space. The range of each input is divided into $n$ intervals where $n$ is the number of points. Note that only one point is placed in each of the $n$ intervals over Input 1 and Input 2, and that these points have been placed at random within each interval. The two panels show samplings with eight and 20 points. The Maximin strategy adopted in this paper ensures that the points are evenly spread throughout the region that we wish to sample. 
Maximin Latin Hypercube is constructed by creating a large number of Latin Hypercube designs, and then choosing the one that has the largest minimum distance between any pair of points within that design. For each design, we generated 2000 hypercubes and then selected the best one using the maximin criterion. 1000 such runs of the GALFORM model were performed based on such a Maximin Latin Hypercube design, and these runs form the basis of Wave 1 of our analysis.

\subsection{Choosing outputs}

Once the 1000 runs were completed, 11 outputs (i.e. the values of the luminosity function at selected magnitude points) were chosen for emulation: six from the $b_{\mathrm{J}}$ luminosity and five from the $K \mathrm{lu}$ minosity functions. These are shown as the vertical black dashed lines in Fig. 2, along with the full outputs from the 1000 runs and the observed data (the error bars contain all relevant uncertainties as discussed below). These particular 11 magnitude outputs were chosen as they represented the form of the luminosity functions well (and hence can be used to reconstruct the luminosity function), were easy to emulate and, most importantly, were also sensitive to changes in the input parameters implying that they are very informative with regard to the input space. This last point implies
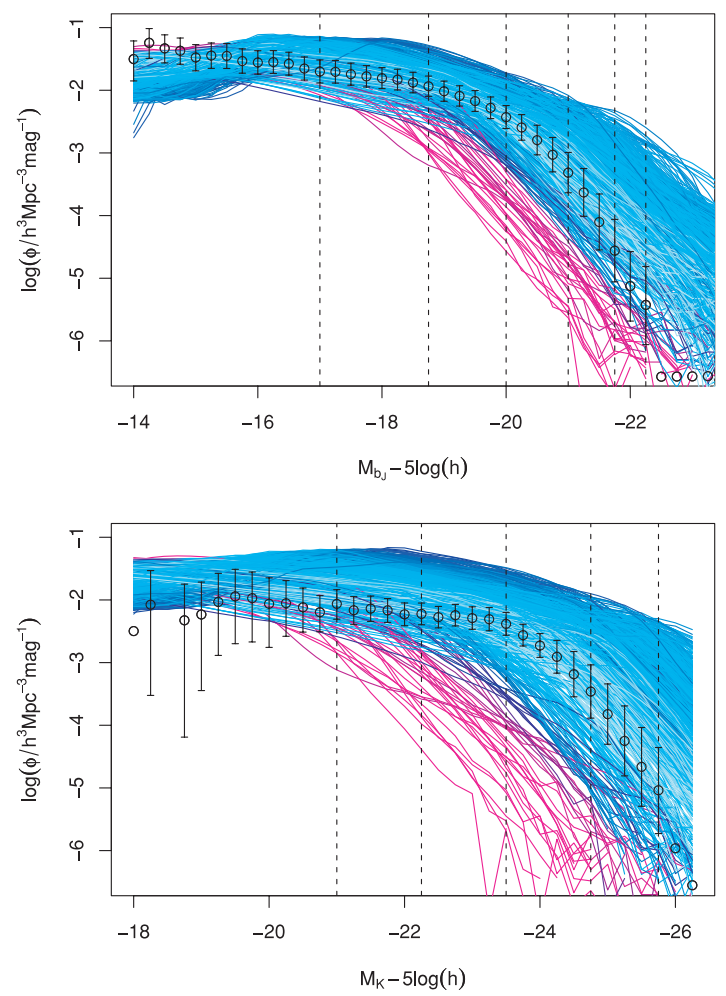

Figure 2. The $b_{\mathrm{J}}$ and $K$ luminosity functions from the first 1000 runs of the model (Wave 1) compared to observational data. The data, from Norberg et al. (2002) and Cole et al. (2001), respectively, are shown as black points with $2 \sigma$ error bars which include all observational and model discrepancy uncertainties as described in Section 3.6. Parameter values were chosen using a Maximin Latin Hypercube design spanning the eight most important parameters (see Table 2). The vertical dashed black lines show the 11 outputs chosen for emulation. These provide a good characterization of the luminosity function. Note that below $b_{\mathrm{J}}=16$, some luminosity function calculations are affected by numerical resolution. The colouring of lines indicates the quality of the match to the observed data, with blue colours indicating $I_{M}<16$. that we can reliably cut out regions of the input space using only these 11 outputs, and without being forced into emulating the luminosity function in every luminosity bin. The analysis of the initial runs showed that adding additional outputs did not significantly improve the characterization of the luminosity function, but did risk weighting Implausibility Measures too much towards the faint-end performance of a model. We also note that we did not attempt to emulate the luminosity function for $b_{\mathrm{J}}<16$ mag or $K<20$ mag because the limited resolution of the Millennium simulation becomes important for some parameter values in this region.

\subsection{Constructing the emulator}

\subsubsection{A simple example}

Before we describe the construction of the emulator for the GALFORM model, it is useful to briefly outline how the method might be applied to a simple one-dimensional (1D) problem.

The first step is to construct an emulator of the simple 1D function shown in Fig. 3. Imagine that the function, $f(x)$, is a one parameter model for some measurable quantity. In the left-hand panels, the function (which is in fact a simple sine wave) has been evaluated at $n=6$ input points denoted as $x_{i}$. We use the function output at these points, $k_{i}=f\left(x_{i}\right)$, to construct an emulator based on a random Gaussian process, $u(x)$, that is we say

$f(x)=u(x)$

where we assume the prior expectation and variance of the process $u(x)$ to be $\mathrm{E}(u(x))=0$ and $\operatorname{Var}(u(x))=\sigma^{2}$, and that the prior covariance structure is defined to be of Gaussian form with correlation length $\theta$ :

$c\left(x, x^{\prime}\right)=\operatorname{Cov}\left(u(x) u\left(x^{\prime}\right)\right)=\sigma^{2} \exp \left(-\left(x-x^{\prime}\right)^{2} / \theta^{2}\right)$.

We can now update the emulator $u(x)$ using the knowledge of the six evaluations of $f(x)$. The updated emulator at a new point $x^{\prime}$ now has expectation and variance given by

$\mathrm{E}\left[u\left(x^{\prime}\right)\right]=\boldsymbol{t}\left(x^{\prime}\right)^{T} \mathbf{A}^{-1} \boldsymbol{k}$

$\operatorname{Var}\left[u\left(x^{\prime}\right)\right]=\sigma^{2}-\boldsymbol{t}\left(x^{\prime}\right)^{T} \mathbf{A}^{-1} \boldsymbol{t}\left(x^{\prime}\right)$

where $\boldsymbol{k}=\left(f\left(x_{1}\right), f\left(x_{2}\right), \ldots, f\left(x_{n}\right)\right)^{T}$ (the vector of known function values), $t\left(x^{\prime}\right)=\left(c\left(x^{\prime}, x_{1}\right), c\left(x^{\prime}, x_{2}\right), \ldots, c\left(x^{\prime}, x_{n}\right)\right)^{T}$ (the column vector of covariances between the new and known points) and $\mathbf{A}$ is an $n \times n$ matrix with elements $A_{i j}=c\left(x_{i}, x_{j}\right)$ (the matrix of covariances between known points; e.g. Williams 2002). In a fully probabilistic analysis, equations (9) and (10) would be derived from conditioning a Gaussian Process on the six known evaluations, and would give the mean and variance of the corresponding Normal distribution of $u\left(x^{\prime}\right)$ at the input point $x^{\prime}$. However, here we use a Bayes Linear analysis where equations (9) and (10) are derived directly from the Bayes Linear update (described in Section 3.5.4 and given by equations 15 and 17), and are considered as primitive quantities which are used directly to assess whether parts of the input space are acceptable.

The random process $u\left(x^{\prime}\right)$ quantifies the uncertainty in this fit: close to points at which the function has been evaluated the uncertainty is small, while between points it is larger. Making a suitable choice for $\sigma$ and $\theta$ is problem specific. In this example, we set $\sigma$ to be 0.3 and chose $\theta$ to be $1 / 5$ of the range of the input variable $x$.

Now we suppose that we have some measurement for the quantity being modelled. This is shown by the horizontal black lines (thin lines indicating the measurement uncertainty) in the figure. Using 

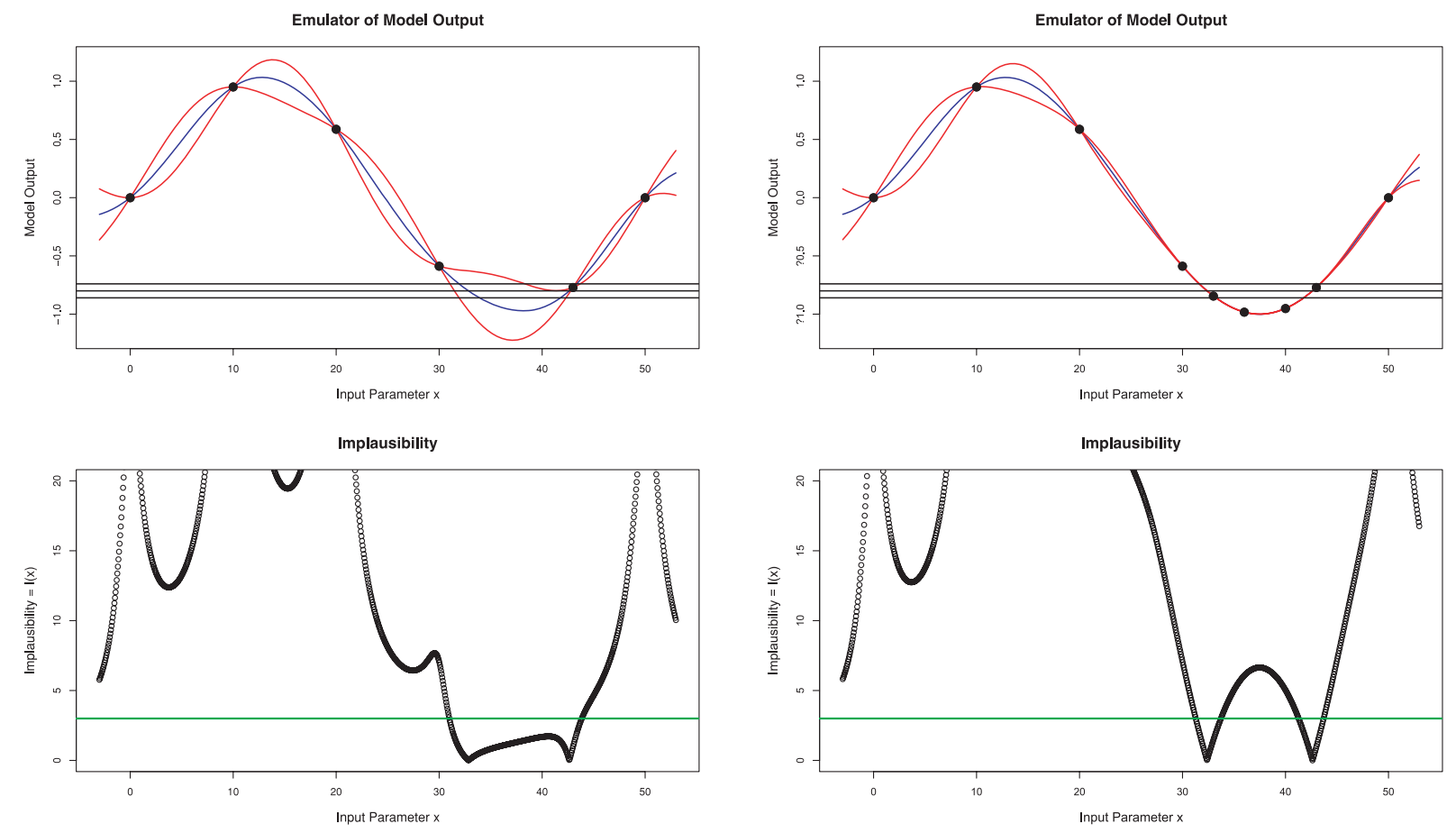

Figure 3. An example of the emulation of a 1D function (a sine wave). The top-left panel shows the emulator $f(x)=u(x)$ after six evaluations of the function: the blue line is the $\mathrm{E}(f(x))$, the two red lines define a credible interval of $\mathrm{E}(f(x)) \pm 2 \sqrt{\operatorname{Var}(f(x))}$ and the black dots are the six model outputs. Observational data $z$ is represented as the middle black line, with $2 \sigma$ errors given by the top and bottom black line. The bottom-left panel shows the implausibility function $I(x)$ in black, with the cut-off of three in green. Inputs along the $x$-axis are deemed implausible if $I(x)>3$. The top right and bottom right panels show the situation after three more runs have been performed in the non-implausible region. We re-emulate, and now, as the emulator is far more accurate, the implausibility naturally gives the two regions of the $x$-axis where the function matches the observed data (approximately around $x=33$ and $x=43$ ).

our emulator, we now try to identify the parameter values at which an evaluation of the model might be compatible with measured data. While our emulator cannot guarantee that an evaluation will successfully match the data, it identifies the regions at which a match is implausible. This is quantified through the use of an implausibility function, $I(x)$, which is discussed in more detail in Section 3.7. In this simple example $I(x)$ is defined as

$I^{2}(x)=|\mathrm{E}(f(x))-z|^{2} /\left(\operatorname{Var}(f(x))+\operatorname{Var}\left(\epsilon_{\mathrm{obs}}\right)\right)$,

where $z=-0.8$ is the observation (the middle horizontal black line), $\operatorname{Var}\left(\epsilon_{\mathrm{obs}}\right)$ is the variance of the observational errors which in this case were taken to be $0.03^{2}$, and $\mathrm{E}(f(x))$ and $\operatorname{Var}(f(x))$ are given by equations (9), (10) and (7).

The value of $I(x)$ is shown in the lower left-hand panel of Fig. 3: where $I(x)$ is large we reject the parameter values from further investigation. However, where $I(x)$ is below our cut-off value (in this case 3 ), we perform a small number of additional evaluations of $f$. We then re-emulate using these additional evaluations, and this is shown in the right-hand panels. Now the uncertainty in the critical region is much reduced and the only 'non-implausible' regions are closely centred on the regions where $f$ truly matches the measurement. This iterative approach is known as 'history matching' and is explained in more detail below, but it can be see that the technique allows us to focus our evaluations of $f$ on the regions where additional knowledge is critical.

Obviously, this 1D example is highly simplified, and an emulator is not required to solve this problem. In our GALFORM application we are aiming to search a much higher dimensional parameter space and the ability to quantify our knowledge of the model is critical. Further, evaluation of the emulator is almost $10^{7}$ times faster than running an individual model, and thus this technique can be combined with the Latin Hypercube scheme to explore a seemingly vast parameter space. Note that for the simple example we have used an emulator consisting of purely a Gaussian Process, given by equation (7). When we come to emulate the GALFORM model we will use a more advanced emulator that contains polynomial regression terms, a Gaussian Process to model the residuals of the regression and a white noise term to model ineffective variables. In the sections below, we describe the construction of the GALFORM emulator in more detail.

\subsubsection{The GALFORM emulator}

Here, we describe the construction of the emulators for each of the 11 outputs identified in Section 3.3. It must be stressed that although we provide some detail, the construction of the emulators and the subsequent analysis involve an extensive collection of statistical techniques, and we cannot hope to give a comprehensive treatment here. A much more extensive description is given in Vernon et al. (2010). For examples of such techniques used in other applications see Craig et al. (1996) and Goldstein \& Rougier (2009).

We view the GALFORM model as a function that maps the 16 inputs in Table 1 to the 11 identified outputs, and denote it by $\boldsymbol{f}(\boldsymbol{x})$ where $\boldsymbol{f}$ is an 11 component vector of outputs and $\boldsymbol{x}$ a 16 component vector of inputs with $\boldsymbol{x}=\left(x_{1}, x_{2}, x_{3}, \ldots\right)=\left(V_{\text {hot,disc }}, \alpha_{\text {reheat }}, \alpha_{\text {cool }}, \ldots\right)$. (Note that we use the scaled variables directly, without taking logs even when the variables cover a large range.) Great simplifications can be made in the construction of emulators through the use of active variables. Often, a subset of the inputs has strong effects on the outputs that are to be emulated and we call these the Active Variables (see Table 1). We define $\boldsymbol{x}_{A}$ to be a vector composed of 
active variables only and model their effects on $\boldsymbol{f}(\boldsymbol{x})$ in detail (Craig 1996). The remaining inputs (the inactive variables) have only minor effects on the outputs so are treated as contributing a noise process to the emulator. The form of the emulator for component $i$ of $\boldsymbol{f}(\boldsymbol{x})$ would then be

$f_{i}(\boldsymbol{x})=\sum_{j} \beta_{i j} g_{i j}\left(\boldsymbol{x}_{A}\right)+u_{i}\left(\boldsymbol{x}_{A}\right)+w_{i}(\boldsymbol{x})$.

Here the $g_{i j}\left(\boldsymbol{x}_{A}\right)$ are known functions chosen to be first-, secondor third-order polynomial terms in the active variables [for example, for output $i=1$ we might have terms of the form $g_{1 j}\left(\boldsymbol{x}_{A}\right)=$ $x_{1} x_{2}^{2}, x_{3}^{3}$ or $x_{1} x_{2} x_{3}$, with different terms corresponding to different values of $j$ ]; the $\beta_{i j}$ are coefficients of the polynomial which will be fitted using regression methods. $u_{i}\left(\boldsymbol{x}_{A}\right)$ is a Gaussian Process ${ }^{3}$ which also depends only on the active variables. The effects of the inactive parameters are described by the $w_{i}(\boldsymbol{x})$ term, referred to as a nugget, which is modelled as a random white noise process. The regression term $\sum_{j} \beta_{i j} g_{i j}\left(\boldsymbol{x}_{A}\right)$ on the right-hand side of equation (12) is included to capture the global behaviour of the GALFORM function. The Gaussian process $u\left(\boldsymbol{x}_{A}\right)$ represents localized deviations from this global behaviour, and a simple specification is to suppose, for each $x$, that $u_{i}(x)$ has zero mean, constant variance and $\operatorname{Cov}\left(u_{i}(x) u_{i}\left(x^{\prime}\right)\right)$ which is a function of $\left\|x-x^{\prime}\right\|$, here chosen to be of Gaussian form (see equation 8). As we perform evaluations of the model, the expectation and variance of $f_{i}(\boldsymbol{x})$ at a given point is then updated using the Bayes Linear analysis as described in Section 3.5.4.

The above describes the general structure for all the emulators used in this analysis. As we perform the reduction of input space iteratively, and at each iteration (or 'wave') we re-emulate changing the specific form for the emulators (see the section on History Matching below; Section 3.8). At each wave the number of active variables increases as the emulator becomes more accurate, and the random processes $u_{i}\left(\boldsymbol{x}_{A}\right)$ and $w_{i}(\boldsymbol{x})$ become less significant.

\subsubsection{The Wave 1 emulator}

Here we outline the construction of the Wave 1 emulators (full details, and extensive discussion, are given in Vernon et al. 2010). First, eight of the 16 inputs were chosen as candidate active variables due to their clear effect on the luminosity output in a set of initial test runs. In choosing the active variables, the aim is to explain a large amount of the variance of $f_{i}(\boldsymbol{x})$ using as few variables as possible. Initially, we ran GALFORM varying the primary parameters and holding the others fixed at their central values. The effect of the fixed variables is accounted for through a contribution to the model discrepancy term (see Section 3.6). (Note that in Wave 4, as the region of parameter space becomes more restricted, we will allow the full set of parameters to vary.) For each of the 11 outputs, the set of eight parameters was initially reduced by backwards stepwise elimination, starting with a model containing the eight linear terms in $\boldsymbol{x}$. Then, individual inputs were discarded in turn based upon the significance of their main (i.e. linear) effect. Before an input would be discarded, a full third-order polynomial was fitted to see the extent of variance explained with the current set of active variables. It was found that five active variables could explain satisfactory amounts of the variance of $f_{i}(\boldsymbol{x})$ for each output $i$, based on the adjusted $R^{2}$ of the polynomial fits $\left(R^{2}\right.$ is the Coefficient of

\footnotetext{
${ }^{3}$ Technically, we should refer to $u_{i}\left(\boldsymbol{x}_{A}\right)$ as a weakly stationary random process in the Bayes Linear context, as we are making no assertions regarding the behaviour of higher order moments of $u_{i}\left(\boldsymbol{x}_{A}\right)$.
}

Table 2. The eight candidate active variables for Wave $1 b_{\mathrm{J}}$ luminosity function output (see Fig. 2), with the final five active variables for each $b_{\mathrm{J}}$ output marked by an $\mathrm{x}$. The adjusted $R^{2}$ value gives a measure of how effective the third-order polynomial is in capturing the global behaviour of the particular $b_{\mathrm{J}}$ luminosity GALFORM output. Note the low adjusted $R^{2}$ of outputs 3 and 4 : these were not used in the first wave analysis, but did feature in later waves.

\begin{tabular}{lcccccc}
\hline Output & $b_{\mathrm{J}}^{1}$ & $b_{\mathrm{J}}^{2}$ & $b_{\mathrm{J}}^{3}$ & $b_{\mathrm{J}}^{4}$ & $b_{\mathrm{J}}^{5}$ & $b_{\mathrm{J}}^{6}$ \\
\hline$V_{\text {hot,disc }}$ & $\mathrm{x}$ & $\mathrm{x}$ & $\mathrm{x}$ & $\mathrm{x}$ & $\mathrm{x}$ & $\mathrm{x}$ \\
$\alpha_{\text {reheat }}$ & $\mathrm{x}$ & $\mathrm{x}$ & $\mathrm{x}$ & $\mathrm{x}$ & $\mathrm{x}$ & $\mathrm{x}$ \\
$\alpha_{\text {cool }}$ & & & $\mathrm{x}$ & $\mathrm{x}$ & $\mathrm{x}$ & $\mathrm{x}$ \\
$V_{\text {hot,burst }}$ & $\mathrm{x}$ & & $\mathrm{x}$ & $\mathrm{x}$ & $\mathrm{x}$ & $\mathrm{x}$ \\
$\epsilon_{\star}$ & $\mathrm{x}$ & $\mathrm{x}$ & $\mathrm{x}$ & $\mathrm{x}$ & & \\
$f_{\text {stab }}$ & & & & & $\mathrm{x}$ & $\mathrm{x}$ \\
$\alpha_{\text {hot }}$ & $\mathrm{x}$ & $\mathrm{x}$ & & & & \\
$p_{\text {yield }}$ & & $\mathrm{x}$ & & & & \\
Adj. $R^{2}$ & 0.92 & 0.71 & 0.55 & 0.59 & 0.71 & 0.70 \\
\hline
\end{tabular}

Table 3. The eight candidate active variables for the Wave $1 K$-band luminosity function output (see Fig. 2), with the final five active variables for each $K$-band output marked by an x. Note the low adjusted $R^{2}$ of output 3 : this was not used in the first wave analysis, but did feature in later waves.

\begin{tabular}{lccccc}
\hline Output & $K^{1}$ & $K^{2}$ & $K^{3}$ & $K^{4}$ & $K^{5}$ \\
\hline$V_{\text {hot,disc }}$ & $\mathrm{x}$ & $\mathrm{x}$ & $\mathrm{x}$ & $\mathrm{x}$ & $\mathrm{x}$ \\
$\alpha_{\text {reheat }}$ & $\mathrm{x}$ & $\mathrm{x}$ & $\mathrm{x}$ & $\mathrm{x}$ & $\mathrm{x}$ \\
$\alpha_{\text {cool }}$ & & & & $\mathrm{x}$ & $\mathrm{x}$ \\
$V_{\text {hot, burst }}$ & $\mathrm{x}$ & $\mathrm{x}$ & $\mathrm{x}$ & $\mathrm{x}$ & $\mathrm{x}$ \\
$\epsilon_{\star}$ & $\mathrm{x}$ & $\mathrm{x}$ & $\mathrm{x}$ & $\mathrm{x}$ & $\mathrm{x}$ \\
$f_{\text {stab }}$ & $\mathrm{x}$ & $\mathrm{x}$ & $\mathrm{x}$ & & \\
$\alpha_{\text {hot }}$ & & & & & \\
$p_{\text {yield }}$ & & 0.75 & 0.61 & 0.72 & 0.80 \\
Adj. $R^{2}$ & 0.87 & & & & \\
\hline
\end{tabular}

Determination of the fit and takes values between 0 and 1 , with higher values implying that the fit explains more of the model output's behaviour). Note that the subset of five active variables is in general different for each output variable, as shown in Tables 2 and 3. Including more than five variables would yield little extra benefit at this stage, while using fewer than five leads to a significantly worse description of the main trends.

Once the set of active variables has been determined, the full set of regression terms [the $\beta_{i j} g_{i j}\left(\boldsymbol{x}_{A}\right)$ ] can be chosen. This was done by starting with the full third-order polynomial in the five active variables and using backwards stepwise elimination to remove less significant terms from the model. Note that a large number of model evaluations are required to enable the fitting of a third-order polynomial, although this greatly depends on the number of active variables. Now that the final regression terms have been chosen for each output $f_{i}(\boldsymbol{x})$, estimates for the set of $\left\{\beta_{i j}\right\}$ coefficients can be obtained using Ordinary Least Squares, assuming uncorrelated errors (a reasonable assumption as we have a large number of runs sufficiently far apart in the input space that the residuals should not be strongly correlated). Note that it is important to check that the structure of the polynomials obtained from this process agree with physical intuition.

For the two contributions to the residual process $u_{i}\left(\boldsymbol{x}_{A}\right)$ and $w_{i}(\boldsymbol{x})$ we specify a correlation structure as follows. As the $u_{i}\left(\boldsymbol{x}_{A}\right)$ represent local deviations from the regression surface we assume that there will be a large correlation between $u_{i}$ at neighbouring values of 
the active inputs $\boldsymbol{x}_{A}$, and specify the following Gaussian covariance structure:

$\operatorname{Cov}\left(u_{i}\left(\boldsymbol{x}_{A}\right) u_{i}\left(\boldsymbol{x}_{A}^{\prime}\right)\right)=\sigma_{u_{i}}^{2} \exp \left(-\left\|\boldsymbol{x}_{A}-\boldsymbol{x}_{A}^{\prime}\right\|^{2} / \theta_{i}^{2}\right)$,

where $\sigma_{u_{i}}^{2}$ is the point variance at any given $\boldsymbol{x}_{A}, \theta_{i}$ is the correlation length parameter that controls the strength of correlation between two separated points in the input space [for points a distance $\theta$ apart, the correlation will be exactly $\exp (-1)]$, and $\|\cdot\|$ is the Euclidean norm. As the nugget process $w_{i}(\boldsymbol{x})$ represents all the remaining variation in the inactive variables, it is often small and we treat it as uncorrelated random noise with $\operatorname{Var}\left(w_{i}(\boldsymbol{x})\right)=\sigma_{w_{i}}^{2}$. We consider the point variances of these two processes to be proportions of the overall residual variance: $\sigma_{i}^{2}$ (which is obtained from the OLS regression fit), and write that $\sigma_{u_{i}}^{2}=\left(1-\delta_{i}\right) \sigma_{i}^{2}$ and $\sigma_{w_{i}}^{2}=\delta_{i} \sigma_{i}^{2}$ for some usually small $\delta_{i}$.

Various techniques for estimating the correlation length and nugget parameters $\theta_{i}$ and $\delta_{i}$ from the data are available (e.g. variograms, REML, maximum likelihood; Cressie 1991), however an alternative is to specify them from previous experience of computer models (Craig et al. 2001, Kennedy01) which is the approach we adopt here. Specifically, in Wave 1 we choose $\theta_{i}=0.35$ and $\delta=0.2$, remembering that the inputs have all been scaled so that their range is $[-1,1]$. The choice for theta is motivated by the fact that we are fitting third-order polynomials to the model output, and therefore the residuals from this fit, which are modelled by the stationary process, will behave like a fourth (or higher) order polynomial. This suggests a correlation length of 0.35 would be reasonable. The value for $\delta$ is assessed by examining the variance of the model output explained by the inactive variables by fitting various polynomials using only the inactive variables. It should be noted that the choices for $\theta$ and $\delta$ are more conservative than values obtained using alternative estimation techniques, and that this was a deliberately cautious choice. More details of the motivation of these parameter choices, and description of the diagnostic used to confirm the accuracy of this approach, are given in Vernon et al. (2010).

The next step is to update the process $f_{i}(\boldsymbol{x})$ at a new point $\boldsymbol{x}$, with the information contained in the 1000 runs of the model. We do this using the Bayes Linear update formula discussed in the next section.

\subsubsection{Bayes linear approach}

For large-scale problems involving computer models such as GALFORM, a full Bayes analysis (involving probability distributions for all random quantities) is difficult for the following reasons. First, it is very difficult to give a meaningful full prior probability specification over high-dimensional input spaces. Secondly, the computations for learning from both observed data and runs of the model, and choosing informative runs, may be technically very challenging. Thirdly, in such computer model problems, often the likelihood surface is extremely complicated, and therefore any full Bayes calculation may be extremely non-robust. However, the basic idea of capturing our expert prior judgments in stochastic form and modifying them by appropriate rules given observations, is conceptually appropriate.

The Bayes Linear approach is (relatively) simple in terms of belief specification and analysis, as it is based only on the mean, variance and covariance specification which, following De Finetti (1974), we take as primitive. Therefore, a Bayes Linear approach proceeds by the specification and modification of mean and variance structures only.
We replace Bayes Theorem (which deals with full probability distributions) by the Bayes Linear adjustment which is the appropriate updating rule for expectations and variances. The Bayes Linear adjustment of the mean and the variance of a random quantity $B$ given data $D$ is

$\mathrm{E}_{D}[B]=\mathrm{E}(B)+\operatorname{Cov}(B D) \operatorname{Var}(D)^{-1}(D-\mathrm{E}(D))$,

$\operatorname{Var}_{D}[B]=\operatorname{Var}(B)-\operatorname{Cov}(B, D) \operatorname{Var}(D)^{-1} \operatorname{Cov}(D, B)$.

$\mathrm{E}_{D}[B], \operatorname{Var}_{D}[B]$ are the expectation and variance for $B$ adjusted by knowledge of $D .{ }^{4}$ In equations (14) and (15), $B$ and $D$ can represent scalars or vectors of uncertain quantities. In the latter case (15) and (17) become matrix equations where if $B$ is a vector of length $n_{B}$ and $D$ is a vector of length $n_{D}, \operatorname{Cov}(B, D)$ is a matrix of dimension $n_{B} \times n_{D}$ and $\operatorname{Var}(D)$ a matrix of dimension $n_{D} \times n_{D}$.

The Bayes linear adjustment may be viewed as an approximation to a full Bayes analysis, or more fundamentally as the 'appropriate' analysis given a partial prior specification based on expectation. For more details see Goldstein \& Wooff (2007).

\subsubsection{Updating the emulator}

Equations (14) and (15) give the rule for updating the emulator with the knowledge of the 1000 model evaluations, where the random quantities $B$ and $D$ will represent an unknown output and the collection of 1000 known outputs, respectively. We proceed with the update as follows. As we have a relatively large number of runs, we first assume that the regression coefficients $\beta_{i j}$ in the emulator equation (12) are known and hence have zero variance. As $u_{i}\left(\boldsymbol{x}_{A}\right)$ and $w_{i}(\boldsymbol{x})$ both have zero expectation, equation (12) gives the expectation of model output $i$ at input $\boldsymbol{x}$ to be

$\mathrm{E}\left(f_{i}(\boldsymbol{x})\right)=\sum_{j} \beta_{i j} g_{i j}\left(\boldsymbol{x}_{A}\right)$

and the variance of $f_{i}(\boldsymbol{x})$ to be

$\operatorname{Var}\left(f_{i}(\boldsymbol{x})\right)=\operatorname{Var}\left(u_{i}\left(\boldsymbol{x}_{A}\right)\right)+\operatorname{Var}\left(w_{i}(\boldsymbol{x})\right)=\sigma_{u_{i}}^{2}+\sigma_{w_{i}}^{2}=\sigma_{i}^{2}$.

As the $u_{i}\left(\boldsymbol{x}_{A}\right)$ and the $w_{i}(\boldsymbol{x})$ terms are uncorrelated, the covariance between output $i$ at two different inputs $\boldsymbol{x}^{\prime}$ and $\boldsymbol{x}$ can now be written (using equations 12 and 13) as

$$
\begin{aligned}
c\left(\boldsymbol{x}^{\prime}, \boldsymbol{x}\right) & =\operatorname{Cov}\left(f_{i}\left(\boldsymbol{x}^{\prime}\right) f_{i}(\boldsymbol{x})\right) \\
& =\operatorname{Cov}\left(u_{i}\left(\boldsymbol{x}_{A}^{\prime}\right) u_{i}\left(\boldsymbol{x}_{A}\right)\right)+\operatorname{Cov}\left(w_{i}\left(\boldsymbol{x}^{\prime}\right) w_{i}(\boldsymbol{x})\right) \\
& =\sigma_{u_{i}}^{2} \exp \left(-\left\|\boldsymbol{x}_{A}^{\prime}-\boldsymbol{x}_{A}\right\|^{2} / \theta_{i}^{2}\right)+\sigma_{w_{i}}^{2} \delta_{x^{\prime} \boldsymbol{x}}
\end{aligned}
$$

where $\delta_{x^{\prime} \boldsymbol{x}}$ is a Kronecker delta, equal to 1 when $\boldsymbol{x}^{\prime}=\boldsymbol{x}$ and zero otherwise. The second term in equation (18) comes from the nugget $w_{i}(\boldsymbol{x})$ which gives a zero contribution except when $\boldsymbol{x}^{\prime}=\boldsymbol{x}$.

We can now define the following quantities corresponding to the $n=1000$ model evaluations. We write the locations of the $n$ runs in input space as $\boldsymbol{x}_{j}$ with $j=1, \ldots, n$ where each $\boldsymbol{x}_{j}$ represents the vector of inputs for the $j$ th run. Similarly $\boldsymbol{x}_{A, j}$ is defined to be the vector of Active Variable inputs for the $j$ th run. We define $D_{i}=\left(f_{i}\left(\boldsymbol{x}_{1}\right), f_{i}\left(\boldsymbol{x}_{2}\right), \ldots, f_{i}\left(\boldsymbol{x}_{n}\right)\right)^{T}$, that is the column vector of the $n$ evaluation outputs for output $i$, the prior expectation of which $\left(\mathrm{E}\left(D_{i}\right)\right)$ can be found using equation (16).

\footnotetext{
${ }^{4} \mathrm{E}_{D}[B]$ and $\operatorname{Var}_{D}[B]$ are the corresponding Bayes Linear quantities to $\mathrm{E}(B \mid D)$ and $\operatorname{Var}(B \mid D)$, the conditional expectation and variance of $B$ given data $D$ that would be extracted from a fully Bayesian analysis.
} 
Replacing the random quantity $B$ in the Bayes Linear update equation (15) with the unknown output $f_{i}(\boldsymbol{x})$ at input $\boldsymbol{x}$ gives the adjusted expectation $\mathrm{E}_{D_{i}}\left[f_{i}(\boldsymbol{x})\right]$ to be

$\mathrm{E}_{D_{i}}\left[f_{i}(\boldsymbol{x})\right]=\mathrm{E}\left(f_{i}(\boldsymbol{x})\right)+\operatorname{Cov}\left(f_{i}(\boldsymbol{x}) D_{i}\right) \operatorname{Var}\left(D_{i}\right)^{-1}\left(D_{i}-\mathrm{E}\left(D_{i}\right)\right)$,

which becomes (using equation 19)

$\mathrm{E}_{D_{i}}\left[f_{i}(\boldsymbol{x})\right]=\sum_{j} \beta_{i j} g_{i j}\left(\boldsymbol{x}_{A}\right)+\boldsymbol{t}(\boldsymbol{x})^{T} A^{-1}\left(D_{i}-\mathrm{E}\left(D_{i}\right)\right)$,

where now $\boldsymbol{t}(\boldsymbol{x})=\left(c\left(\boldsymbol{x}, \boldsymbol{x}_{1}\right), c\left(\boldsymbol{x}, \boldsymbol{x}_{2}\right), \ldots, c\left(\boldsymbol{x}, \boldsymbol{x}_{n}\right)\right)^{T}=\operatorname{Cov}\left(f_{i}(\boldsymbol{x}) D_{i}\right)$ is the column vector of covariances between the new and known points, and $A$ is the matrix of covariances between known points: an $n \times n$ matrix with elements $A_{j k}=c\left(\boldsymbol{x}_{j}, \boldsymbol{x}_{k}\right)$. The Adjusted Variance $\operatorname{Var}_{D_{i}}[B]$ can similarly be found from equation (15) and (17) giving

$$
\begin{aligned}
& \operatorname{Var}_{D_{i}}\left[f_{i}(\boldsymbol{x})\right] \\
& \quad=\operatorname{Var}\left(f_{i}(\boldsymbol{x})\right)-\operatorname{Cov}\left(f_{i}(\boldsymbol{x}) D_{i}\right) \operatorname{Var}\left(D_{i}\right)^{-1} \operatorname{Cov}\left(D_{i} f_{i}(\boldsymbol{x})\right), \\
& \quad=\sigma_{i}^{2}-\boldsymbol{t}(\boldsymbol{x})^{T} A^{-1} \boldsymbol{t}(\boldsymbol{x}) .
\end{aligned}
$$

The Adjusted Expectations and Variances, $\mathrm{E}_{D_{i}}\left[f_{i}(x)\right]$ and $\operatorname{Var}_{D_{i}}[B]$, given by equations (19) and (20) form the basic ingredients in the construction of the Implausibility Measure used to reduce the input space to a much smaller 'non-implausible' volume. Note that if we had chosen a simple emulator of the form $f_{i}(\boldsymbol{x})=u_{i}(\boldsymbol{x})$, such as was used in the 1D example in Section 3.5.1, then equations (19) and (20) would reproduce exactly equations (9) and (10).

\subsection{Linking the model to the system: structural uncertainty and model discrepancy}

In order to declare regions of the input space as 'implausible', and to then exclude them from the analysis, we need to formally link the GALFORM model $\boldsymbol{f}(\boldsymbol{x})$ to the observed luminosity function data which we represent as the 11 component vector $z$. We do this by linking both $\boldsymbol{f}(\boldsymbol{x})$ and $\boldsymbol{z}$ to the actual system (in this case the real Universe) represented by $\boldsymbol{y}$, taking into consideration the Structural Uncertainty. In a rigorous Bayesian approach, this step is key in order to justify any further uncertainty statements; it is, however, a relatively unfamiliar process to many scientists. We employ a description that is widely used in computer modelling studies (e.g. Craig 1996; Kennedy \& O'Hagan 2001; Goldstein \& Rougier 2009). This involves the notion that when we evaluate GALFORM at the actual system properties, $\boldsymbol{x}^{*}$ say, then we aim to reproduce the actual system behaviour $\boldsymbol{y}$. This does not mean that we would expect perfect agreement between $\boldsymbol{f}\left(\boldsymbol{x}^{*}\right)$ and $\boldsymbol{y}$. Although GALFORM is a highly sophisticated simulator, it still offers a necessarily simplified account of the evolution of galaxies, and involves various numerical approximations. The simplest way to view the difference between $f^{*}=f\left(x^{*}\right)$ and $\boldsymbol{y}$ is to express this as

$\boldsymbol{y}=\boldsymbol{f}^{*}+\boldsymbol{\epsilon}_{\mathrm{md}}$,

where we consider the 11-vector $\boldsymbol{\epsilon}_{\mathrm{md}}$ as a random variable uncorrelated with $\boldsymbol{f}^{*}$. The 'Model Discrepancy' term $\boldsymbol{\epsilon}_{\mathrm{md}}$ represents the Structural Uncertainty. It comes from our judgments regarding the accuracy of the model and determines how close a fit between model output, $\boldsymbol{f}^{*}$, and an observation of $\boldsymbol{y}$ we require for an acceptable level of consistency between theory and observation.

As GALFORM is an approximation to the physical processes that occur during galaxy formation in the real Universe, we must acknowledge and attempt to quantify the level of this approximation, represented by $\boldsymbol{\epsilon}_{\mathrm{md}}$, in order for further analysis to be meaningful. While this is a difficult task, ignoring the model discrepancy will lead to all future statements being conditional on the current version of GALFORM being a perfect model of the Universe. Since we know that this is not the case, it is essential that we build in some degree of fuzziness into the comparison between the model and data. Failure to do so may result in us prematurely rejecting regions of parameter space in which a solution of interest resides. As the level of approximation in the model is reduced (by considering an improved version of the model say), $\boldsymbol{\epsilon}_{\mathrm{md}}$ will become smaller. In principle, this process of model improvement can be built into our statistical emulation so that we use our knowledge of previous versions of the GALFORM code both to speed up parameter exploration in newer versions and to obtain more realistic representations of the Structural Uncertainty (Goldstein \& Rougier 2009); however, we have not explored this possibility here.

As we are employing a Bayes Linear approach we only need to specify expectations and variances for $\boldsymbol{\epsilon}_{\mathrm{md}}$ : we give a summary of this process here, the full details of which can be found in Vernon et al. (2010).

We decompose $\boldsymbol{\epsilon}_{\mathrm{md}}$ into three uncorrelated contributions, each of which are 11-vectors that are assumed to have zero expectation:

$\boldsymbol{\epsilon}_{\mathrm{md}}=\boldsymbol{\Phi}_{\mathrm{IA}}+\boldsymbol{\Phi}_{\mathrm{DM}}+\boldsymbol{\Phi}_{E}$.

Here $\boldsymbol{\Phi}_{\mathrm{IA}}$ represents the discrepancy due to the eight inactive parameters that we did not model in detail in the initial waves of the analysis (i.e. the parameters that do not feature in Table 3). We assessed $\operatorname{Var}\left(\boldsymbol{\Phi}_{\mathrm{IA}}\right)$ from a small set of runs over the $8 \mathrm{D}$ inactive parameter space and found for $i=1, \ldots, 11$ that $0.015^{2}<\operatorname{Var}\left(\Phi_{\mathrm{IA}, i}\right)<0.32^{2}$. In later waves, we performed runs across all 16 inputs and hence the $\boldsymbol{\Phi}_{\text {IA }}$ term was then set to zero as this model discrepancy was now absorbed directly into the emulator.

$\boldsymbol{\Phi}_{\mathrm{DM}}$ is the discrepancy due to the finite number (40) of subvolumes used for the model runs: $\operatorname{Var}\left(\boldsymbol{\Phi}_{\mathrm{DM}}\right)$ was found by analysing the sample variance of the 1000 Wave 1 runs across sub-volumes, and it was estimated for $i=1, \ldots, 11$ that $0.014^{2}<\operatorname{Var}\left(\Phi_{\mathrm{DM}, i}\right)<$ $0.022^{2}$. For a sub-set of 100 runs, we confirmed this estimate by drawing a random set of 40 sub-volumes.

$\boldsymbol{\Phi}_{E}$ summarizes the structural deficiencies of the full GALFORM model itself, and is the component derived from subjective judgments regarding model accuracy. We proceeded by generating a random test set of luminosity functions by perturbing the observational data and smoothly interpolating. These were then compared to the observational data using an interactive tool which asked the expert user to judge whether to reject the corresponding region of parameter space on the basis of the comparison and previous experience of improvements to the GALFORM code and changes in cosmological models. Summarizing these tests, we concluded that a credible interval around the observational data, within which runs would be deemed acceptable would be approximately a factor of 2 wide in terms of galaxy counts, and hence $\pm \frac{1}{2} \log _{10}(2)$ on the $\log$ scale used throughout this paper (see e.g. Fig. 2). Relating this to a $\pm 2 \sigma$ interval (a conservative choice) this leads to the variance of each of the 11 components of $\Phi_{E}$ being assigned values: $\operatorname{Var}\left(\Phi_{E, i}\right)=\left(\log _{10}(2) / 4\right)^{2}=0.0753^{2}$ for $i=1, \ldots, 11$. Reassuringly, this results in the Bow06 model being close to the boundary of acceptable solutions. Examples of the range of fits that are deemed acceptable are illustrated in Fig. 8. The expectation was again set to $\mathrm{E}\left(\Phi_{E, i}\right)=0$, as it was thought that there were no significant asymmetries concerning this component of the Model Discrepancy. It is important to realize that while this assessment for $\boldsymbol{\Phi}_{E}$ is necessarily subjective, it was also chosen to be deliberately conservative. Once the volume of acceptable inputs has been identified 
corresponding to all uncertainties discussed in this section, it is then possible to explore the effect of reducing the size of $\operatorname{Var}\left(\boldsymbol{\Phi}_{E}\right)$.

Finally, we must make allowance for the uncertainty in observational measurements. Since, we cannot observe the system $\boldsymbol{y}$ (i.e. the actual Universe) without measurement error, we link it to the observations $z$ by

$z=\boldsymbol{y}+\boldsymbol{\epsilon}_{\mathrm{obs}}$,

where $\boldsymbol{\epsilon}_{\mathrm{obs}}$ is again a random quantity that represents the observational errors. It has expectation zero, and variance composed of contributions from the luminosity calibration uncertainty, the normalization uncertainty, $k+e$ errors and Poisson errors (see Norberg et al. 2002 for a discussion of how these terms are estimated). Fig. 2 shows the $2 \sigma$ error bars formed from the combination of all components of $\operatorname{Var}\left(\boldsymbol{\epsilon}_{\mathrm{md}}\right)$ and $\operatorname{Var}\left(\boldsymbol{\epsilon}_{\mathrm{obs}}\right)$. It should be noted that in most cases the Model Discrepancy terms dominate over the observational errors. (Fig. 5 shows the same error bars minus the $\boldsymbol{\Phi}_{\mathrm{IA}}$ component which is no longer relevant, as by Wave 4 we have modelled the effect of the remaining inactive variables within the emulator directly.)

With this structure linking $\boldsymbol{f}(\boldsymbol{x}), \boldsymbol{y}$ and $\boldsymbol{z}$ in place we can now proceed to learn about acceptable values of $\boldsymbol{x}$.

\subsection{Implausibility measures}

We want to learn about which values of the input parameters $x$ are likely to give an acceptable match between model output and observational data. We do this through use of an Implausibility Measure $I(\boldsymbol{x})$ defined over the input space. The Implausibility Measure describes the magnitude of the difference between the expected value of the GALFORM outputs and the observational data, standardized with respect to all relevant uncertainties. The basic idea is that for a particular value of $\boldsymbol{x}$, if $I(\boldsymbol{x})$ is large then we can discard this value of $\boldsymbol{x}$ as it is highly unlikely to yield a good match between model output and the observational data.

Using the emulator, the model discrepancy and the measurement errors we define the Univariate Implausibility Measure, at any input parameter point $\boldsymbol{x}$, for each component $i$ of the computer model $\boldsymbol{f}(\boldsymbol{x})$ as

$I_{(i)}^{2}(\boldsymbol{x})=\left|\mathrm{E}_{D_{i}}\left(f_{i}(\boldsymbol{x})\right)-z_{i}\right|^{2} / \operatorname{Var}_{D_{i}}\left(\mathrm{E}_{D_{i}}\left(f_{i}(\boldsymbol{x})\right)-z_{i}\right)$

where $\mathrm{E}_{D_{i}}\left(f_{i}(\boldsymbol{x})\right)$ and $\operatorname{Var}_{D_{i}}\left(f_{i}(\boldsymbol{x})\right)$ are the emulator expectation and variance adjusted by $D_{i}$ and $z_{i}$ is the observed data for component $i$. Introducing the model discrepancy and observational error terms, this can be re-written as

$I_{(i)}^{2}(\boldsymbol{x})=\frac{\left|\mathrm{E}_{D_{i}}\left(f_{i}(\boldsymbol{x})\right)-z_{i}\right|^{2}}{\left(\operatorname{Var}_{D_{i}}\left(f_{i}(\boldsymbol{x})\right)+\operatorname{Var}\left(\epsilon_{\mathrm{md}, i}\right)+\operatorname{Var}\left(\epsilon_{\mathrm{obs}, \mathrm{i}}\right)\right)}$

where $\operatorname{Var}\left(\epsilon_{\mathrm{md}, i}\right)$ and $\operatorname{Var}\left(\epsilon_{\mathrm{obs}, i}\right)$ are the (univariate) Model Discrepancy variance and Observational Error variance.

When $I_{(i)}(\boldsymbol{x})$ is large this implies that, even given all the uncertainties present in the problem, we would be unlikely to obtain a good match between model output and observed data where we to run the model at input $\boldsymbol{x}$. This means that we can cut down the input space by imposing suitable cut-offs on the implausibility function (a process referred to as History Matching). Regarding the size of $I_{(i)}(x)$, if we assume that for fixed $\boldsymbol{x}$ the appropriate distribution of $\left(f_{i}\left(\boldsymbol{x}^{*}\right)-z_{i}\right)$ is both unimodal and continuous, then we can use the $3 \sigma$ rule which implies that if $\boldsymbol{x}=\boldsymbol{x}^{*}$, then $I_{(i)}(\boldsymbol{x})<3$ with a probability of approximately 0.95 . This is a powerful result that applies to any distribution that is unimodal and continuous, even if it is asymmetric. It suggests that values higher than 3 would imply that the point $\boldsymbol{x}$ should be discarded. This is still a very conservative bound: we would expect the distribution of $\left(f_{i}\left(x^{*}\right)-z_{i}\right)$ to be somewhat better behaved and hence choose slightly tighter bounds, as discussed in Section 3.8.

It should be noted that since the implausibility relies purely on means and variances (and therefore can be evaluated using the Bayes Linear methodology), it is both tractable to calculate and simple to use to reduce the input space.

One way to combine these univariate implausibilities is by maximizing over outputs:

$I_{M}(\boldsymbol{x})=\max _{i} I_{(i)}(\boldsymbol{x})$.

We can similarly define $I_{2 M}(\boldsymbol{x})$ and $I_{3 M}(\boldsymbol{x})$ to be the second and third highest of the 11 univariate Implausibility Measures at the point $\boldsymbol{x}$. These are clearly more conservative measures since a model will not be deemed implausible on the basis of a single bin.

If we construct both a multivariate emulator and multivariate model discrepancy (as is described in detail in Vernon et al. 2010), then we can define the corresponding multivariate Implausibility Measure:

$I_{\mathrm{MV}}^{2}(\boldsymbol{x})=\left(\mathrm{E}_{D}(\boldsymbol{f}(\boldsymbol{x}))-\boldsymbol{z}\right)^{T} \operatorname{Var}_{D}\left(\mathrm{E}_{D}(\boldsymbol{f}(\boldsymbol{x}))-\boldsymbol{z}\right)^{-1}\left(\mathrm{E}_{D}(\boldsymbol{f}(\boldsymbol{x}))-\boldsymbol{z}\right)$,

which becomes

$I_{\mathrm{MV}}^{2}(\boldsymbol{x})=\left(\mathrm{E}_{D}(\boldsymbol{f}(\boldsymbol{x}))-\boldsymbol{z}\right)^{T}$

$\times\left(\operatorname{Var}_{D}(\boldsymbol{f}(\boldsymbol{x}))+\operatorname{Var}\left(\boldsymbol{\epsilon}_{\mathrm{md}}\right)+\operatorname{Var}\left(\boldsymbol{\epsilon}_{\mathrm{obs}}\right)\right)^{-1}\left(\mathrm{E}_{D}(\boldsymbol{f}(\boldsymbol{x}))-\boldsymbol{z}\right)$

where $\boldsymbol{f}(\boldsymbol{x})$ is the full 11-vector model output and $\operatorname{Var}_{D}(\boldsymbol{f}(\boldsymbol{x})), \operatorname{Var}\left(\boldsymbol{\epsilon}_{\mathrm{md}}\right)$ and $\operatorname{Var}\left(\boldsymbol{\epsilon}_{\mathrm{obs}}\right)$ are all $11 \times 11$ covariance matrices. Again, large values of $I_{\mathrm{MV}}(\boldsymbol{x})$ imply that we would be unlikely to obtain a good match between model output and observed data where we run the model at input $\boldsymbol{x}$. Choosing a cut-off for $I_{\mathrm{MV}}(\boldsymbol{x})$ is more complicated. As a simple heuristic, we might choose to compare $I_{\mathrm{MV}}(\boldsymbol{x})$ with the upper critical value of a $\chi^{2}$ distribution with degrees of freedom equal to the number of outputs. For further discussion of Implausibility Measures, see Vernon et al. (2010).

\subsection{History matching via implausibility}

History Matching is the process of identifying the set $\mathcal{X}^{*}$ of all possible values of $\boldsymbol{x}^{*}$, that is the set of points that would give acceptable matches between model output and observational data. Identifying $\mathcal{X}^{*}$ is a difficult task as often $\mathcal{X}^{*}$ represents a complicated object in a high-dimensional space. $\mathcal{X}^{*}$ could also comprise disconnected volumes, which could even possess non-trivial topology. In many applications $\mathcal{X}^{*}$ occupies an extremely small fraction of the original input space, with large volumes of input space leading to very poor matches to the observed data.

We employ an iterative technique where the Implausibility Measures are used to perform the History Matching process. The basic strategy is based around discarding values of $\boldsymbol{x}$ that are highly unlikely to yield acceptable matches between model output and observational data. This is done by applying a cut-off on the Implausibility Measures defined in Section 3.7. As the Implausibility Measures are constructed using the emulator, they are fast to evaluate and therefore we can efficiently identify values of $\boldsymbol{x}$ that will be discarded, for example, in Wave 1 we discard all values of $\boldsymbol{x}$ that do not satisfy both:

$I_{2 M}(\boldsymbol{x})<I_{2 \text { cut }}$ and $I_{3 M}(\boldsymbol{x})<I_{3 \text { cut }}$ 
Table 4. The fraction of parameter space considered acceptable in each wave of emulation. Column 1: the wave; Column 2, the number of model runs used to construct the emulator; Column 3 , the number of active variables; Columns 4-7, the implausibility cut-off threshold; Column 8, the fraction of the parameter space estimated to be acceptable. Note that in Wave 5 we do not construct an emulator, but we impose a cut-off of $I_{M}<2.5$ on the Wave 5 runs to generate the 113 acceptable runs used in Section 5.

\begin{tabular}{cccccccc}
\hline Wave & Runs & \#Act. & $I_{\text {cut }}$ & $I_{2 \text { cut }}$ & $I_{3 \text { cut }}$ & $I_{M V \text { cut }}$ & $\begin{array}{c}\text { fraction of } \\
\text { space (per cent) }\end{array}$ \\
\hline 1 & 1000 & 5 & - & 2.7 & 2.3 & - & 14.9 \\
2 & 1400 & 8 & - & 2.7 & 2.3 & - & 5.9 \\
3 & 1600 & 8 & - & 2.7 & 2.3 & 26.75 & 1.6 \\
4 & 2000 & 10 & 3.2 & 2.7 & 2.3 & 26.75 & 0.26 \\
5 & 2000 & - & 2.5 & - & - & - & $(0.014)$ \\
\hline
\end{tabular}

where $I_{2 M}(\boldsymbol{x})$ and $I_{3 M}(\boldsymbol{x})$ are the second and third highest univariate Implausibility Measures defined in Section 3.7 and $I_{2 \text { cut }}$ and $I_{3 \text { cut }}$ are the corresponding implausibility cut-offs. Table 4 shows all the Implausibility Measures used in each of the waves along with the corresponding cut-offs. Note that in early waves we make the conservative choice of using only $I_{2 M}(\boldsymbol{x})$ and $I_{3 M}(\boldsymbol{x})$ (and not $I_{M}(\boldsymbol{x})$ ), so that the cut-off we impose is not sensitive to the possible failings of an individual emulator point on the luminosity function. This allows slightly tighter cuts to be chosen for $I_{2 \text { cut }}$ and $I_{3 \text { cut }}$ as is shown in Table 4.

Equation (25) defines a volume of input space that we refer to as non-implausible and denote as $\mathcal{X}_{1}$. This non-implausible volume should hopefully contain the set $\mathcal{X}^{*}$, that is $\mathcal{X}^{*} \subset \mathcal{X}_{1}$. In the first wave of the analysis which we are describing here, $\mathcal{X}_{1}$ will be substantially larger than $\mathcal{X}^{*}$. This is because it will contain many values of $\boldsymbol{x}$ that only satisfy the implausibility cut-off given by equation (25) because of a substantial emulator variance $\operatorname{Var}(\boldsymbol{f}(\boldsymbol{x}))$. If the emulator had a high degree of accuracy over the whole of the input space so that $\operatorname{Var}(\boldsymbol{f}(\boldsymbol{x}))$ was small compared to the Model Discrepancy and the Observational Error variances, then the non-implausible volume defined by $\mathcal{X}_{1}$ would be comparable to $\mathcal{X}^{*}$ and the History Match would be complete. However, to construct such an accurate emulator for any realistic computer model (and especially for GALFORM) would require an infeasible number of runs of the model. Even if such a large number of runs were possible it would be an extremely inefficient method: we do not need the emulator to be highly accurate in regions of the input space where the outputs of the model are clearly very different from the observed data.

This is the main motivation for our iterative approach. In each wave we design a set of runs over the current non-implausible volume denoted as $\mathcal{X}_{i}$, emulate using these runs, calculate the Implausibility Measure and impose a cut-off to define a new (smaller) non-implausible volume denoted as $\mathcal{X}_{i+1}$ which should satisfy $\mathcal{X}^{*} \subset \mathcal{X}_{i+1} \subset \mathcal{X}_{i}$. As we progress through each iteration the emulator at each wave will become more and more accurate, but will only be defined over the previous non-implausible volume defined by the previous wave's implausibility.

As we proceed through waves of emulation the volume being emulated decreases, and the GALFORM function should become smoother over the restricted range of interest. As a result, it becomes easier to capture more of the behaviour using the regression terms in the emulator (i.e. by fitting a cubic polynomial to the GALFORM output). Furthermore, the density of runs that inform us about the models' behaviour increases and the Gaussian Process part of the emula- tor becomes more accurate. As the output of the runs have been restricted and the effects of certain dominant variables limited, it becomes easier to identify additional active variables which are then used in both the regression and the Gaussian Process terms, further increasing the accuracy of the emulation.

This iterative process is continued until the emulator variance is smaller than the model discrepancy variance and observational error variance. We have completed four iterations or Waves in this analysis, and the subsequent results in this paper are from Wave 4.

\subsection{Projection pursuit}

The end result of the emulator analysis is to identify a region of parameter space in which models produce an acceptable fit to the $b_{\mathrm{J}}$ - and $K$-band luminosity functions. However, comprehending the resulting space is rather challenging. As we will show, while the parameter space of acceptable model occupies only a small fraction of the overall parameter space, acceptable fits can be found over a wide range of input parameters. This situation arises because the acceptable space takes the form of a thin curved hypersurface.

The aim of projection pursuit is to select a suitable co-ordinate system that allows the geometry of the acceptable region to be better understood. We achieve this using PCA (e.g. Jolliffe 2002; Zito et al. 2009). However, in contrast to many applications of PCA, we are primarily concerned with the components with smallest variance. These components define an optimal set of projections for displaying the data, and the relation between the PCA vectors and the input parameters. The latter connection has the potential to inform us about the physics of galaxy formation.

\subsection{Exploring constraints from additional data sets}

We have adopted a strategy in which the primary calibration of our model comes from the local $b_{\mathrm{J}}$ - and $K$-band luminosity functions. Nevertheless, we wish briefly to explore whether adding additional data sets would impose further constraints on the range of acceptable model parameters. In this paper, we do not aim to make an exhaustive exploration of the possible data sets and limit our attention to just a small fraction of the possible local data. We use a simple $\chi^{2}$ statistic to assess the relative performance of models in these additional tests and ask about the region of parameter space which matches the additional data at a similar level of performance to Bow06 (as well as adequately matching the observed luminosity functions). As we show in Section 5.2, the model experiences contradictory pressures from the observed disc sizes and the normalization of the TF relation, possibly indicating that a revised treatment of angular momentum is required in the Bow06 version of the GALFORM code. This clearly illustrates the need to carefully define the model discrepancy terms for these additional data sets before they are used to exclude regions of parameter space. We present further exploration of additional data sets in a future paper (Benson \& Bower 2010).

\section{RESULTS}

The results described below were obtained with four waves of emulation. The implausibility cut-off threshold for each wave is shown in Table 4, together with the fraction of the parameter space considered acceptable after the emulator has been constructed. The table also gives the number of runs used during each wave.

After four waves of emulation, the uncertainties in the emulator are small and the implausibility of each run is becoming dominated 
by the intrinsic model discrepancy $\boldsymbol{\epsilon}_{\mathrm{md}}$. Uncertainties in the observational measurement of the luminosity function make almost negligible contribution, and the dominant contribution to the model uncertainty comes from the model discrepancy term, $\boldsymbol{\Phi}_{E}$ (see discussion in Section 3.6).

At this point, the emulator suggests that only 0.26 per cent of the initial parameter volume is 'not implausible'. While this volume is small, we will show below that an 'acceptable' fit can be obtained with a wide range of values for some parameters. Note that we are being careful in our use of language here. We do not know for certain what the outcome of running the model will be at a particular set of parameter values, except close to values at which we have already performed a model run. We do, however, have a prediction for the expectation and variance. Fig. 6 shows a comparison of the expectation of the emulator and its uncertainty with the results of actual model runs and we discuss this comparison further in Section 4.2. However, as we should expect, only a fraction of the runs within the 'not implausible' region actually result in sufficiently good fits to the luminosity function to be considered 'acceptable'. There are two factors involved here. First, we are tightening the required implausibility from 3.2 to 2.5 . In 16 dimensions, this results in a large reduction in the surface of the interesting region. Secondly, for many runs the expectation of the emulator is that the model implausibility lies above 2.5 , but the residual emulator variance cannot rule out the region as unacceptable without direct evaluation. This uncertainty arises from emulator variance, and not from observational error or model discrepancy terms.

\subsection{Emulating the luminosity function}

A serious problem for such high-dimensional parameter sets is to find a way of representing the implausibility map. Projecting the full $10 \mathrm{D}$ map (of active variables) down to two dimensions so that it can be printed loses considerable information. We can try to compensate for this by showing the full set of projections as a matrix (this is commonly referred to as a 'pairs plot'.). Fig. 4 shows the implausibility space projected on to pairs of parameters in this way. Note that only active variables are shown so that there are 45 plots of 10 variable pairs. The parameters have been scaled to range over \pm 1 using the initial range given in Table 1 .

Plots below the diagonal show the projected minimum implausibility surface. The colour code is set so that green indicates that the region is 'not implausible'. The implausible region is shown in red. The minimum implausibility is determined by evaluating the emulator over a grid of values for the two 'visible' parameters and a Latin hypercube of parameters in the unseen variables. Because the hypervolume of acceptable solutions is very thin in some projections, a large number of evaluations are required in order to obtain a reliable projection of the minimum value. Even though each evaluation is almost $10^{7}$ times quicker than performing a GALFORM model evaluation, this means that such plots cannot be made interactively.

It is immediately apparent that many of the projections contain a substantial fraction of green covering a large fraction of the parameter space. For example, the $\alpha_{\text {cool }}$ parameter was allowed to vary in the range 0.1 to 1.2 , and it is not implausible to find acceptable fits throughout this region. This is the result of projecting over a large number of hidden parameters, however, and it is apparent that varying the visible parameters had physical effects that can be compensated for by variations in the other parameters. In order to better appreciate the underlying geometry, therefore, it is helpful to plot the 'optical depth' of the projected hypervolume. Therefore above the diagonal, we show the fraction of the evaluations (for each pair of fixed visible parameters) that resulted in low implausibility values. This allows the viewer to distinguish regions that have a low minimum implausibility but require very precise coordination of the unseen parameters, from regions in which a low implausibility is obtained for a wide range of the unseen parameters. One has the intuitive sense that the 'best' solution lies in a region that has a large depth, but this assertion does not necessarily hold. Thus, the appearance of regions in this optical depth plot needs careful interpretation. For reference, the Bow06 model is shown by a black point. It is often centred in a 'deep' region of low implausibility, but in some projections it is offset from the depth-weighted centre of the region. It is apparent from this that the Bow06 is not a 'typical' model that matches the zero redshift luminosity function: illustrating the limitations of searching parameter space to find a single acceptable model.

Higher dimensional projections can also be used to reveal more of the underlying structure. The typical hypersurface geometry is that of a thin, slightly curved plane. For a few variables, it is nevertheless informative to look at the range of the 'not implausible' region in a 1D sense, and this is given in Table 5. While this region covers a large fraction of the initial range for many parameters, a few of the parameters are significantly constrained. For example, the emulator shows that it is implausible that runs with low values of $V_{\text {hot,disc }}$ (below $300 \mathrm{~km} \mathrm{~s}^{-1}$ ) will result in acceptable fits to the luminosity functions. Similarly, the emulator suggests that the disc stability parameter cannot be reduced below 0.73 , showing that disc instabilities are a key component of the model and that the role of instabilities cannot be replaced by altering the sensitivity of galaxies to high-mass ratio mergers (Parry, Eke \& Frenk 2009).

The parameters that are shown as inactive in Table 1 have no clear effect on the luminosity function and are treated as an additional source of uncertainty in the emulator. Runs close to the implausibility cut-off may result in acceptable fits to the luminosity functions if these parameters are carefully chosen. However, our procedure is conservative and this is taken into account in deciding whether a region is implausible or not. However, while the emulator can identify regions for which an acceptable fit is implausible, it does not guarantee that a run in the remaining region will actually result in a good fit to the luminosity function. This is a consequence of our conservative approach: the 'not implausible' region has a cut-off threshold of $I_{M}<3.2$, while we only deem a model to be 'acceptable' if $I_{M}<2.5$. Therefore, in order to demonstrate that an acceptable match to the luminosity function can be obtained, we must perform a model run at the point in question.

\subsection{Comparing the emulator with model runs}

In the previous section, we illustrated the shape of the low implausibility region in parameter space. It is important to stress that this is a region in which good fits might be obtained, but that an acceptable match is not guaranteed. To proceed, we investigate the success of the emulator technique by randomly generating 2000 model runs (which we refer to as 'Wave 5') with various parameter values within the not implausible region of Wave 4. Our aim is twofold. First, we wish to show that the model runs do indeed return matches to the luminosity function within the expected uncertainties, and that good descriptions of the luminosity function are indeed found throughout the range of parameter space illustrated in Fig. 4. Secondly, we wish to identify a set of model runs that give acceptable matches to the observed luminosity functions, and that can be used as the basis for our exploration of the constraints imposed by additional data sets. 


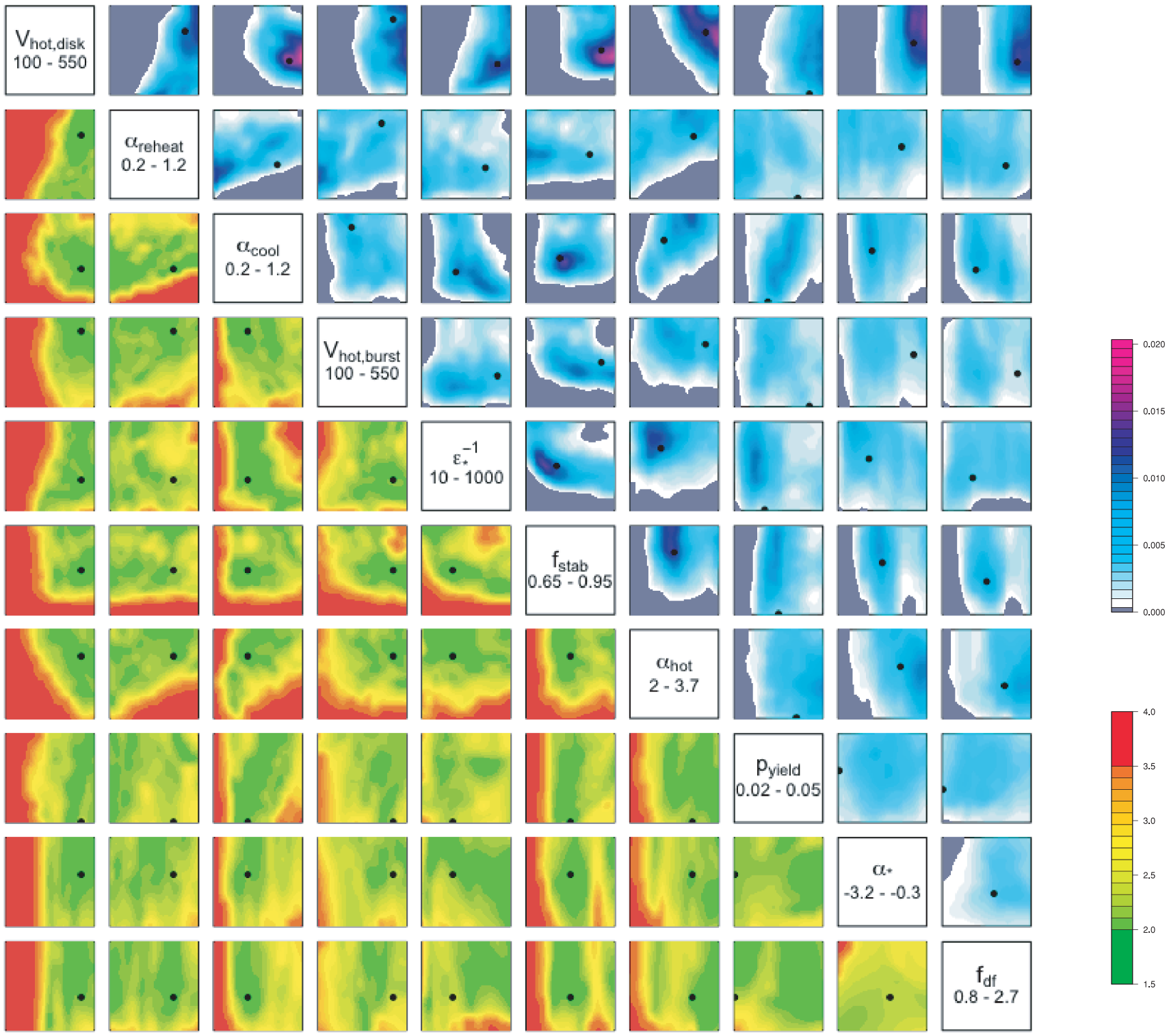

Figure 4. 2D projections of the implausibility landscape of the Wave 4 emulator for some of the major parameters. Plots below the diagonal show the projected minimum implausibility predicted by the Wave 4 emulator. We sample data points in the hidden dimensions using a Latin hypercube design, accumulating the minimum implausibility and the fraction of points lying below the implausibility cut-off (see Table 4). The emulator suggests that green regions are likely to give acceptable fits to the luminosity function data, for some choice of the hidden parameters (given our model discrepancy). In the red region the emulator confidently suggests that acceptable matches are implausible, regardless of the values of the hidden parameters. For comparison, the Bow06 model is shown as a black point. Plots above the diagonal give an impression of the line-of-sight depth of the acceptable region with blue/purple regions indicating that a high fraction of the 'not implausible' points are aligned at this projected position. Note that plots above the diagonal have $x$ and $y$ axes transposed to make comparison with the maximum implausibility plots more apparent. Although it seems that only a small region of the projected space is ruled out as implausible, the projected space frequently has a very thin, but extended, geometry.

Since we are now comparing genuine model evaluations with the data, the emulation stage is no longer required and the emulator variance term, $\operatorname{Var}_{D_{i}}\left(f_{i}(\boldsymbol{x})\right)$ in equation (25), is replaced by a small factor based on the stochastic variation in model evaluations. We denote this revised Implausibility Measure $I_{M}^{\prime}$. The denominator in $I_{M}^{\prime}$ thus includes only contributions from the model discrepancy, observational errors and the repeatability of model runs. Because we are now referring to actual model evaluations, we can refer to a model as 'acceptable' rather than 'not implausible'. We identify 'acceptable' models as those for which $I_{M}^{\prime}<2.5$. It is important to note that we distinguish acceptable models by a lower implausi- bility cut than the threshold used to reject implausible regions (see Table 4).

The luminosity functions of the Wave 5 model runs are shown in Fig. 5. The lines have been colour code to reflect the implausibility derived for the run using the colour scale of Fig. 4. Note that the $2 \sigma$ error bars shown on the observational points include the effect of the model discrepancy and repeatability. Around the knee of the luminosity function the statistical errors on the luminosity function are small and are dominated by the model discrepancy term. Vertical lines show the points at which we have emulated the luminosity function. An acceptable luminosity function must pass close to 
Table 5. The parameter ranges of models which gave acceptable luminosity function matches. The inactive variables range over their full range given in Table 1.

\begin{tabular}{lcc}
\hline & Min & Max \\
\hline$\epsilon_{\star}^{-1}$ & 46 & 1000 \\
$\alpha_{\star}$ & -3.2 & -0.3 \\
$p_{\text {yield }}$ & 0.02 & 0.05 \\
$V_{\text {hot,disc }}$ & 300 & 550 \\
$V_{\text {hot, burst }}$ & 190 & 550 \\
$\alpha_{\text {hot }}$ & 2.3 & 3.7 \\
$\alpha_{\text {reheat }}$ & 0.2 & 1.2 \\
$\alpha_{\text {cool }}$ & 0.38 & 1.2 \\
$f_{\text {df }}$ & 0.8 & 2.7 \\
$f_{\text {stab }}$ & 0.73 & 0.95 \\
\hline
\end{tabular}
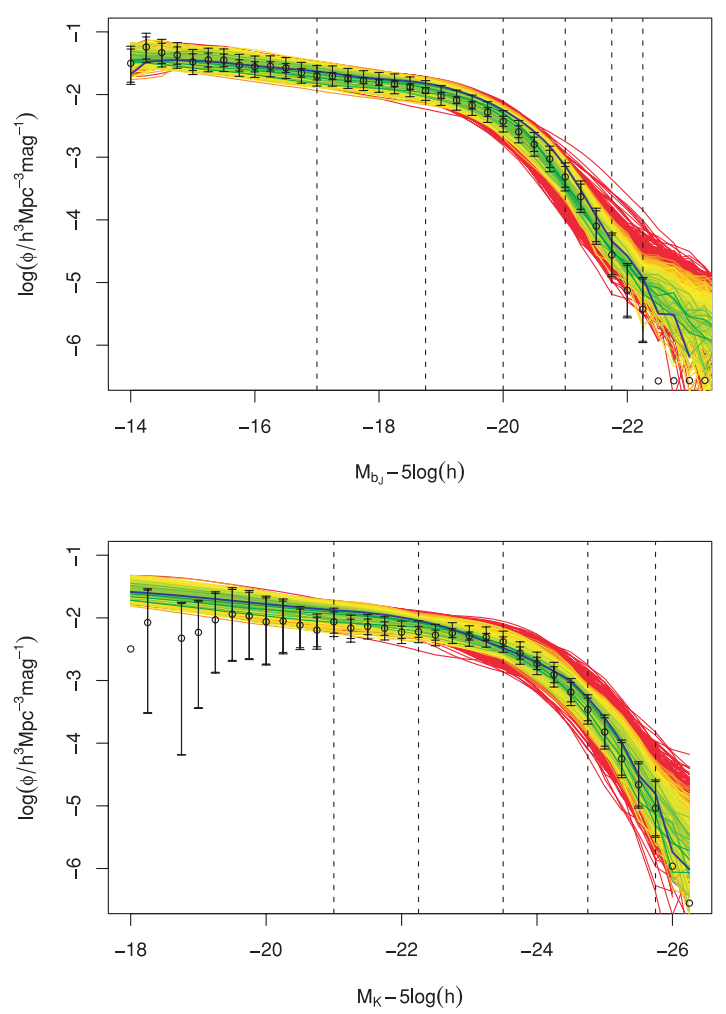

Figure 5. The $b_{\mathrm{J}}$ and $K$ luminosity functions of the model runs evaluated in Wave 5 . Runs are colour coded by the model run implausibility. For comparison, the Bow06 model is shown as a blue line. Note that $2 \sigma$ error bars are shown. The inner error bars include the contributions of observational errors and repeatability, but exclude the subjective $\boldsymbol{\Phi}_{E}$ term. Outer error bars also include the full model discrepancy term. Green curves are considered acceptable fits and are tested against other observational constraints in Section 4.3. Vertical lines show the magnitudes at which the model is compared to the observational data in order to determine its implausibility.

the error bars at the lines. As can be seen, this generally provides a good description of the shape of the luminosity function and justifies our assertion that the shape is well described by the 11 outputs chosen for emulation. In common with previous version of the code, acceptable models tend to lie above the lowest luminosity measurements. The distinction between green ('acceptable') and yellow ('not quite acceptable') models is that the latter tend to miss one or more data points. It is clear, however, that the points at the bright end of the luminosity function, particularly in the $b_{\mathrm{J}}$ band, present the greatest challenge for the galaxy formation model.

We can first use the model evaluations to confirm the success of the emulator in describing the luminosity function behaviour. In Fig. 6, we compare the values of the luminosity function in different absolute magnitude bins from direct evaluations of the model with predictions from the Wave 4 emulator. The panels are labelled by the absolute magnitude in either the $b_{\mathrm{J}}$ or $K$ band at which the luminosity function is sampled. The runs are shown in order of their emulator expectation, which is drawn as a think black line. Note that adjacent points are not adjacent in parameter space. The result of evaluating the model for each parameter set is shown as a blue circle. If the model evaluation agreed exactly with the expectation value, the blue points would lie perfectly on the line. However, we expect the points to scatter about the line as a result of the uncertainties in the emulation and the Monte Carlo nature of the model. The extreme of the predicted range of these uncertainties are shown as the red shaded region. This is formally a $3.2 \sigma$ deviation (although we do not necessarily expect the tails of the distribution to be Gaussian) that is used to define the not implausible region. Note that some regions of the luminosity function are easily emulated, and the points lie close to the expected value. In other regions, particularly at the bright end of the $b_{\mathrm{J}}$ luminosity function, there is significantly greater variance. This is telling us that it is hard to precisely emulate the behaviour of GALFORM in these regions. The uncertainty is, however, well described by the emulator's predicted uncertainty. The ability of the emulator to capture this variance is key since it has allowed us to efficiently cut down the full parameter space of models. Note that a series of similar diagnostics were performed for each emulator at each wave of the analysis, and in each case the emulator was found to provide the expected levels of accuracy (see Vernon et al. 2010 for details).

Since we are confident that the emulator has successfully directed the selection of Wave 5 parameter sets, we continue to compare the parameter space of acceptable models with that suggested by the emulator. Fig. 7 shows a pairs plot of the Wave 5 evaluations. Each dot shows the implausibility of the model evaluation, colour coded to match the implausibility colour scale of Fig. 4. The points have been superposed so as to illustrate the most acceptable in each region. Acceptable luminosity function fits (green points) are found throughout the parameter space, and the figure bears striking similarity to the analogous Fig. 4, which was based on emulator predictions rather than model evaluations. This again confirms that the emulator successfully captures the behaviour of the GALFORM model.

Of the 2000 model evaluations, 113 resulted in acceptable models according to the criterion $I_{M}^{\prime}<2.5$. The reduction in volume is not surprising since we have tightened the implausibility threshold and eliminated the emulator variance. Furthermore, many of the evaluations were performed for marginal models for which the emulator gave only a small chance of an acceptable match (recall that the surface of the acceptable parameter space is huge in 10 dimensions). Despite this apparent inefficiency, if we wish to make a systematic investigation of parameter space it is important that marginal models are evaluated. Of course, with only a relatively small number of evaluations, some parameter space projections suffer from considerable shot noise, particularly the low 'optical depth' regions seen above the diagonal in Fig 4. Further evaluations of the model could be used to fill in these regions if required. It is already apparent, however, that acceptable fits to the luminosity functions can be found throughout the wide range of parameter values suggested by the emulator analysis. 

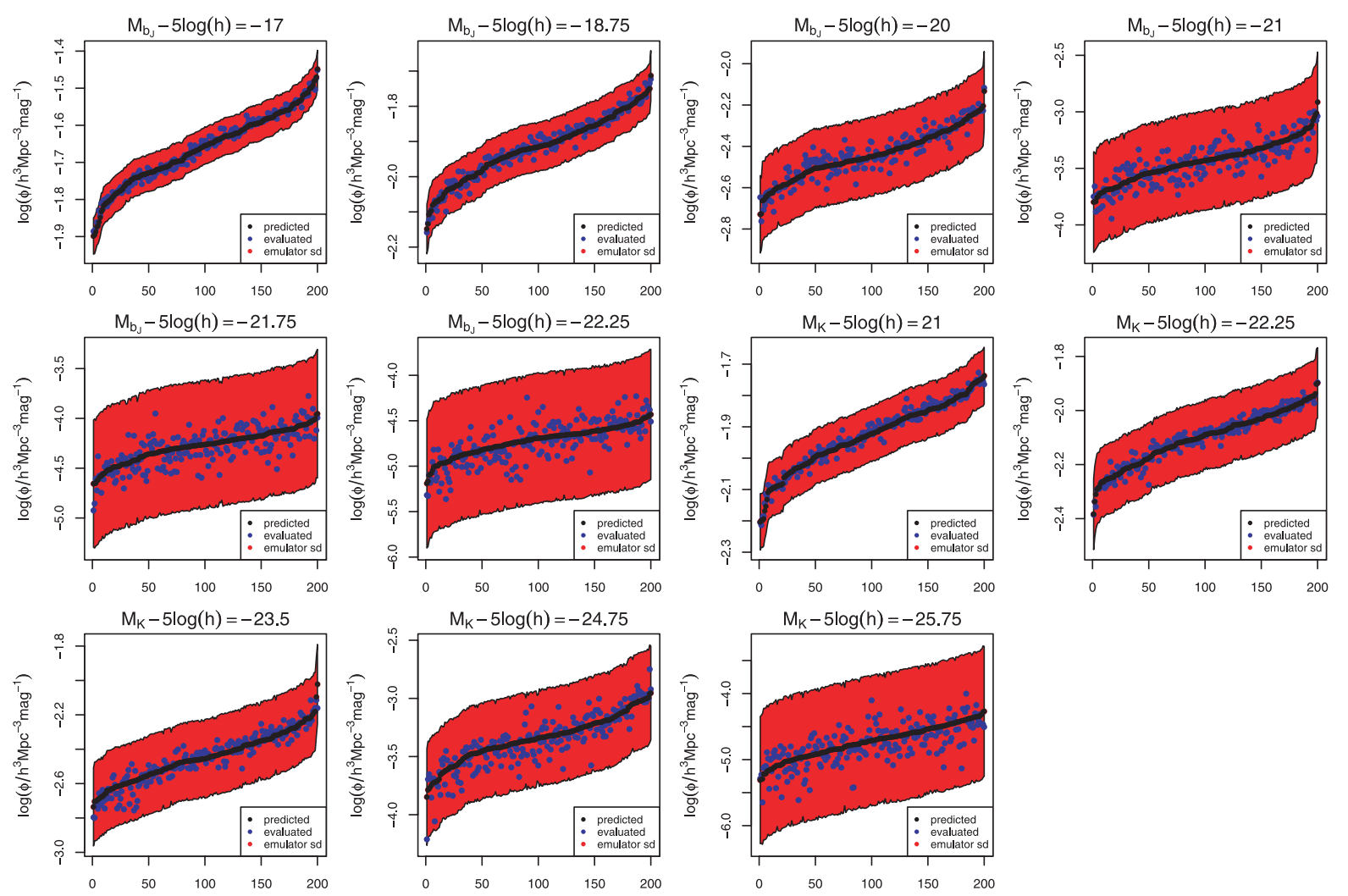

Figure 6. Values of the $b_{\mathrm{J}}$ - and $K$-band luminosity functions for direct model evaluations compared with the expectation and variance predicted by the emulator. The figure shows 200 runs selected at random from Wave 5. The panels are labelled with the relevant $b_{\mathrm{J}}$ or $K$ magnitude at which the luminosity function is sampled: these correspond to the vertical lines in Fig. 5. The $x$-axis shows the run number ordered by the value of the luminosity function predicted by the emulator. The $y$-axis shows the value of the luminosity function. The solid black line shows the expectation value of the emulator: because the runs have been ordered, the black line traces a smooth curve (but adjacent points need not be close in parameter space). The red shaded regions indicates the $3.2 \sigma$ range of uncertainty in the emulator output. The open circles show the values of model runs with each parameter set. Note that some regions of the luminosity function are easily emulated, while other regions, particularly the bright end of the $b_{\mathrm{J}}$ luminosity function, show significantly greater variance.

We conclude that the emulator method provides an accurate scheme for identifying model parameter sets that are very likely to yield acceptable fits to the luminosity functions. Before we examine the physical links between the parameters in the acceptable regions, we use the 113 acceptable runs to examine how well models which make acceptable fits to the luminosity functions perform in matching other low-redshift data sets.

\subsection{Further constraints from additional data}

The main focus of this paper is to investigate the constraints derived from the $b_{\mathrm{J}}$ and $K$ luminosity functions. This follows the methodology of Bow06. However, it is interesting to briefly examine how the model may be constrained by including additional data in the comparison. In order to make an initial investigation, we apply these constraints as a second phase, so that we only consider models which survive the primary criterion of generating an acceptable match to the luminosity functions, and we base the further exploration on the 113 fully acceptable models that were identified in the previous section. The luminosity functions derived from these models are shown in Fig. 8.

We now outline the additional data sets that we consider. Note that the first four physical properties listed below were already used by Cole et al. (2000) and Baugh et al. (2005) in choosing the parameter values in their respective versions of GALFORM, though we have here updated some of the observational data used. Further details of the data sets and our approach to the comparison are given in Benson \& Bower (2010).

(i) Disc size: we compare to disc size data from de Jong \& Lacey (2000). We compute the $\chi^{2}$ values in a series of bins in both magnitude and size. The ideal model would therefore match not only the sizes of local galaxies, but also the spread in size. The predicted disc size distribution depends strongly on the angular momentum of accreted gas which has a complex dependence on halo growth.

(ii) TF relation: Galaxy formation models have traditionally struggled to match the TF relation. The normalization and slope of the relation depend both on the relationship between stellar mass and halo mass and on the baryonic contraction of the halo. We compare with $i$-band data from Pizagno et al. (2007).

(iii) Gas metallicity: this is an important constraint on the effectiveness of supernova-driven feedback. We compare with data from Tremonti et al. (2004) on the oxygen abundance of gas in late-type galaxies in the SDSS.

(iv) Gas mass to $L_{B}$ : the cold gas reservoir is sensitive to the rate at which gas is accreted, the rate at which it is converted into stars and the effectiveness of supernova-driven feedback. We compare with a compilation of H I data from Huchtmeier \& Richter (1988) 


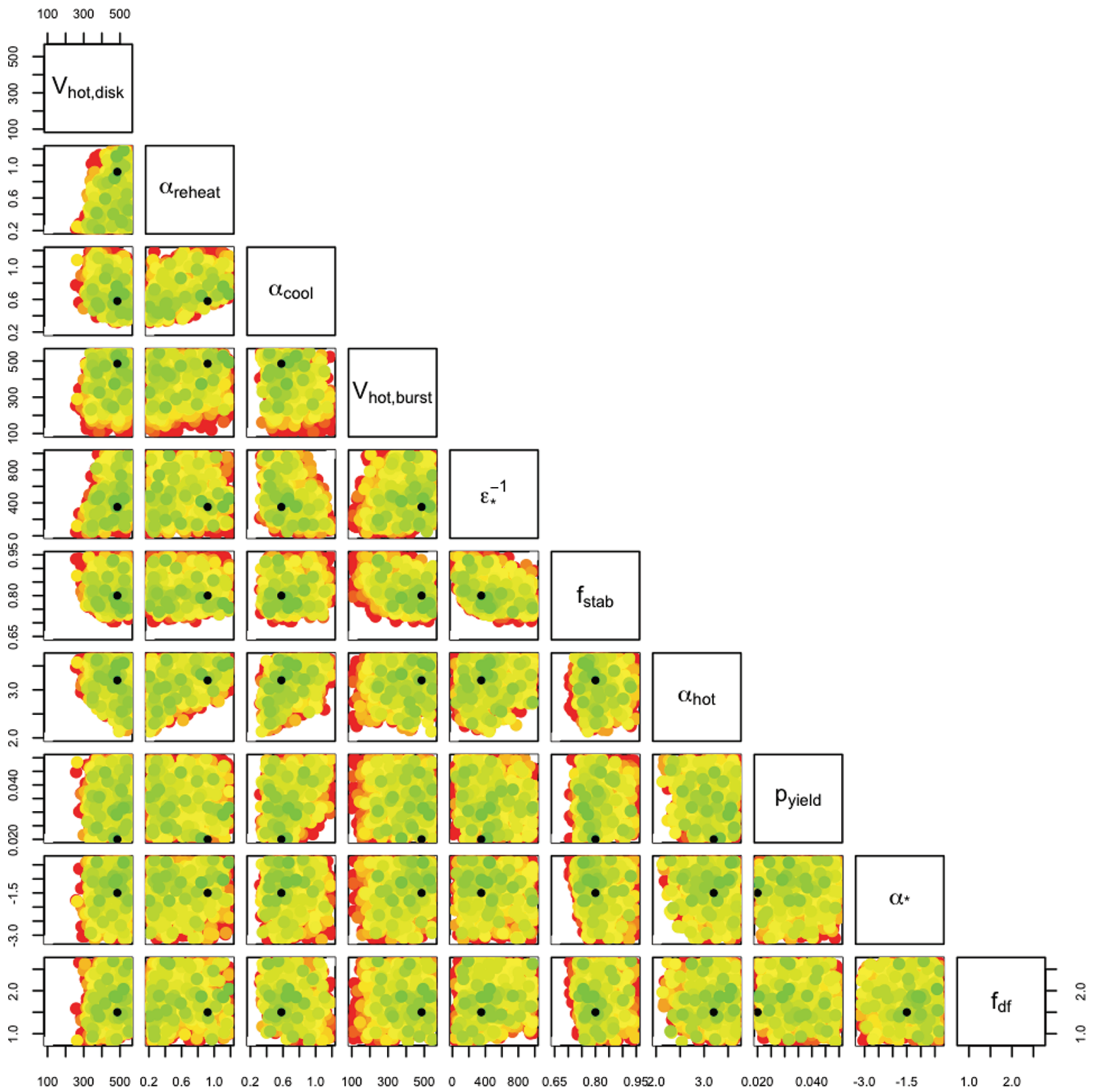

Figure 7. The Wave 5 model runs plotted on a set of 2D projections. The points are colour coded by the model run implausibility, rather than the emulator's predicted implausibility. Note that the model has only been evaluated in the 'not implausible' region defined by the Wave 4 emulator. Green points resulted in acceptable luminosity functions given the model variance and repeatability. The Bow06 model is shown as a black point.

by computing the mean and standard deviation of the ratio of the $\mathrm{H}_{\mathrm{I}}$ gas mass to $B$-band luminosity as a function of $B$-band magnitude. We only consider model galaxies with a bulge to total ratio of less than 0.4 and gas mass fraction greater than 3 per cent.

(v) SDSS colours: we compare with the overall distribution of galaxy $g-r$ colours from Weinmann et al. (2006). The vast amount of data available for this test results in a large number of bins. As discussed in Font et al. (2008), this match can be substantially improved by adjusting the stellar yield of the model.

(vi) BH mass: we compare to data from Häring \& Rix (2004). This is a weak test for our model since although the parameter $F_{\mathrm{bh}}$ has little effect on the luminosity function output, its value can be adjusted to fine tune the black hole mass normalization. Since the parameter is inactive, we could have made this adjustment without having discernible effect on the luminosity function. We show the comparison for completeness only, and we have not undertaken this fine tuning step.

For each additional data set, we reduce the comparison to a single $\chi^{2}$ value. Except where indicated, this is achieved by comparing the binned distributions of model and observed galaxies. The $\chi^{2}$ values for the Bow06 model and an indication of the number of bins used in each test is given in Table 6. Some examples of the fits to these additional data sets are shown in Fig. 9. Further examples are shown in Benson \& Bower (2010). As has been extensively discussed, the $\chi^{2}$ value gives only a coarse indication of the fit of the model to the data, and makes no allowance for model discrepancy terms that estimate the level at which we 

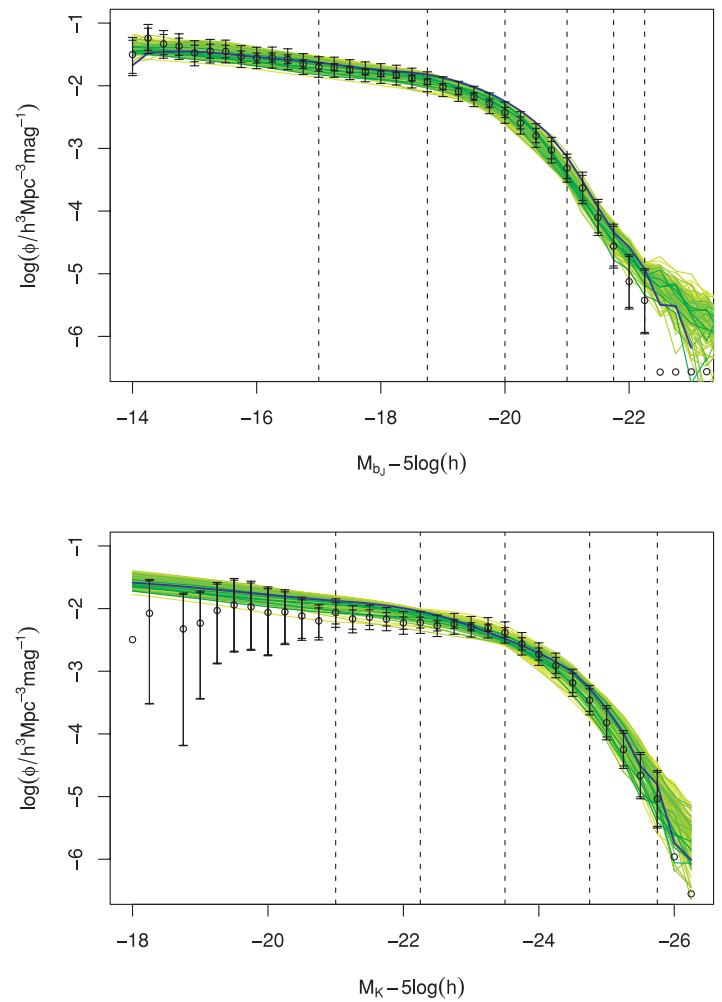

Figure 8. The $b_{\mathrm{J}}$ and $K$ luminosity functions of the 113 acceptable model evaluations found in Wave 5 . The axes, colour scale, error bars and lines are the same as in Fig. 5.

Table 6. Additional data sets used to constrain the model fits. Column 1: the property considered; Column 2: the data bins used in the comparison. The $\chi^{2}$ of the Bow06 model is given in Column 3. Column 4 gives the $\chi^{2}$ value for the best-fitting model for each separate comparison. In order to select an interesting set of models for further comparison between data sets, we used the $\chi^{2}$ cut-off value listed in Column 5 . Note that the cut-off values used for the disc size and gas mass to $L_{B}$ constraints are far in excess of those expected for a statistically acceptable model.

\begin{tabular}{lcccc}
\hline Data set & $N_{\text {bin }}$ & $\chi_{\text {Bow06 }}^{2}$ & $\chi_{\text {min }}^{2}$ & $\chi_{\text {cut }}^{2}$ \\
\hline Disc size & 40 & 7160 & 443 & 10000 \\
TF relation & 20 & 124 & 44 & 150 \\
Gas metallicity & 4 & 21 & 1 & 27 \\
Gas mass to $L_{B}$ & 9 & 3090 & 57 & 3500 \\
SDSS colours & 2400 & 16600 & 5150 & 17000 \\
BH mass & 8 & 5 & 3 & 60 \\
\hline
\end{tabular}

expect the model to perform in each test. For this reason, we focus on the performance of each model relative to Bow06. It should be noted, however, that Bow06 provides a poor match to the disc size data, the gas mass to luminosity data and SDSS colour data. In the case of the disc size data (cf. González et al. 2009), although the best-fitting models significantly improve the match to average sizes, they still fail to describe the variation with luminosity well, suggesting that the treatment of angular momentum in the code may need improvement. Bow06 also fails to reproduce the normalization of the gas mass to luminosity relation and the colours. However, the better fitting models result in a substantial improvement to these comparisons. The colours are significantly improved by increasing the yield $p_{\text {yield }}$, although the required value is larger than the best estimates based on stellar evolution models (cf. Cole et al. 2000) but within the range of their plausible uncertainty (cf. Font et al. 2008; Benson \& Bower 2010). We see below that the gas mass to luminosity ratio is sensitive to the assumed star formation efficiency (as was earlier found by Cole et al. 2000).

In a future application of the emulator method, we will apply the full emulator technique to encapsulate and hence emulate all of these statistical outputs. This needs to be combined with a careful analysis of the underlying statistical assumptions and the realistic model discrepancy terms. For the moment we aim only to make an indicative comparison, and we will only distinguish models which perform comparably well to Bow06 (the cut-off $\chi^{2}$ values are given in the table). It is important to note that $\chi^{2}$ cut-off values are not intended to denote a statistically acceptable fit to the data. As we will see, combining all these data sets in this way already restricts the parameter space substantially.

The dependence of $\chi^{2}$ for one of the data sets (disc sizes) is illustrated in Fig 10. Each panel shows the $\chi^{2}$ value as a function of one of the 16 input parameters. The Bow06 model is highlighted in red, while green points highlight models which fall below the cut-off $\chi^{2}$ value in all of the data sets tested. While a significant trend of $\chi^{2}$ (disc size) with $\epsilon_{\star}^{-1}$ is apparent, it is hard to discern a pattern in most of the panels. The lack of dependence may arise because the model output is not strongly dependent on a parameter, or because the dependence is masked by dependence on the other parameters. We describe our approach to systematic identification of interesting parameter combinations in Section 5.

We have plotted the parameter dependence of $\chi^{2}$ for each of the other data sets with similar results, and so do not reproduce each of the figures here. However, many properties exhibit a strong dependence on the parameter $\epsilon_{\star}^{-1}$, and we illustrate this in Fig. 11. The contradictory pressures on $\epsilon_{\star}^{-1}$ are apparent. Looking at the open and filled points together, it is clear that a better match to disc sizes is obtained by increasing this parameter; however, higher values of $\epsilon_{\star}^{-1}$ tend to worsen the match to the TF relation and the gas mass to luminosity ratios of the galaxies. The models highlighted in green are models which pass the $\chi^{2}$ cut-off in all of the data sets as well as provide an acceptable match to the luminosity functions. Thus, it is nevertheless possible to balance these opposing pressures, picking lower values of $\epsilon_{\star}^{-1}$ and exploiting other parameter dependencies to obtain adequate fits to the disc size. We discuss the implication of these results for future directions of the model in Section 6.2. It is notable that the green points all produce better matches to the SDSS colour data than Bow06 even though we did not enforce this in their selection (of course, we have enforced some colour information by requiring that models match both the $b_{\mathrm{J}}$ and $K$-band luminosity functions).

A final point to note from Fig. 10 is the hint of bimodality in the distribution of green points in the $\alpha_{\text {reheat }}$ and $V_{\text {hot,disc }}$ parameters. The two families of acceptable models correspond to models with very strong supernova feedback, but a cycling time for the galactic fountain close to the halo dynamical time, and models with weaker feedback but with cycling times substantially longer than the halo dynamical time. Bow06 belongs to the first family of models, while the second family is more typical of the earlier Durham models (Cole et al. 2000; Baugh et al. 2005) and models from the Munich group (e.g. Croton et al. 2006; De Lucia \& Blaizot 2007). Further model runs, and fuller treatment of the additional data sets, are required to explore this point further. 

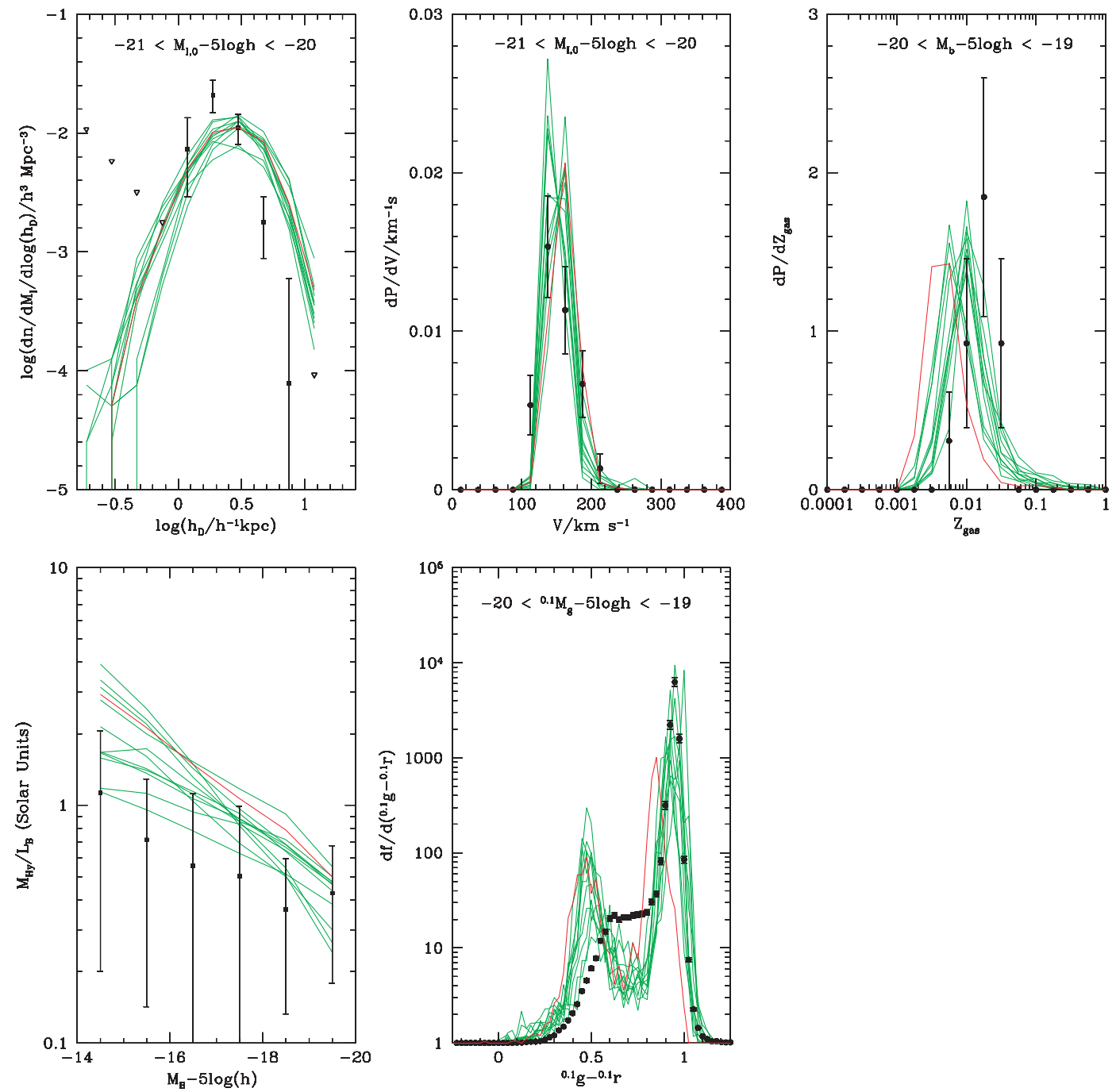

Figure 9. This figure illustrates the comparison of models with additional data sets. Models which give an acceptable match to the luminosity function data, and produce fits with $\chi^{2}<\chi_{\text {cut }}^{2}$ (see Table 6 ) for all of the additional data sets considered, are shown as green lines. The same models are highlighted as green points in Fig. 10. The Bow06 model is shown as a red line for comparison, and the observational data are shown as black points with error bars or upper limits (denoted by triangles). The source of the observational data is described in the text. With the exception of the gas mass to luminosity comparison, the panels show the comparison for a single magnitude slice. The total $\chi^{2}$ values shown are derived by summing the contributions from several magnitude slices.

\section{PROJECTION PURSUIT}

\subsection{Luminosity function constraints}

With such a high-dimensional data set, plotting the dependence of model runs as a function of one or two input parameters conveys only a small fraction of the complexity of the underlying parameter space. This bi-variate approach assigns a special significance to the input parameters and, thus, although it is not easily possible to present higher dimensional projections, we can make a more informed choice of the projection vectors. In particular, we can optimize the projection to show (1) the reduced dimensionality of the parameter space enforced by the constraints we have applied, and (2) maximize the additional leverage of the other data sets that we have considered in the previous section. This optimization is often referred to as 'projection pursuit'.

One approach is to project the parameter space of 'acceptable' models along its principal components. This effectively corresponds to rotating the region of acceptable models to align with the direc- tions of greatest to least variation of parameters. In many situations, PCA is used to find the directions with greatest variance; in our case, we are more interested in the directions with least variance. These are the parameter combinations that are most tightly constrained by the observational data. Using PCA we can align the hyperplanes revealed by our parameter space exploration so that their cross-section is viewed. It should be stressed, however, that PCA is inherently linear, and that the projected cross-section may hide a much thinner, but warped, relationship between the variables.

One potential problem of PCA is that the input variables must be scaled so that the variance along different axes can be compared. This is somewhat arbitrary. Our approach is to scale the variables by the initial search range, rather than restricting it to the range over which fits were found to be possible. By restricting our range in this way, we are aiming to determine whether the high dimensionality of the acceptable space can be reduced by suitable combination of parameters.

A principal component with low variance implies that this particular combination of the parameters is tightly constrained if the 


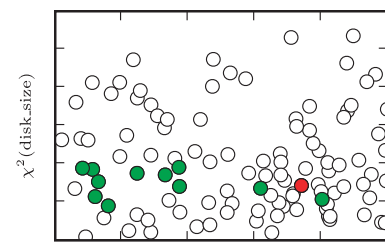

$V_{\text {hot,disk }}$
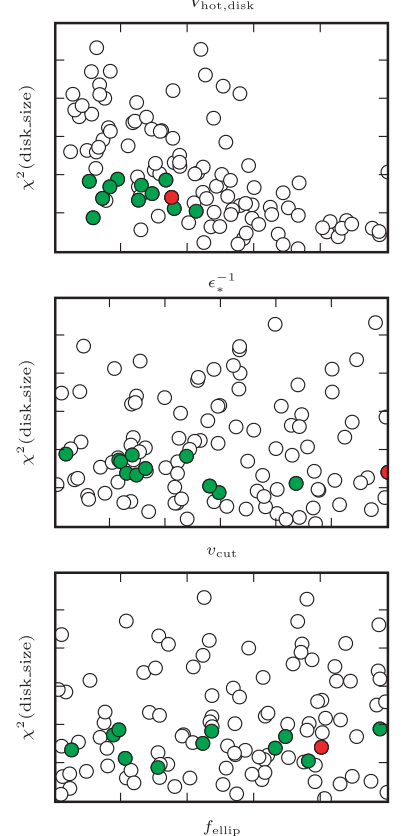

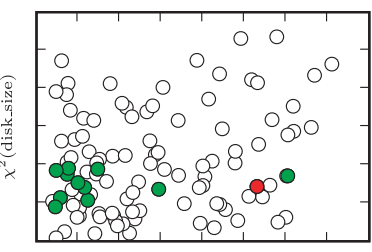

$\alpha_{\text {reheat }}$

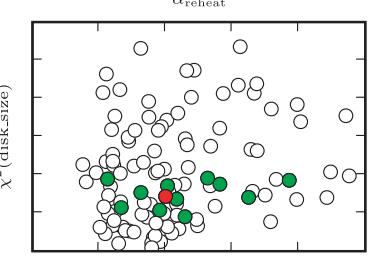

$f_{\text {stab }}$
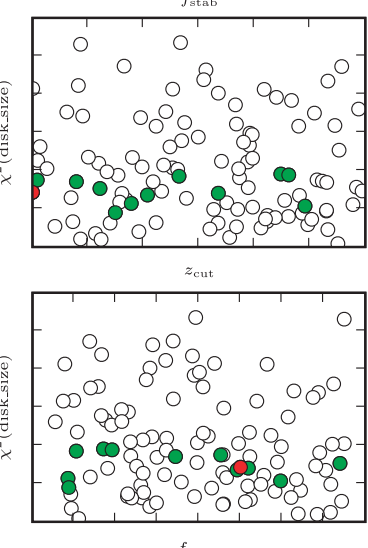

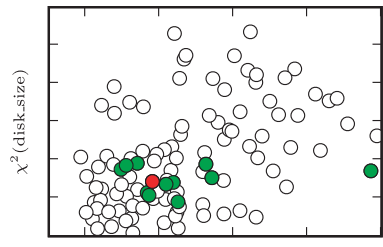

$\alpha_{\mathrm{cool}}$
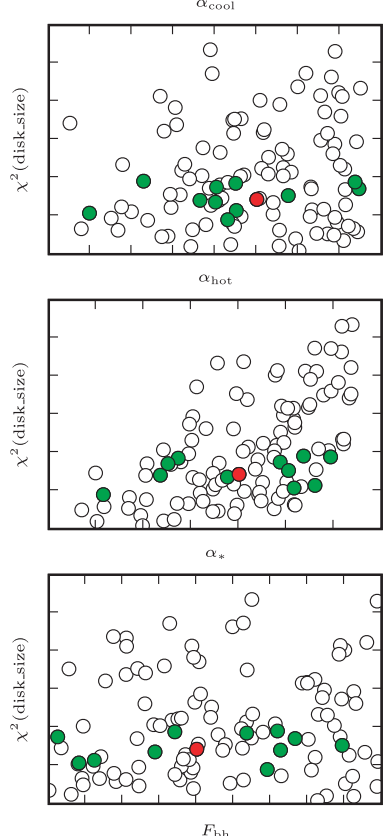

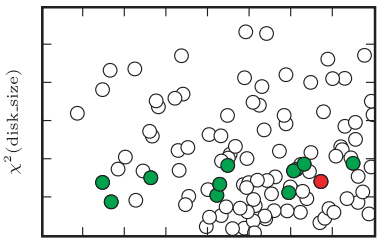

$V_{\text {hot, burst }}$
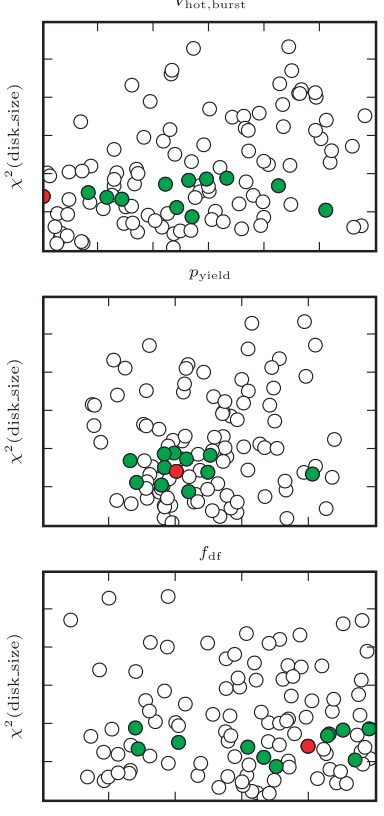

Figure 10. This plot shows the $\chi^{2}$ values of the models compared to the observed sizes of galaxy discs as a function of some of the more important parameters. The $y$-axis ranges from 0 to 30000 in each case, while the model parameters cover the range given in Table 1 . The Bow06 model is shown as a red filled circle. All the open points shown provide an acceptable match to the $b_{\mathrm{J}}$ and $K$ luminosity functions. Green points highlight models which have $\chi^{2}$ measures similar to or better than Bow06 when compared to all of the observational constraints listed in Table 6 . All the points shown (filled and open) produce acceptable fits to the $b_{\mathrm{J}}$ and $K$-band luminosity functions.

model is likely to produce an acceptable luminosity function fit. Of course, even if this constraint is satisfied, a good model is not guaranteed; rather we can be confident that if it is not satisfied the fit is unlikely to be good. When analysing the acceptable region in this way, we also need to bear in mind that the PCA assumes that the relationships are linear, whereas we have seen that the actual acceptable space is curved. This will prevent any of the suggested projections being arbitrarily thin and limit the accuracy of constraints.

We begin by focusing on the luminosity function data alone. Fig. 12 shows the result of applying the PCA to the 113 model runs which resulted in acceptable matches to the luminosity function. As with previous plots derived from luminosity function data alone, we consider only the 10 'active' variables identified by the emulator analysis. The relationship between the PCA variables and the original model parameters is given in Table 7.

We have previously seen that, by trading between parameters, acceptable solutions can be found over almost the full range of the input parameters. The PCA reveals a different story, however. In the plot, the grey region illustrates the original parameter space projected on to each pair of PCA components. The region is not square because the new coordinate system is not aligned with the axes of the original hypercube. The blue regions show the projection of models for which the luminosity function was acceptable. In this space, it is seen that many parameters are strongly constrained compared to their initial range. In the projections of the three most constrained parameters, the good fits shrink towards a point. For comparison, we show the Bow06 model as a red point. In many projections, it is evident that it lies well to the side of the main parameter space.

We can quantify the degree to which parameters have been constrained by comparing the range of the PCA components in the initial parameter space to the range covered by the acceptable models. Note that although all the input variable have been scaled to \pm 1 , the range of the PCA components may be greater because the component may be aligned with a diagonal of the hypercube. The comparison of the ranges is shown in Fig. 13. The figure quantifies the degree to which the components are constrained. We consider the reduction in range, since the ratio of the standard deviation is sensitive to the orientation of the original hypercube. However, similar results are obtained when we consider the ratio of standard deviation of the PCA components. Three components stand out in particular, with ranges that are less than $1 / 4$ of their initial range. In order to have an adequate match to the luminosity function these components must have quite precisely determined values. However, simply matching these values does not guarantee a fit to the data, since the other components also need to be considered. Indeed, all of the PCA components cover a reduced range compared to the initial parameter space.

It is important to emphasize again that PCA cannot extract the full interdependence of the parameters since the analysis is intrinsically linear. Fig. 14 illustrates the limitations. The crescent shape arises because the observational constraints generate a non-linear 

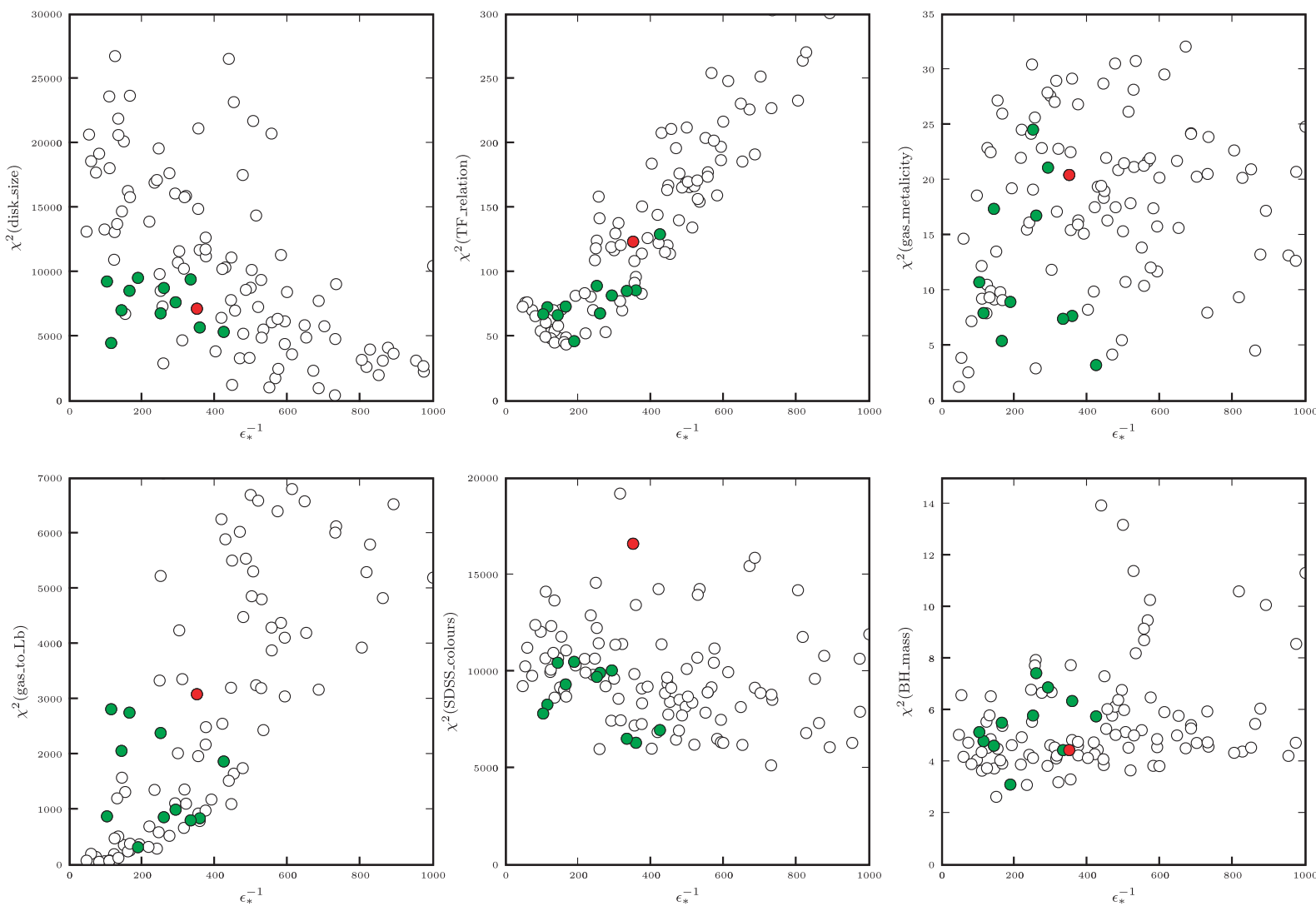

Figure 11. The variation in $\chi^{2}$ for various data constraints as a function of the $\epsilon_{\star}^{-1}$ parameter. From top left to bottom right, the $y$-axes show the $\chi^{2}$ derived from comparison of disc sizes, the TF relation, gas metallicity, gas mass to luminosity ratio, SDSS colours and black hole mass. The colours of data points are the same as in Fig. 10.

dependence between the components of Var 2 and Var 10. The PCA has selected these directions to minimize the linear variance. On the other hand, although the emulator analysis includes terms up to third order, it is unclear how the physical dependencies can be extracted. Thus, despite its limitations, PCA provides a good means of gaining insight into the physical processes driving the match to the luminosity function.

The relationship between the PCA variables and the original model parameters is given in Table 7. This expresses each PCA component as a vector direction in the scaled input variables. The components have all been normalized to unit length in the 10D space so that a large coefficient indicates that the PCA component direction is closely aligned with the input variable. Without first applying the scaling given in Table 1 the relative importance would be obscured. We consider the weight of the input variables in the most constrained components below: although none of the components is completely aligned with the input parameters, most have a dominant component that can be identified with one particular input variable. These are highlighted in the table. Below we consider the three most constrained PCs, focusing on components with contribution greater than 0.3 .

Var 10 is dominated by a 70 per cent contribution from $V_{\text {hot,disc }}$. This parameter controls the amount of mass ejected from the galaxy disc to the halo for each solar mass of stars formed. However, an adequate match to the luminosity function may be maintained if an increase in $V_{\text {hot,disc }}$ is offset by an increase in $\alpha_{\text {reheat }}$ (decreasing the time-scale for the material to become available for reaccretion), decreasing $\alpha_{\text {cool }}$ (increasing the mass scale at which AGN feedback becomes important) and/or decreasing $\alpha_{\text {hot }}$ (reducing velocity scaling of the mass ejected from the disc).

Var 9 is dominated by a 70 per cent contribution from $\alpha_{\text {cool }}$. Increases in $\alpha_{\text {cool }}$ can be offset by reducing the star formation efficiency (through $\epsilon_{\star}$ ) or increasing the mass dependence of the disc feedback (through $\alpha_{\text {hot }}$ ). This PCA component also has important contributions from $V_{\text {hot,disc }}$ (with the opposite sign to the cross term in Var 10) and the disc stability criterion. The physical significance of the Var 9 becomes clearer if we consider the effect of adding a small contribution from Var 10 to create a new component that is independent of $V_{\text {hot,disc }}$. This strengthens the dependence on $\alpha_{\text {cool }}$, while slightly weakening the dependence on $\alpha_{\text {hot }}$. We explore the idea of optimally rotating principal components in Section 6.2.

Var 8 is dominated by the disc stability criterion, $\epsilon_{\mathrm{disc}}$, with significant contributions from the star formation rate efficiency, $\epsilon_{\star}$, and the mass dependence of feedback efficiency, $\alpha_{\text {hot }}$.

Looking at the less constrained components, we see that these often have dominant input variables too. Var 6 and Var 4 are dominated by $f_{\text {df }}$ (the dynamical friction time-scale), Var 5 by the yield (increasing the metal abundance normalization), Var 3 by $\alpha_{\star}$ (the star formation law exponent), Var 2 by $\alpha_{\text {reheat }}$ (the time-scale for re-incorporating ejected disc gas). Var 1, the least constrained component, has equally strong contributions from the yield and the star formation rate efficiency.

We have ordered the variables in Table 7 so that the most constrained dominant parameters appear first. This emphasizes that the most important parameters are $V_{\text {hot,disc }}, \alpha_{\text {cool }}$ and $f_{\text {stab. }}$ It is also 


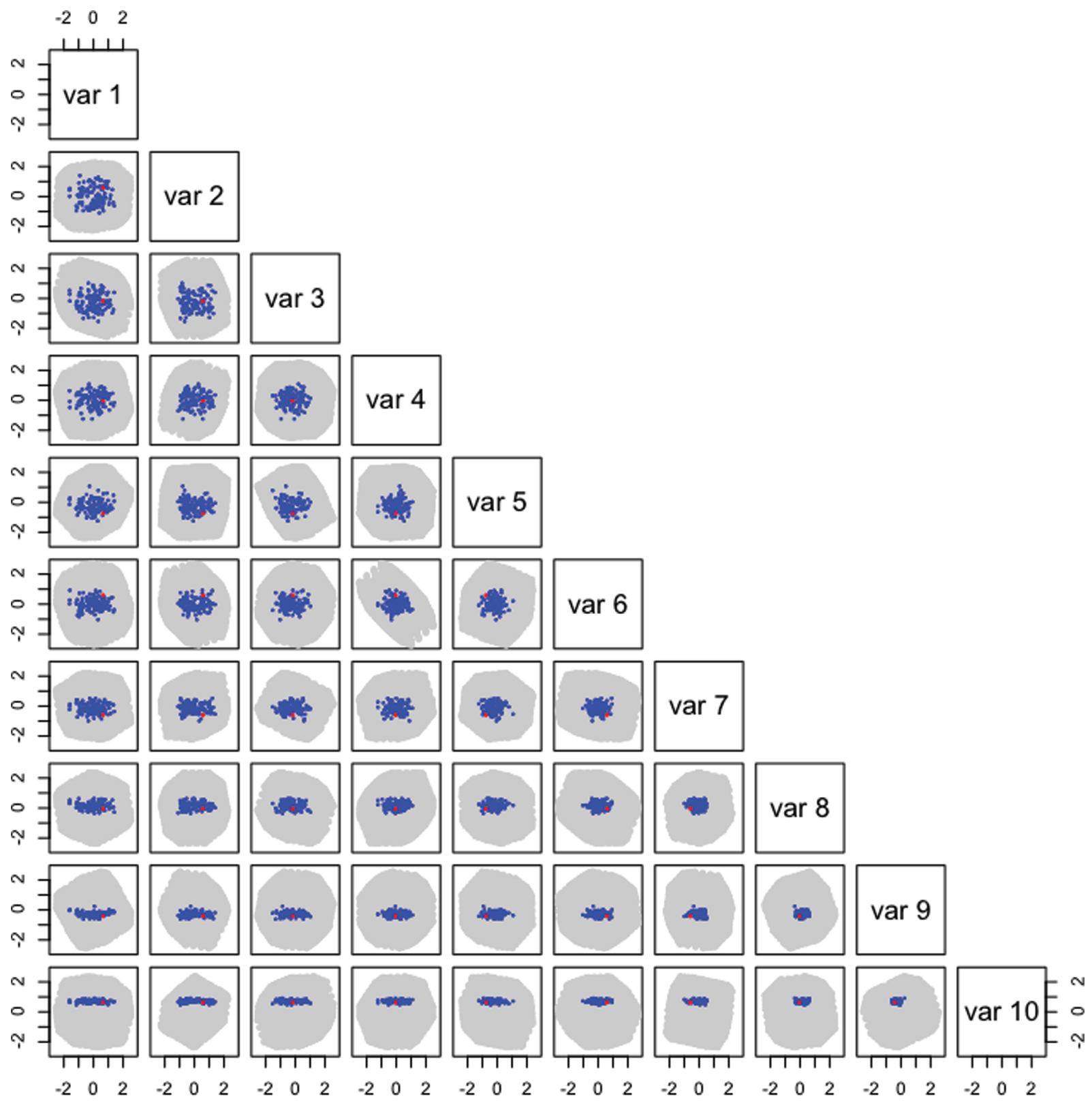

Figure 12. The space of acceptable models that reproduce the local $b_{\mathrm{J}}$ and $K$ luminosity functions projected along pairs of principal components. Blue points show models which produce luminosity functions with implausibility less than 2.5 . Grey points show the geometry of the initial allowed parameter space in each of these projections. The principal components are chosen to minimize the variance of the models with low values of $\chi^{2}$. The highest components (e.g. Var 10) are the most constrained. The Bow06 model is shown as a red filled circle. This figure illustrates the reduction in the dimensionality of parameter space resulting from requiring a good match to a particular LF.

notable that $\alpha_{\text {hot }}$ (the mass dependence of the feedback) is not dominant in any particular component, but makes an important contribution to many of them.

\subsection{Important directions from other data}

Having shown that projection pursuit using PCA provides a useful way of capturing the geometry of the region producing acceptable luminosity functions, we proceed to apply a second level of analysis to examine how the introduction of additional constraints further restricts the allowed region of parameter space.

In order to perform this analysis, we first express each model as a function of the principal components identified by the luminosity function constraints, as described in Section 5.1. We then renormal- ize these components so that their variance is equal (and recentre the distribution on the mean). This has the effect of mapping the distribution of acceptable parameter region (according to the luminosity function criterion) into a roughly spherical distribution centred on the origin. We then further restrict the data set using the additional data, keeping only those runs with low $\chi^{2}$, and characterize this reduced space in terms of its new principal components. We then map these vectors back into the space defined by the scaled input parameters, providing insight into the physical differences between models that satisfy both the luminosity function constraints and the additional data and those that do not. The coefficients quoted in Table 8 are the coefficients of the scaled input variables in the additionally constrained direction. The scalings of the original input parameters are given in Table 1 . 
Table 7. Principal components for the acceptable space of luminosity functions. The columns give the PCA variables ordered by decreasing relative standard deviation, where the relative standard deviation is the standard deviation of the component when the initial range of variables has been scaled to the range \pm 1 . Small relative standard deviations correspond to components that are tightly constrained by the requirement of producing a good luminosity function. Dominant input variables in each of the vectors are highlighted in bold font. The variables have been ordered so that the most constrained components appear last.

\begin{tabular}{ccccccccccc}
\hline & Var 1 & Var 2 & Var 3 & Var 4 & Var 5 & Var 6 & Var 7 & Var 8 & Var 9 & Var 10 \\
\hline$\tilde{V}_{\text {hot, disc }}$ & 0.198 & 0.112 & -0.231 & -0.283 & 0.334 & 0.311 & 0.0637 & -0.114 & -0.256 & $\mathbf{0 . 7 2 4}$ \\
$\tilde{\alpha}_{\text {cool }}$ & -0.386 & 0.361 & 0.136 & -0.00109 & -0.00943 & -0.0733 & 0.0977 & -0.265 & $\mathbf{0 . 7 1 3}$ & 0.33 \\
$\tilde{f}_{\text {stab }}$ & -0.149 & -0.147 & 0.247 & -0.312 & -0.0218 & 0.331 & -0.104 & $\mathbf{0 . 7 8 1}$ & 0.236 & 0.103 \\
$\tilde{V}_{\text {hot,burst }}$ & -0.115 & -0.167 & 0.414 & -0.234 & 0.102 & 0.173 & $\mathbf{0 . 7 8 3}$ & -0.181 & -0.135 & -0.168 \\
$\tilde{\tau}_{0, \text { mrg }}$ & -0.203 & 0.0895 & 0.0975 & $\mathbf{0 . 7 2 9}$ & 0.0545 & $\mathbf{0 . 6 2 7}$ & -0.0033 & 0.00626 & -0.116 & 0.0238 \\
$\tilde{p}_{\text {yield }}$ & $\mathbf{- 0 . 5 8 1}$ & 0.043 & -0.263 & 0.0517 & $\mathbf{0 . 6 3 2}$ & -0.3 & 0.0585 & 0.223 & -0.183 & -0.109 \\
$\tilde{\alpha}_{\star}$ & 0.214 & 0.0194 & $\mathbf{0 . 7 1 8}$ & 0.0465 & 0.49 & -0.152 & -0.392 & -0.127 & -0.0453 & 0.0239 \\
$\tilde{\alpha}_{\text {reheat }}$ & 0.0702 & $\mathbf{0 . 7 8 0}$ & -0.031 & -0.313 & 0.051 & 0.273 & -0.0775 & 0.0104 & -0.104 & -0.44 \\
$\tilde{\epsilon}_{\star}^{-1}$ & $\mathbf{0 . 5 8 9}$ & 0.111 & -0.166 & 0.297 & 0.348 & -0.102 & 0.383 & 0.302 & 0.387 & -0.082 \\
$\tilde{\alpha}_{\text {hot }}$ & -0.013 & 0.421 & 0.27 & 0.213 & -0.333 & -0.41 & 0.229 & 0.341 & -0.379 & 0.337 \\
Range ratio & 0.598 & 0.554 & 0.496 & 0.469 & 0.487 & 0.357 & 0.343 & 0.21 & 0.167 & 0.0945 \\
Mean & 0.00341 & 0.00371 & -0.265 & 0.0368 & -0.244 & 0.00854 & -0.132 & 0.153 & -0.279 & 0.697 \\
Rel. Std. Dev. & 0.679 & 0.65 & 0.564 & 0.487 & 0.418 & 0.393 & 0.323 & 0.224 & 0.145 & 0.0854 \\
\hline
\end{tabular}

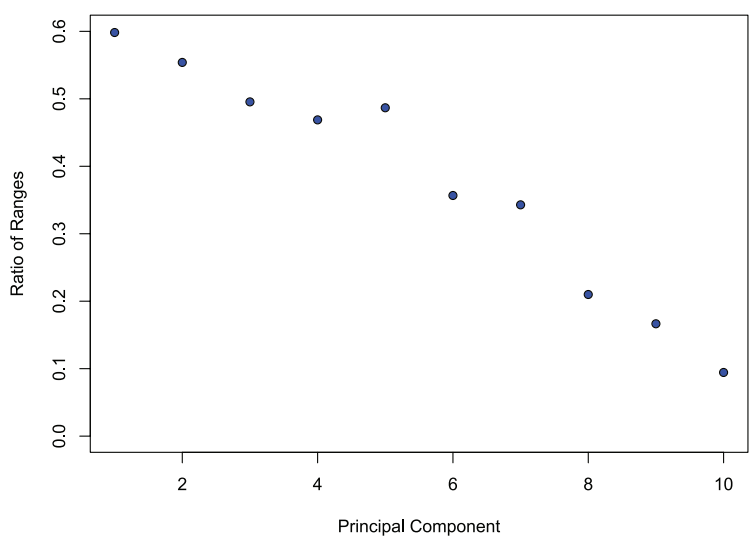

Figure 13. The reduction in the range of the PCA components from requiring that the model produces a low implausibility luminosity function. The PCA components are ordered by their variance, while the vertical axis shows the ratio of the range of acceptable models to the range of the PCA components in the initial parameter space.

We find that the analysis reveals significant constraints that arise from adding data on the disc sizes, the $M_{H I}$ to $L_{B}$ ratio and the TF relation. The dependence of $\chi^{2}$ on the most constrained component is shown for each of the data sets in Fig. 15. The importance of these data sets was already apparent because of the clear dependence on $\epsilon_{\star}^{-1}$ in Fig. 11; however, the PCA is capable of revealing constraints that are based on combinations of parameters that would not have been evident in a simpler analysis. For example, contrast the evident bunching of the low $\chi^{2}$ points in the first panel [which shows $\chi^{2}$ (disc size)] with the absence of clear trends in Fig. 10.

The reduction in the standard deviation due to the PCA is shown in Table 8, together with the model's additionally constrained directions. It is immediately apparent that the directions due to the gas mass to $L_{B}$ ratio and the TF relation constraints are similar. Not only do both have dominant contributions from $\epsilon_{\star}^{-1}$, but the weighting of most other variables are also similar.

While the disc size constraint also depends strongly on $\epsilon_{\star}^{-1}$, the overall direction of the constraint is different from that implied by the Gas to $L_{B}$ ratio, and the TF relation, and the constrained direc-

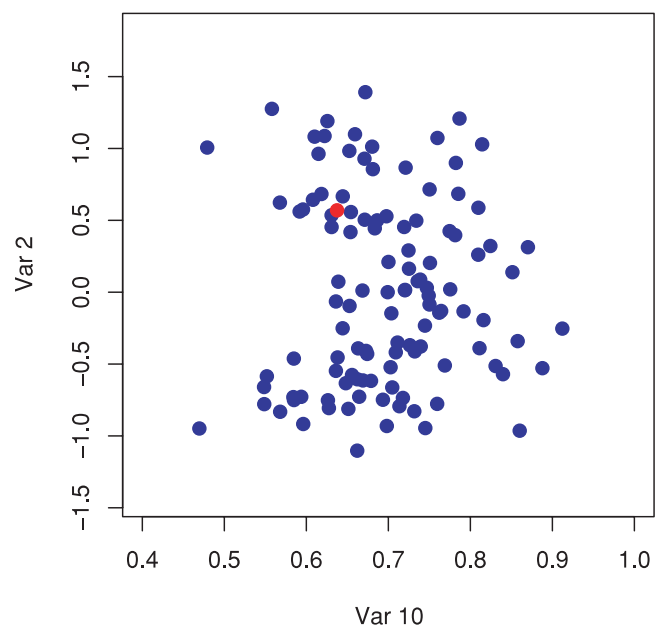

Figure 14. Close up of one PCA projection, showing that the acceptable space is not linear, and cannot be accurately described by the principal components.

tion also depends equally strongly on the yield and $\alpha_{\text {cool }}$ parameters. Thus, models which match all the data sets tend to have low values of $\epsilon_{\star}^{-1}$, selecting particular values for the other parameters to compensate for this. This is evident in Fig. 11, and explains the trend for the accepted models to be squeezed into the lower left corner of the first panel. This tension has also been apparent in earlier versions of the GALFORM code (e.g. Cole et al. 2000); however, the analysis scheme presented here provides an objective means of identifying the interplay between observational constraints and model parameters.

\section{DISCUSSION}

In this paper, we have set out to explore the parameter space of the GALFORM semi-analytic model. The model implements the key physical processes that define the formation of galaxies, including the hierarchical growth of dark matter haloes, gas accretion and cooling, and the feedback effects of supernovae and AGN. The model we use contains 16 parameters that describe these processes. 
Table 8. The most additionally constrained directions using various complimentary data sets in addition to the luminosity function constraints. For each data set, the table shows the most constrained component vector together with the factor by which the standard deviation is reduced. Directions are quoted relative to the normalized parameter range: the scaling is given in Table 1. These components provide physical insight into the effect of introducing the additional data sets. Only data sets which resulted in a significant additional constraint are shown: the other data sets considered in Section 4.3 do not result in strong additional linear constraints on parameter combinations. Parameters with no contribution greater than 0.2 have been suppressed. Note that the best constrained directions are similar for the TF and gas mass to luminosity data sets.

\begin{tabular}{ccccccccc}
\hline & Relative Std. Dev. & $\tilde{V}_{\text {hot,disc }}$ & $\tilde{\alpha}_{\text {cool }}$ & $\tilde{\epsilon}_{\star}^{-1}$ & $\tilde{f}_{\text {stab }}$ & $\tilde{p}_{\text {yield }}$ & $\tilde{\alpha}_{\star}$ & $\tilde{\epsilon}_{\text {Edd }}$ \\
\hline Disc size & 0.49 & 0.301 & -0.519 & 0.468 & -0.119 & -0.449 & -0.269 & -0.021 \\
Gas mass to $L_{B}$ & 0.40 & 0.16 & -0.251 & $\mathbf{0 . 7 6 4}$ & -0.282 & -0.197 & -0.334 & -0.205 \\
TF relation & 0.29 & 0.264 & -0.33 & $\mathbf{0 . 7 7 5}$ & -0.207 & -0.202 & -0.24 & -0.196 \\
\hline
\end{tabular}
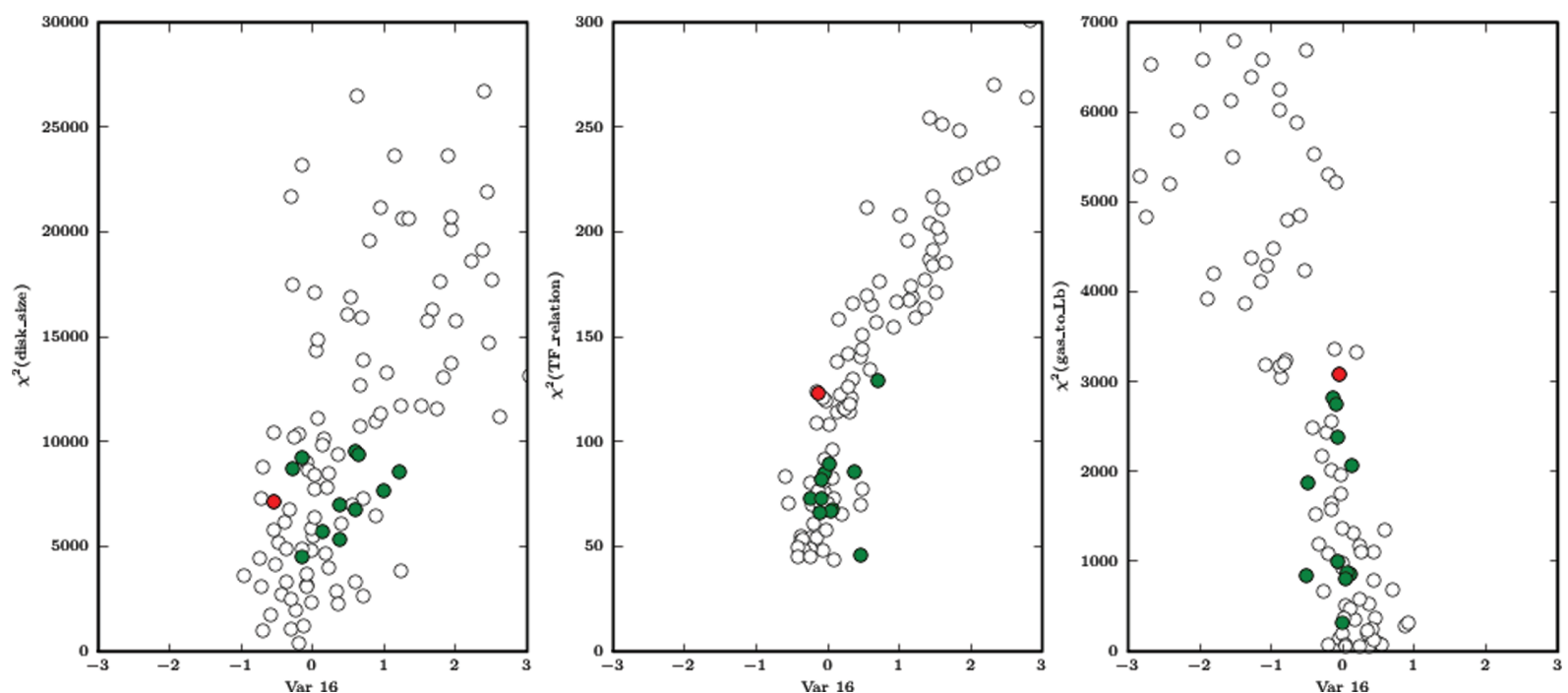

Figure 15. We show the $\chi^{2}$ values of the models as functions of some of the most additionally constrained principal components identified in Table 8. In each panel, we show the most constrained component ( $x$-axis) resulting from requiring the model to match the data sets shown on the $y$-axis. Note that the vector direction represented by the axis is different in each plot (see Table 8). The Bow06 model is shown in red. Green solid points highlight models which gave fits comparable to, or better than, Bow06 in all the tests given in Table 6. Note how the chosen projection results in a bunching of the low $\chi^{2}$ models compared to the full set. All the points plotted produce an acceptable match to the luminosity function data.

Some parameters have strongly constrained, physically plausible values; others represent poorly understood physical processes and are only weekly constrained by the observational data used here. Bow06 identified a largely successful point in this parameter space by matching the local $b_{\mathrm{J}}$ - and $K$-band luminosity functions. In this paper we set out to find out how unique this point is and to explore whether other parameter combinations could perform equally well (or better) at reproducing local galaxy properties.

\subsection{The model emulator technique}

We have adopted the Model Emulator technique to efficiently but conservatively search the $16 \mathrm{D}$ parameter space. The essence of the approach is to build a statistical predictor for model results on the basis of a limited set of model runs. This is a Bayesian approach, where we are aiming to quantify the information we derive from the runs. We use the statistical model to identify regions where we are confident that an acceptable fit will not be found. Such regions are denoted as 'implausible' and excluded from further consideration. A second wave of emulation is then performed to better characterize the surviving portion of parameter space. After four waves of emulation, we are left with an accurate emulation of the model that defines a 'not implausible' region containing 0.26 per cent of the original volume. A run within this region is not guaranteed to give a statistical match to the observational data, and subsequent runs within this region are needed to identify acceptable model realizations.

In this paper, we have focused on matching to the observed $b_{\mathrm{J}}$ - and $K$-band luminosity functions of galaxies in order to parallel the approach used in Bow06. An important issue has been to quantify the observational and model uncertainties. A key concept has been that of model discrepancy. This term accounts for the expected accuracy of the model itself: since the semi-analytic model is inherently an approximation to the true physical process, we are willing to accept models which come close to the observational data but do not match it exactly. This is a key concept in our Bayesian approach, and it differs from most previous work in which the philosophy is to adapt the model parameters to find fits consistent within the observational uncertainties alone. We argue that it is important to explicitly account for the approximate nature of the model, and that ignoring the model discrepancy term will lead to overzealous exclusion of some regions of parameter space. In this sense, the model discrepancy term makes our approach conservative. Naturally, it is potentially of interest to investigate the effect of reducing the model discrepancy term in order to guide improvements to the model. However, we feel that improvements in the model are currently better driven by comparison to additional data sets, where the tensions in the 
model can be more easily exposed (as illustrated in Section 4.3). Formal discussion of the process of 'model reification' can be found in Goldstein \& Rougier (2009).

We have accepted models at the $2.5 \sigma$ level, which equates to models that come within a factor of approximately 1.54 of the observed luminosity function (i.e. a factor of $10^{2.5 \times 0.0753}$; see Section 3.6). Since the dynamic range of the luminosity function covers almost 5 orders of magnitude in space density this term appears relatively small in any visual comparison, as can be seen from Fig. 8. Acceptable luminosity functions are generally very good matches to the luminosity function around the knee of the luminosity function. It is evident that many models struggle to match the very bright part of the $b_{\mathrm{J}}$ luminosity function, even though they provide a good description of the break in the $K$-band luminosity function. Physically, these models tend to allow a small amount of star formation to take place in the most massive systems.

Nevertheless, the model emulator suggests that plausible matches to the luminosity functions may be found over a large fraction of the parameter ranges. However, this results from a fine-tuned interplay between parameters and the acceptable models are confined to thin hyperplanes. Out of the 16 input variables, we find that 10 need to be taken into account in order to make the emulator predictions. These are labelled 'A' in Table 1 . The remaining parameters $\left(\epsilon_{\text {Edd }}, f_{\text {ellip }}, f_{\text {burst }}, F_{\text {bh }}, v_{\text {cut }}\right.$ and $\left.z_{\text {cut }}\right)$ have a weak effect on the luminosity functions (although it may have significant impact on other aspects of the model). Although we have achieved a large reduction in parameter space, further model runs need to be performed to determine whether each point actually returns a statistically acceptable luminosity function. Out of a sample of 2000 runs surviving the Wave 4 implausibility cut-off, we find 113 runs that provide acceptable fits to the luminosity function, implying that only 0.014 per cent of the initial input parameter space is compatible with the combined observational constraints and model discrepancy terms.

Although the model emulator technique is gaining favour in other areas of scientific modelling, we could have applied the MCMC method to the problem. This approach has been pursued by Henriques et al. (2009) and Kampakoglou et al. (2008). However, they consider only a small subset of the possible parameters, analysing a 6D and 7D space, respectively, while we consider 16D space. We note that while 'only' 10 active variables are included in our fitting functions, the full parameter space is considered by the emulator. The active variables do not need to be guessed in advance of the minimization process, and are all varied in all our GALFORM runs. Moreover, the approach we adopt is readily adapted as introducing new physical processes in the GALFORM model generates even higher dimensionality (e.g. Baugh et al. 2005; Benson \& Bower 2010).

Our galaxy formation model is most comparable to that used by Henriques et al. (2009) (which is based on Croton et al. 2006). Comparison of the results is, however, complicated because several of their chosen parameters do not have direct equivalents in our code and their analysis does not allow for any model discrepancy term. Nevertheless, their results for luminosity function constraints are qualitatively similar to ours, given the lower dimensionality of their investigation. A significant difference is that they find that including constraints on the black hole mass-bulge mass correlation strongly constrains the parametrization. In contrast, we find that this relation adds little constraint. This arises from the very different treatment of black hole feedback in the two models: with the larger number of parameters that we consider, we find that the black hole mass may be adjusted largely independently of the galaxy luminosity function. This illustrates the potential danger of including too few dimensions in the analysis, since this artificially restricts the dimensionality of the problem. As we have stressed, parameters should be allowed freedom to vary even if their prior distributions are significantly constrained. If the emulator finds that the prior range has little impact on the model output, it will reject the parameter from further consideration. This is not a qualitative decision that should be made at the outset. In future models, we will also allow the cosmological parameters to vary within their prior distributions. This is difficult if the model is driven by $N$-body simulations, but is possible if it is driven by the improved Monte Carlo merger-tree generators such Parkinson, Cole \& Helly (2008). In principle, the MCMC method (e.g. Trotta 2008) could be applied to higher dimensionality problems, however, it then becomes hard to drive convergence of the MCMC chains. In the statistical model fitting literature, these scaling problems have meant that MCMC is falling out of favour, being replaced by the emulator techniques that we have applied here, which were specifically designed to deal with high-dimensional models (e.g. Oakley \& O'Hagan 2004; Heitmann et al. 2009). As the dimensionality of the GALFORM model increases further (Benson $\&$ Bower 2010) the advantages of the emulator technique become even more relevant.

Our experience in $16 \mathrm{D}$ space suggests that the techniques could be extended to even higher dimensions, with a relatively modest increase in computing effort. To arrive at the emulation presented here, we performed 5500 model runs. This was by no means the minimum number of evaluations, and we have tended to be very conservative in the strategy adopted. A number of techniques could be used to speed up the calculations, for example performing smaller, low accuracy runs for the initial waves, increasing the accuracy as the not implausible region shrinks. An important aspect of the emulator technique is that variables are only explicitly included in the emulator, when the statistical improvement in doing so is justified. Thus, adding irrelevant (and even degenerate) variables does not unduly handicap the method.

\subsection{Projection pursuit}

The emulator technique provides a reliable statistical description of the GALFORM model. This allows rapid estimation of the likely success of different regions of parameter space. However, the statistical model does not easily provide insight into the physical interactions between parameters. PCA of the successful runs provides a complimentary analysis, allowing us to orient the parameter space so as to reveal the most tightly constrained model parameters. We find that three directions are strongly constrained, corresponding to linear combinations of the $V_{\text {hot,disc }}, \alpha_{\text {cool }}, \epsilon_{\star}^{-1}, \alpha_{\text {reheat }}$ and $\alpha_{\text {hot }}$. This makes good physical sense: increasing the strength of disc feedback needs to be balanced by reducing the time-scale for reheated gas to fall back to the disc, for example. Setting these parameters to an appropriate combination is necessary to arrive at good models but it is not sufficient, and we stress that all 10 active parameters play a significant role.

As well as providing physical insight, the PCA components provide a means of orienting future parameter space exploration. For example, if we were to start from a single acceptable model, we can attempt to generate another by moving along the PCA hyperplanes. If we select a value for $\tilde{V}_{\text {hot,disc }}$, Var 10 (in Table 9) suggests we adopt a particular combination of $\tilde{\alpha}_{\text {cool }}, \tilde{\alpha}_{\text {reheat }}$ and $\tilde{\alpha}_{\text {hot }}$ (plus smaller contributions from the other parameters). Since $V_{\text {hot,disc }}$ is fixed, Var 9 and Var 8 provide two additional linear equations linking (primarily) $\tilde{\alpha}_{\mathrm{cool}}, \tilde{\epsilon}_{\star}^{-1}$ and $\tilde{\alpha}_{\text {hot }}$. By choosing one of the four variables, we can invert the system of equations and arrive at a good 
Table 9. The three most constrained PCA components have been combined to diagonalize the constraints on $V_{\text {hot,disc }}, \alpha_{\text {cool }}$ and $f_{\text {stab }}$. We use the notation $\operatorname{Var}^{\prime \prime} 8$ (etc.) to emphasize that these variables are distinct from those in Table 7. This rotation makes the physical dependence of these strongly constrained variables evident (see text for details). Coefficients larger than 0.2 have been highlighted in bold. Note that these directions are not normalized.

\begin{tabular}{cccc}
\hline & $\operatorname{Var}^{\prime \prime} 8$ & $\operatorname{Var}^{\prime \prime} 9$ & $\operatorname{Var}^{\prime \prime} 10$ \\
\hline$\tilde{V}_{\text {hot,disc }}$ & & & 1 \\
$\tilde{\alpha}_{\text {cool }}$ & & 1 & \\
$\tilde{f}_{\text {stab }}$ & 1 & & \\
$\tilde{V}_{\text {hot, burst }}$ & $\mathbf{0 . 3 0}$ & -0.14 & 0.13 \\
$\tilde{\tau}_{0, \mathrm{mrg}}$ & 0.03 & -0.11 & -0.08 \\
$\tilde{p}_{\text {yield }}$ & 0.17 & $\mathbf{0 . 3 2}$ & -0.03 \\
$\tilde{\alpha}_{\star}$ & 0.16 & 0.0 & -0.05 \\
$\tilde{\alpha}_{\text {reheat }}$ & -0.14 & $\mathbf{0 . 2 6}$ & $-\mathbf{0 . 4 7}$ \\
$\tilde{\epsilon}_{\star}^{-1}$ & $\mathbf{0 . 4 4}$ & $\mathbf{- 0 . 2 9}$ & $\mathbf{0 . 3 0}$ \\
$\tilde{\alpha}_{\text {hot }}$ & $\mathbf{0 . 3 7}$ & $\mathbf{0 . 4 3}$ & $\mathbf{0 . 6 2}$ \\
Mean & 0.29 & -0.13 & 0.99 \\
\hline
\end{tabular}

Table 10. Equations describing the broad brush interactions between the most constrained parameters. These relations have been derived from Table 9. Note that these relations are highly approximate and only capture a small fraction of the behaviour described by the model emulator.

\begin{tabular}{lll}
\hline$V_{\text {hot,disc }}$ & $=$ & $550+110 \tilde{\alpha}_{\text {reheat }}+70 \tilde{\epsilon}_{\star}^{-1}-140 \tilde{\alpha}_{\text {hot }}\left(\mathrm{km} \mathrm{s}^{-1}\right)$ \\
$\alpha_{\text {cool }}$ & $=$ & $0.64+0.16 \tilde{p}_{\text {yield }}+0.13 \tilde{\alpha}_{\text {reheat }}-0.14 \tilde{\epsilon}_{\star}^{-1}+0.22 \tilde{\alpha}_{\text {hot }}$ \\
$f_{\text {stab }}$ & $=$ & $0.84-0.04 \tilde{V}_{\text {hot, burst }}-0.07 \tilde{\epsilon}_{\star}^{-1}-0.06 \tilde{\alpha}_{\text {hot }}$ \\
\hline
\end{tabular}

'guess' for an acceptable model. For example, we have already suggested that angular momentum transport in the model needs to be improved. This would result in denser gas discs and thus a change in the disc dynamical time. However, from outside the galaxy this change would be broadly like a shift in the effective value of the $\epsilon_{\star}^{-1}$ parameter.

We can investigate this further by noting that the variance of Var 8, 9 and 10 is similar. Together, they define the directions of a 7D hyperplane within which the model is significantly less well constrained, while it is strongly constrained in the three perpendicular directions. However, we can select a new linear combination of these components and define the seven-plane equally well. ${ }^{5}$ In particular, we can choose to 'diagonalize' the contributions from $\tilde{V}_{\text {hot,disc }}, \tilde{\alpha}_{\text {cool }}$ and $\tilde{f}_{\text {stab }}$, the variables with the largest contributions to the PCA components. The result of applying the diagonalization procedure is to define the new component vectors given in Table 9. If we now drop terms with coefficients less than 0.2 , we can arrive at a simple (but approximate) description of how $\tilde{V}_{\text {hot,disc }}, \tilde{\alpha}_{\text {cool }}$ and $\tilde{f}_{\text {stab }}$ should vary as a function of the other active variables in order to keep within the seven plane. These equations are given in Table 10, where we have translated the scaled variables back to their original units in order to make the physical dependence more apparent. Because we eliminated terms with small coefficients, $\tilde{\tau}_{0, \mathrm{mrg}}$ and $\tilde{\alpha}_{\star}$ do not appear in these expressions. Examining the equations gives useful insight into the interplay of the parameters. For example, it shows that the most successful models require very high values for the feedback parameter $V_{\text {hot,disc }}$, close to the maximum

\footnotetext{
${ }^{5}$ If the variances had been equal, the PCA components would be degenerate and all linear combinations would be equivalent. We could visualize a threedisc lying within the seven-plane. Since the variances are not exactly equal, the three-disc is distorted slightly into an ellipsoid.
}

value allowed in our analysis. In order to match the luminosity function with a lower value, low values must be chosen for $\tilde{\alpha}_{\text {reheat }}$ (the time-scale on which reheated gas fall back into the galaxy must be increased) and $\tilde{\epsilon}_{\star}^{-1}$ (the disc star formation rate must be reduced) and high values for $\tilde{\alpha}_{\text {hot }}$ (the relative strength of feedback in low-mass galaxies is increased). Similarly, we see that high values for $\tilde{\alpha}_{\text {hot }}$ imply that $\tilde{\alpha}_{\text {cool }}$ must be increased (increasing the mass at which the radio-mode feedback operates); however, decreases in both $\tilde{\alpha}_{\text {reheat }}$ and $\tilde{\epsilon}_{\star}^{-1}$ have a compensating effect on $\alpha_{\text {cool }}$. The appropriate choice of $\alpha_{\text {cool }}$ is also dependent on $\tilde{p}_{\text {yield }}$, as would be expected from the metallicity dependence of the cooling function.

These trends can be confirmed by looking at Fig. 4, however it is evident that the description of the trends in terms of a few components loses a great deal of the true complexity of the underlying parameter space. Thus, while consideration of the PCA components provides a useful guide to how we should remap the parameter space in order to capture the best-fitting parameter region, it must be remembered that PCA is fundamentally linear and its description is very approximate. The restriction to linear dependencies does not apply to the emulator technique.

\subsection{Additional data sets}

In this paper, we have followed the approach of Bow06, primarily requiring that the model be able to reproduce the $b_{\mathrm{J}}$ and $K$ luminosity functions at an acceptable level of accuracy, only examining the other data sets for the models which passed this test. Other published models have often included additional observational constraints from the start (e.g. Cole et al. 2000). Clearly, an alternative strategy to that followed here would be to emulate the full range of data at the outset, perhaps including both high- and low-redshift constraints. We have been initially cautious of this approach since the model implausibility must be combined across different data sets in a carefully thought-out manner and weighting the data from prior knowledge would require much consideration. Moreover, if the two data sets are in contradiction, this can lead to overconfident exclusion of parameter space regions, rather than pointing to a particular area in which the model needs improvement. The tension between the disc size and TF relation data sets is a clear case of this. Applying a strict requirement that the model match both data sets greatly restricts the acceptable parameter region. The physical cause of this tension is the baryonic contraction of the halo, something that is perennial problem in models of disc galaxies (cf. Cole et al. 2000). However, the degree of contraction is strongly dependent on the angular momentum distribution of the accreted material and the treatment of contraction in disc instabilities (see Benson \& Bower 2010). It is therefore questionable if we should reject models on the basis of this tension at the outset, and an approach of considering data sets separately may be preferable.

We find that the disc size, TF and gas mass to luminosity ratio data sets provide the strongest additional constraints (i.e. in addition to the luminosity function constraints). Qualitatively similar conclusions were reached previously by Cole et al. (2000), using an earlier version of the GALFORM model, and following the traditional approach of perturbing parameters away from their 'best-fitting' values and visually inspecting the results. In contrast, the methods we develop here are objective and do not rely on visual assessment. Although, data on colours and metallicity provide additional restrictions, at the level that is considered here, they provide little additional leverage over comparison of the luminosity functions. Future model changes that improve treatment of angular momentum and baryonic contraction should allow these data sets (and others 
that we have not considered here, such as the X-ray luminosities of groups and clusters; Bower et al. 2008) to play a more consistent role. Inclusion of high- $z$ data sets is also possible; however, there is clearly a balance to be struck between using all the available data to calibrate the model and holding some data sets in reserve to act as a test of the model's predictive (or rather 'post-dictive') power.

\section{CONCLUSIONS}

The GALFORM model is a semi-analytic model of the galaxy formation process that has been extensively used to understand the formation of galaxies. Different versions of GALFORM have used varying prescriptions for some of the key physical processes, and have been tuned to match different, though overlapping, observational data sets (Cole et al. 2000; Baugh et al. 2005; Bow06). In particular, the Bow06 version of the model, incorporating AGN feedback, has been successful in providing a good description of both the $b_{\mathrm{J}}$ - and $K$-band luminosity functions of present-day galaxies and the evolution of the $K$-band luminosity function with redshift. However, the Bow06 model represents one selection of parameters from a vast $16 \mathrm{D}$ parameter space (which is in turn a subspace of the full GALFORM parameter space), and it is natural to ask how unique the model is. In particular, we would like to identify the region of parameter space that is constrained in this way, allowing us to understand the degeneracies between the input parameters and their relative importance. Unfortunately, direct evaluation of the model with a uniform and sufficiently dense covering of the parameter space is not feasible because of the high-dimensionality of the parameter space and the relatively long run-time of the model. This is a common problem in many computer-modelling disciplines, such as climate change modelling, and there is great interest in developing efficient mathematical techniques that optimize the use of the available computing resource.

In this paper, we have used the model emulator technique (Craig et al. 1996, 1997; Kennedy \& O'Hagan 2001) to explore the full $16 \mathrm{D}$ parameter space of the Bow06 galaxy formation model. Rather than trying to evaluate the model directly at all points in parameter space, we run the model at a sparse sampling of points and then construct an emulator of the model that allows us to interpolate between these points. A key aspect of this construction is that we are not only able to establish an expectation value for the model's performance between runs, but are also able to encapsulate the degree of uncertainty in this estimate. Thus, we can use the emulator to identify regions in which the run outcome is uncertain and target additional evaluations there. By proceeding in waves of iterations, we focus the model evaluation down on a smaller and smaller region in which run outcomes are likely to accurately reproduce the observational data.

Another important aspect of our approach is that we introduce the concept of 'model discrepancy'. This is a small additional variance term that is included to explicitly account for the level of approximation inherent in the GALFORM model. This term means that model luminosity functions which lie within a factor of 1.54 of the observed luminosity functions are deemed acceptable, even if the observational errors are much smaller than this. This term ensures that we do not attempt to over fit the model and rule out regions of parameter space when this is not justified (given the approximate nature of the model).

The method is shown to be highly effective. We find that 0.014 per cent of the input parameter space produces model luminosity functions that are acceptable matches to the observed $b_{\mathrm{J}}$ - and $K$-band luminosity functions from Norberg et al. (2002) and Cole et al. (2001) (although in common with previous versions of the code, acceptable models tend to lie slightly above the faintest $K$-band measurements). However, we find that although the region of parameter space is small, acceptable fits can be obtained as parameters are adjusted over a large fraction of their input range. We show that the choice of parameters in the Bow06 model is not unique in reproducing the observational data and that other choices of parameters perform at least equally well: changes in one parameter may be compensated by adjusting several other parameters to leave the predicted luminosity functions almost unchanged. However, while some parameters play a vital role in adjusting the luminosity function to match the observational data, others have little effect on this (when adjusted within the range explored). Interestingly, these inactive parameters include the re-ionization parameters $\left(v_{\text {cut }}, z_{\text {cut }}\right)$, suggesting that these affect only galaxies below the faintest luminosities included in our luminosity function, and the merger parameters (these are masked by the dominance of disc instabilities in the model and, to some extent, the lack of morphological constraints).

In order to explore the parameter dependencies further, we have investigated using PCA to identify the optimal projections of the data. These allow us to identify the hyperplane on to which the data are constrained by the luminosity function data. This analysis reveals physically interesting interactions between the data, although each plane generally includes contributions from a large number of parameters. We have briefly explored how these directions can be rotated to reveal simple relations between the parameters that must be obeyed in order to obtain a well-fitting model. For example, we are able to quantify the relationship between the feedback parameters $\left(V_{\text {hot,disc }}, V_{\text {hot,burst }}, \alpha_{\text {hot }}\right)$, the return time-scale for reheated gas $\left(\alpha_{\text {reheat }}\right)$ and the star formation parameter $\left(\epsilon_{\star}^{-1}\right)$. However, although these relations can provide a useful check on physical intuition and understanding, they do not reproduce the full non-linear complexity of the model that is captured by the model emulator.

We have also briefly explored the impact of adding additional data sets to constrain the model by evaluating a simple $\chi^{2}$ statistic against observational data sets describing disc sizes, the TF relation, gas metallicity, the gas-mass-to-light ratio, the galaxy colour distribution and the black hole mass-bulge mass correlation. We find that the disc sizes, the gas-to-light ratio and the TF relation place the strongest additional constraints on the model. Similar conclusions were reached by Cole et al. (2000) using an earlier version of GALFORM, who followed the traditional approach of manually varying parameters and then visually inspected the results. The great advantage of the new approach presented here is that it is automated, objective and reveals the couplings between different parameters in their effects on observable quantities. However, we find that the direction of constraint from the disc size data is in contradiction to the constraints imposed by the gas mass to luminosity ratio and TF relation data. The disc size data present a particular challenge for the Bow06 model, and the issue has been explored in detail in González et al. (2009).

The model considered here was deliberately restricted to the version of GALFORM used in Bow06. In future papers we will apply the same general methods to a broader class of GALFORM models, including processes such as feedback from galaxy superwinds (Benson et al. 2003a), variations in the IMF (Baugh et al. 2005), ram-pressure stripping (Font et al. 2008), and X-ray emission from hydrostatic haloes (Bower et al. 2008), greatly increasing the model parameter space. This leads to a new challenge - but it is one that we now have the statistical techniques to meet. 


\section{ACKNOWLEDGMENTS}

RGB acknowledges the support of a Durham-University Christopherson-Knott Fellowship. IRV and MG acknowledge the support of the Basic Technology initiative as part of the Managing Uncertainty for Complex Models project. IRV acknowledges the support of an EPSRC mobility fellowship. AJB acknowledges the support of the Gordon \& Betty Moore Foundation. CGL acknowledges support from the STFC rolling grant to the ICC.

\section{REFERENCES}

Baugh C. M., Lacey C. G., Frenk C. S., Granato G. L., Silva L., Bressan A., Benson A. J., Cole S., 2005, MNRAS, 356, 1191

Benson A. J., Bower R. G., 2010, MNRAS, 405, 1573

Benson A. J., Bower R. G., Frenk C. S., Lacey C. G., Baugh C. M., Cole S., 2003a, ApJ, 599, 38

Benson A. J., Frenk C. S., Baugh C. M., Cole S., Lacey C. G., 2003b, MNRAS, 343, 679

Bower R. G., Benson A. J., Malbon R., Helly J. C., Frenk C. S., Baugh C. M., Cole S., Lacey C. G., 2006, MNRAS, 370, 645 (Bow06)

Bower R. G., McCarthy I. G., Benson A. J., 2008, MNRAS, 390, 1399

Cattaneo A., Dekel A., Devriendt J., Guiderdoni B., Blaizot J., 2006, MNRAS, 370, 1651

Cole S., Aragon-Salamanca A., Frenk C. S., Navarro J. F., Zepf S. E., 1994, MNRAS, 271, 781

Cole S., Lacey C. G., Baugh C. M., Frenk C. S., 2000, MNRAS, 319, 168

Cole S. et al., 2001, MNRAS, 326, 255

Craig P. S., Goldstein M., Seheult A. H., Smith J. A., 1996, in Bernardo J. M., Berger J. O., Dawid A. P., Smith A. F. M., eds, Bayesian Statistics 5. Clarendon Press, Oxford, p. 69

Craig P. S., Goldstein M., Seheult A. H., Smith J. A., 1997, in Gatsonis C., Hodges J. S., Kass R. E., McCulloch R., Rossi P., Singpurwalla N. D., eds, Case Studies in Bayesian Statistics, Vol. 3. Springer-Verlag, New York, p. 36

Craig P. S., Goldstein M., Rougier J. C., Seheult A. H., 2001, J. Am. Stat. Assoc., 96, 717

Crain R. A. et al., 2009, MNRAS, 399, 1773

Cressie N., 1991, Statistics for Spatial Data. Wiley, Chichester

Croton D. J. et al., 2006, MNRAS, 367, 864

Currin C., Mitchell T., Morris M., Ylvisaker D., 1991, J. Am. Stat. Assoc., 86, 953

De Finetti B., 1974, Theory of Probability. Wiley, London

de Jong R. S., Lacey C., 2000, ApJ, 545, 781

De Lucia G., Blaizot J., 2007, MNRAS, 375, 2

De Lucia G., Kauffmann G., White S. D. M., 2004, MNRAS, 349, 1101

Dunkley J. et al., 2009, ApJS, 180, 306

Font A. S. et al., 2008, MNRAS, 389, 1619

Gnedin N. Y., Tassis K., Kravtsov A. V., 2009, ApJ, 697, 55

Goldstein M., Rougier J. C., 2009, J. Stat. Planning Inference, 139, 1221

Goldstein M., Wooff D. A., 2007, Bayes Linear Statistics: Theory and Methods. Wiley, Chichester

González J. E., Lacey C. G., Baugh C. M., Frenk C. S., Benson A. J., 2009, MNRAS, 397, 1254

Gonzalez-Perez V., Baugh C. M., Lacey C. G., Almeida C., 2009, MNRAS, 398, 497

Granato G. L., Lacey C. G., Silva L., Bressan A., Baugh C. M., Cole S., Frenk C. S., 2000, ApJ, 542, 710
Granato G. L., De Zotti G., Silva L., Bressan A., Danese L., 2004, ApJ, 600, 580

Häring N., Rix H.-W., 2004, ApJ, 604, L89

Heitmann K., Higdon D., White M., Habib S., Williams B. J., Wagner C., 2009, ApJ, 705, 156

Henriques B. M. B., Thomas P. A., Oliver S., Roseboom I., 2009, MNRAS, 396,535

Higdon D., Kennedy M., Cavendish J. C., Cafeo J. A., Ryne R. D., 2004, SIAM J. Sci. Comput., 26, 448

Huchtmeier W. K., Richter O.-G., 1988, A\&A, 203, 237

Jolliffe I. T., 2002, Principal Component Analysis. Springer Series in Statistics. Springer, New York

Kampakoglou M., Trotta R., Silk J., 2008, MNRAS, 384, 1414

Kang X., Jing Y. P., Silk J., 2006, ApJ, 648, 820

Kauffmann G., White S. D. M., Guiderdoni B., 1993, MNRAS, 264, 201

Kauffmann G., Colberg J. M., Diaferio A., White S. D. M., 1999, MNRAS, 307, 529

Kennedy M. C., O'Hagan A., 2001, J. R. Stat. Soc. Ser. B., 63, 425

Kennicutt R. C., 1983, ApJ, 272, 54

Kim H.-S., Baugh C. M., Cole S., Frenk C. S., Benson A. J., 2009, MNRAS, 400, 1527

Lacey C., Silk J., 1991, ApJ, 381, 14

Malbon R. K., Baugh C. M., Frenk C. S., Lacey C. G., 2007, MNRAS, 382, 1394

Menci N., Fontana A., Giallongo E., Salimbeni S., 2005, ApJ, 632, 49

Menci N., Fontana A., Giallongo E., Grazian A., Salimbeni S., 2006, ApJ, 647, 753

Monaco P., Fontanot F., Taffoni G., 2007, MNRAS, 375, 1189

Nagashima M., Totani T., Gouda N., Yoshii Y., 2001, ApJ, 557, 505

Norberg P. et al., 2002, MNRAS, 336, 907

Parkinson H., Cole S., Helly J., 2008, MNRAS, 383, 557

Parry O. H., Eke V. R., Frenk C. S., 2009, MNRAS, 396, 1972

Oakley J., O'Hagan A., 2002, Biometrika, 89, 769

Oakley J., O’Hagan A., 2004, J. R. Stat. Soc. B., 66, 751

O’Hagan A., 2006, Reliability Eng. System Safety, 91, 1290

Pizagno J. et al., 2007, AJ, 134, 945

Santner T. J., Williams B. J., Notz W. I., 2003, The Design and Analysis of Computer Experiments. Springer-Verlag, New York

Schaye J. et al., 2010, MNRAS, 402, 1536

Schneider M. D., Knox L., Habib S., Heitmann K., Higdon D., Nakhleh C., 2008, Phys. Rev. D, 78, 6

Somerville R. S., Primack J. R., 1999, MNRAS, 310, 1087

Somerville R. S., Hopkins P. F., Cox T. J., Robertson B. E., Hernquist L., 2008, MNRAS, 391, 481

Springel V. et al., 2005, Nat, 435, 629

Stein M., 1987, Technometrics, 29, 143151

Tremonti C. A. et al., 2004, ApJ, 613, 898

Trotta R., 2008, Contempoary Phys., 49, 71

Vernon I., Goldstein M., Bower R. G., 2010, Case Studies in Baysean Statistics. MUCM Technical Report 10-03 2.1.8 IV, http://mucm.group.shef.ac.uk, submitted

Weinmann S. M., van den Bosch F. C., Yang X., Mo H. J., 2006, MNRAS, 366,2

White S. D. M., Frenk C. S., 1991, ApJ, 379, 52

Williams C. K. I., 2002, in Arbib M. A., ed., Handbook of Brain Theory and Neural Networks. MIT Press, Cambridge, MA

Zito T., Wilbert N., Wiskott L., Berkes P., 2009, Front. Neuroinform., 2, 8

This paper has been typeset from a $\mathrm{T}_{\mathrm{E}} \mathrm{X} / \mathrm{L} \mathrm{T} \mathrm{E} \mathrm{X}$ file prepared by the author 